ERNEST DRLANDD LAWRENCE

BERKELEY NATIONAL LABDRATURY

An Investigation of Polarized Atomic Photofragments Using the Ion Imaging Technique

Allan S. Bracker

Chemical Sciences Division

December 1997

Ph.D. Thesis
RECEIVED

MAY O 81998

OSTI

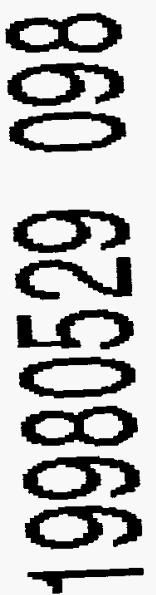




\section{DISCLAIMER}

This document was prepared as an account of work sponsored by the United States Government. While this document is believed to contain correct information, neither the United States Government nor any agency thereof, nor The Regents of the University of California, nor any of their employees, makes any warranty, express or implied, or assumes any legal responsibility for the accuracy, completeness, or usefulness of any information, apparatus, product, or process disclosed, or represents that its use would not infringe privately owned rights. Reference herein to any specific commercial product, process, or service by its trade name, trademark, manufacturer, or otherwise, does not necessarily constitute or imply its endorsement, recommendation, or favoring by the United States Government or any agency thereof, or The Regents of the University of California. The views and opinions of authors expressed herein do not necessarily state or reflect those of the United States Government or any agency thereof, or The Regents of the University of California.

Ernest Orlando Lawrence Berkeley National Laboratory is an equal opportunity employer. 


\title{
An Investigation of Polarized Atomic Photofragments Using the Ion Imaging Technique
}

\author{
Allan Stephen Bracker \\ Ph.D. Thesis \\ Department of Chemistry \\ University of California, Berkeley \\ and \\ Chemical Sciences Division \\ Ernest Orlando Lawrence Berkeley National Laboratory \\ University of California \\ Berkeley, CA 94720
}

December 1997

\section{MASTER}

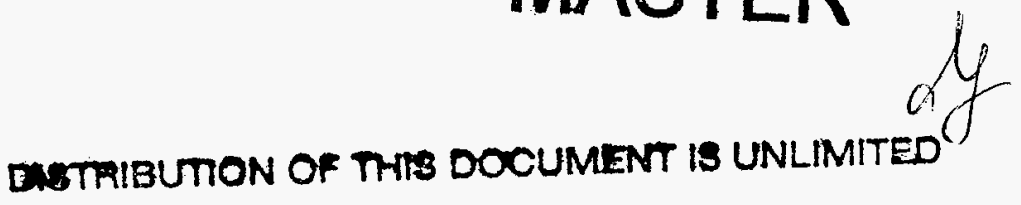

This work was supported by the Director, Office of Energy Research, Office of Basic Energy Sciences, Chemical Sciences Division, of the U.S. Department of Energy under Contract No. DE-AC03-76SF00098. 


\section{An Investigation of Polarized Atomic Photofragments \\ Using the Ion Imaging Technique}

by

Allan Stephen Bracker

B. S. (Stanford University) 1989

A dissertation submitted in partial satisfaction of the requirements for the degree of

Doctor of Philosophy

in

Chemistry

in the

GRADUATE DIVISION

of the

UNIVERSITY of CALIFORNIA at BERKELEY

Committee in charge:

Professor Yuan T. Lee, Chair

Professor Daniel M. Neumark

Professor Raymond Chiao

Fall 1997 


\section{An Investigation of Polarized Atomic Photofragments Using the Ion Imaging Technique}

Copyright $@ 1997$

by

Allan S. Bracker

The Government reserves for itself and others acting on its behalf a royalty free, nonexclusive, irrevocable, world-wide license for goverrmental purposes to publish, distribute, translate, cuplicate, exhibit, and perform any such data copyrighted by the contractor.

The U.S. Department of Energy has the right to use this document for any purpose whatsoever including the right to reproduce all or any part thereof 


\title{
Abstract \\ An Investigation of Polarized Atomic \\ Photofragments using the Ion Imaging Technique
}

\author{
by \\ Allan Stephen Bracker \\ Doctor of Philosophy in Chemistry \\ University of California at Berkeley \\ Professor Yuan T. Lee, Chair
}

This thesis describes measurement and analysis of the recoil angle dependence of atomic photofragment polarization (atomic $\mathbf{v}-\mathbf{J}$ correlation). This property provides information on the electronic rearrangement which occurs during molecular photodissociation.

Chapter 1 introduces concepts of photofragment vector correlations and reviews experimental and theoretical progress in this area. The correlations of interest include the velocity anisotropy, angular momentum polarization, and the more detailed $\mathbf{v}-\mathbf{J}$ correlation. Special attention is given to polarization of atomic angular momentum.

Chapter 2 describes the photofragment ion imaging technique, which we have used to study the atomic $\mathbf{v}-\mathbf{J}$ correlation in chlorine and ozone dissociation. With this technique, a three-dimensional velocity vector distribution of state-selectively ionized photofragments is mapped into a two-dimensional spatial distribution. Photofragments are ionized with a resonant multiphoton technique, which also provides sensitivity to angular momentum polarization. This polarization is observed as small modulations in the ion images, which depend on the probe laser polarization and on the symmetry of the chosen spectroscopic probe transition. 
Chapter 3 outlines a method for isolating and describing the contribution to the image signal which is due exclusively to angular momentum alignment. This method is based on recently-developed theoretical tools in combination with wellknown two-photon linestrength expressions. We show that four molecular excitation processes-parallel and perpendicular incoherent excitation and two types of coherent excitation-each contribute a unique spatial structure to the ion images. These mechanisms are represented by a set of alignment anisotropy parameters, which are analogous to the familiar velocity anisotropy parameter $\beta$.

Ion imaging results are presented and discussed in Chapter 4 . We show that the alignment contribution to images of ground state chlorine atom photofragments results primarily from a perpendicular optical excitation in the parent $\mathrm{Cl}_{2}$ molecule. In order to adequately simulate the data, it was necessary to include contributions from both incoherent and coherent excitation. We also argue that nonadiabatic transitions must have occurred in the asymptotic region of the molecular potential energy curves. Preliminary measurements of polarized $\mathrm{O}\left({ }^{1} \mathrm{D}_{2}\right)$ from ozone dissociation show a strong polarization effect, which depends on the vibrational state of the $\mathrm{O}_{2}$ partner fragment.

Chapter 5 discusses a different set of experiments on the three-fragment dissociation of azomethane. We measured appearance times and kinetic energy release for two vibrational quantum states of the methyl radical photofragments and have characterized multiphoton processes which would otherwise complicate the analysis of these data. The results are consistent with a previously proposed mechanism of a concerted dissociation following randomization of internal energy. 
To my family 


\section{Contents}

Table of Contents $\quad v$

List of Figures vii

1 Introduction 1

1.1 Perspective ...................... 1

1.2 Vector Correlations in Photodissociation . . . . . . . . 4

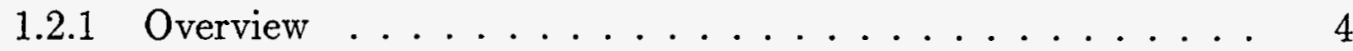

1.2 .2 Velocity Anisotropy . . . . . . . . . . . . . . . . . 4

1.2.3 Angular Momentum Polarization . . . . . . . . . 7

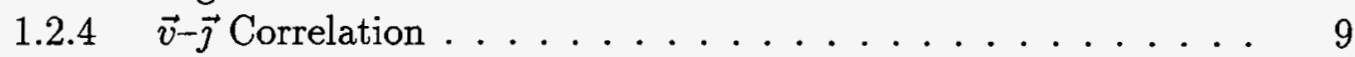

1.3 Atomic Angular Momentum Polarization . . . . . . . . . . . . . 12

2 Experimental Methods and Principles 19

2.1 Overview . . . . . . . . . . . . . . . . . . . . 19

2.2 State-selective Spectroscopic Detection with REMPI . . . . . . . 22

2.2.1 General Considerations and Applications of REMPI . . . . . . 22

2.2 .2 Producing and Using Ultraviolet Laser Light . . . . . . . . . 27

2.3 Ion Imaging and Time-of-Flight Mass Spectrometry . . . . . . . . . 32

2.3.1 Operating Principle and General Design Considerations . . . . 32

2.3.2 Extraction Optics and Position-Sensitive Detector . . . . . . 35

2.3.3 Treatment of Raw Image Data . . . . . . . . . . . . . . 37

$2.4 \vec{v}-\vec{\jmath}$ Correlation Measurements . . . . . . . . . . . . . . 39

3 Data Analysis Method 45

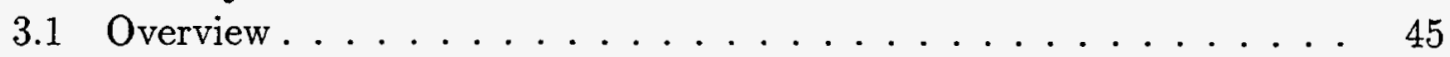

3.2 State Multipoles. . . . . . . . . . . . . . . . . 46

3.3 Polarization Anisotropy Parameters and Specific Expressions for

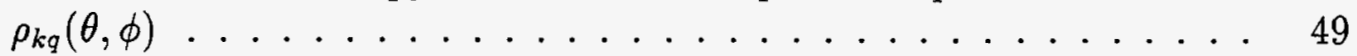

3.4 Two-photon Transition Probabilities . . . . . . . . . 54

3.5 Alignment Image Basis Functions . . . . . . . . . . . . . . . . 59 
vi

4 Results and Discussion $\quad 65$

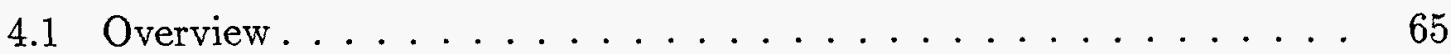

4.2 Introduction: Chlorine Photodissociation . . . . . . . . . 66

4.3 Experimental Results on Chlorine Dissociation at $355 \mathrm{~nm}$. . . . . 72

4.3 .1 Chlorine Ion Image Data . . . . . . . . . . . . . . . 72

4.3.2 Total Alignment Measurement . . . . . . . . . . 72

4.3 .3 Alignment Images . . . . . . . . . . . . . . . . . . . . 78

4.4 A Closer Look at the Dynamical Functions: Implications for Chlorine Dissociation . . . . . . . . . . . . . 82

4.5 Polarized $\mathrm{O}\left({ }^{1} D_{2}\right)$ from the Dissociation of Ozone at $266 \mathrm{~nm} \ldots \ldots 8$

5 Photochemistry of Azomethane Following Absorption of $\begin{array}{ll}\text { One or More Photons } & 97\end{array}$

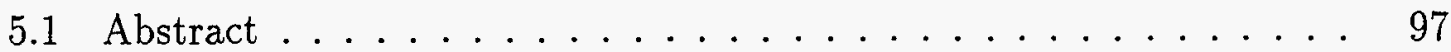

5.2 Introduction and Background . . . . . . . . . . . . . . 98

5.3 Experimental Methods . . . . . . . . . . . . . . . . 104

5.4 Results and Discussion . . . . . . . . . . . . . . . . 108

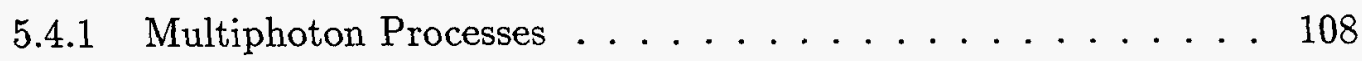

5.4 .2 Ultraviolet Dissociation . . . . . . . . . . . . . 111

5.5 Conclusion . . . . . . . . . . . . . . . 130

$\begin{array}{ll}\text { References } & 131\end{array}$ 


\section{List of Figures}

1.1 Vectors in photodissociation dynamics ............ 5

1.2 Distributions which describe vector correlations . . . . . . . . 6

1.3 Oriented and aligned ensembles ............... 8

1.4 Mocular orbital view of atomic photofragment polarization . . . . . 13

1.5 Factors affecting polarization of atomic photofragments . . . . . . . 14

2.1 Schematic of ion imaging experiment . . . . . . . . . . . . . 21

2.2 Schematic of experimental apparatus . . . . . . . . . . 23

2.3 Relationship of transition moment to angular momentum vector . . . 25

2.4 REMPI schemes for chlorine and oxygen atoms . . . . . . . . . 29

2.5 Saturation behavior of both steps in chlorine 2+1 REMPI . . . . . 31

2.6 Operating principle of Wiley-McClaren 'space focussing' condition . . 34

2.7 Ion extraction optics design . . . . . . . . . . . . . 36

2.8 Symmetrization of raw data images . . . . . . . . . . . . . . . . 38

2.9 Standard deviation associated with symmetrization . . . . . . . . 39

2.10 Schematic of $\mathbf{v}-\mathbf{J}$ correlation measurement principle . . . . . . . 41

2.11 Experimental geometries for the study of $\mathbf{v}-\mathbf{J}$ correlations . . . . . 42

3.1 Classical $P(\mathbf{J})$ from coherent and incoherent excitations . . . . . . . 53

3.2 Clarification of variables for $3 \mathrm{D}$ to $2 \mathrm{D}$ transformation . . . . . . . 61

3.3 (color) Alignment image basis functions . . . . . . . . . . . . 63

4.1 Absorption spectrum and potential energy curves for chlorine . . . . 67

4.2 State correlation diagram for chlorine . . . . . . . . . . 69

4.3 (color) Ion image data and difference images. $\left({ }^{2} D_{3 / 2}^{\circ}\right.$ state probe $)$. 74

4.4 (color) Ion image data and difference images. $\left({ }^{2} S_{1 / 2}^{o}\right.$ state probe) . . . 75

4.5 Total alignment measurement .................... 76

4.6 Depolarization due to hyperfine interaction . . . . . . . . . . 77

4.7 Geometry I data with standard deviation. Contour plots . . . . . . . 79

4.8 Geometry II data with standard deviation. Contour plots . . . . . . . 80

4.9 (color) Simulation of alignment images . . . . . . . . . . . . 81

4.10 Nonadiabatic transitions required for $\mathrm{Cl}$ alignment . . . . . . . . . 87 
viii

4.11 Ozone absorption spectrum and potential energy surfaces . . . . . . 90

4.12 Ion image data for ozone dissociation at $266 \mathrm{~nm} \ldots$. . . . . . . . . . . . . 92

4.13 Radial distribution of signal in ozone images . . . . . . . . . . . . . . 93

4.14 Angular distribution of signal in ozone images . . . . . . . . . . 95

5.1 Azomethane absorption spectrum and potential energy curves . . . 99

5.2 Synchronous, concerted, and stepwise trajectories . . . . . . . . . 102

5.3 Dissociation mechanism from $a b$ initio study . . . . . . . . . . . . . 103

5.4 Principle of core-sampling TOFMS technique . . . . . . . . . . . . 107

5.5 Comparison of mass spectra at $330 \mathrm{~nm}$ and $355 \mathrm{~nm}$. . . . . . . . 110

5.6 Azomethane ionization channels . . . . . . . . . . . . . 112

5.7 VUV dissociative ionization of azomethane . . . . . . . . . . . 113

5.8 Dependence of $\mathrm{CH}_{3}$ REMPI signal on laser time delay . . . . . . . . . 115

5.9 Subtraction of non-resonant signal from REMPI signal . . . . . . . 116

5.10 TOFMS profiles: $355 \mathrm{~nm}$ dissociation . . . . . . . . . . . . . . . 117

5.11 TOFMS profiles: $330 \mathrm{~nm}$ dissociation . . . . . . . . . . . . . 118

5.12 Simulating core-sampled TOFMS profiles . . . . . . . . . . . . 120

5.13 Notation for voltages and dimensions of mass spectrometer . . . . . . 122

5.14 Translational energy distributions: $355 \mathrm{~nm}$ dissociation . . . . . . . 124

5.15 Translational energy distributions: $330 \mathrm{~nm}$ dissociation . . . . . . . 125

5.16 Comparison with results of North et al. . . . . . . . . 126 


\section{Acknowledgements}

During my stay at Berkeley, I have had the opportunity to work, interact and learn from a wide variety of people. My foremost thanks go to my research advisor Yuan Lee. Yuan's group provides an unsurpassed opportunity to do great research-a consequence of the great depth of intellectual and physical resources in the group and the confidence which Yuan has in the abilities of his graduate students. The freedom that we are given to choose the direction of our thesis research is undoubtedly a double-edged sword, since in the end, we must answer for the success or failure of our decisions. For me, this additional risk has been of great benefit, as Yuan confidently encouraged me to pursue a fascinating thesis topic which was well off the beaten path for his group.

Since Yuan left for Taiwan in 1994, Arthur Suits has handled the day-to-day operations of the group. In the last few years, Arthur has managed to build up his own group at LBL and prevent serious fiscal hemmorhaging, even as most of Yuan's laboratory hardware was being shipped off to various parts of the world. During my fourth year, it was Arthur who first suggested studying photofragment orbital alignment, and in the three years since, this topic has developed into a fruitful thesis project.

The success of the orbital alignment campaign owes a great deal to Professor Oleg Vasyutinskii from the Ioffe Institute in St. Petersburg, Russia. Our very enjoyable collaboration during 1996-7, in both St. Petersburg and Berkeley, was for me by far the most concentrated and productive period of learning on the subject of photofragment polarization. Oleg played the critical role in the theoretical analysis of our ion image data and in defining future directions for experiments. I hope that the collaboration between Oleg and Arthur will continue long after I leave Berkeley.

My closest and most prolonged interactions at Berkeley were with two fellow graduate students, who are both excellent scientists and good friends-Simon North and David Blank. At two different stages of the azomethane project, I had the chance to work briefly in the lab with both Simon and Dave, and I only regret that I didn't have even more extended collaborations with both of them. Simon and I started 
graduate school the same year, and to this day, we continue to learn from each other. I look forward to visiting Simon and his wife Diana at their new home in the great foreign land of Texas. For the last couple of years, Dave and I have enjoyed keeping the torch burning during the final chapter of the Lee Group at Berkeley.

The process of learning about a new theoretical topic is always more efficient when there are others around to discuss with. Working with Oleg was critical in this regard, and extended conversations with others have been extremely valuable as well. Simon and I were learning the basics of angular momentum polarization at about the same time (while he was at Brookhaven), and we tackled common conceptual blocks over the phone or during occasional visits. I also benefitted greatly from several extended conversations with Greg Hall, especially during my 1995 visit to Brookhaven. In the latter stages of the chlorine study, Peter Rakitzis stimulated several thought-provoking discussions, particularly in our daylong meeting together with Oleg.

When I started graduate school, my laboratory collaborator and mentor was Marc Vrakking. Working with Marc on the $\mathrm{D}+\mathrm{H}_{2}$ project in DG30 was an excellent jump start to the rigors and challenges of graduate school, and I could hardly have asked to learn from a more qualified experimentalist. During my second year, I also enjoyed the additional company of Toshi Suzuki, who has since gone on to found a groundbreaking imaging effort of his own. After a long hiatus spent in the D71 laboratory, I returned to DG30 in my fifth year, where I finished up the chlorine measurements and explored some other halogenated compounds in collaboration with Holly Bevsek and Cortney Sailes, before passing the machine along to them for their $\mathrm{O}_{4}$ venture.

During my forays to the east end of D-level, I worked with several people. Toni Soliva and I put the finishing touches on the new machine in D71, and Jinchun Xie joined us with Andy Kung's new IR laser for some initial experiments on ammonia. I enjoyed hearing stories from Jinchun about the other great chemical dynamics dynasty on the West Coast-the Zare Group. After finishing my much-prolonged azomethane experiments, I passed the machine on to Dave Blunt, but in mid-1996, joined him again briefly for a look at ozone. I made one final appearance in D71 
during 1997, when Oleg and I muscled in on Eloy Wouters to do some calibrations for the chlorine alignment analysis. I would have liked to work longer with Eloy and have enjoyed poking my nose into his experiments as a break from writing my thesis. Eloy also deserves many thanks for my smooth transition into $\mathrm{LTT}_{\mathrm{E}} \mathrm{X}$.

Those of us in the last wave or two of Lee group graduations pondered the end of an era as we pushed each other along. Hongtao Hou and Doo Wan Boo, who started graduate school just one year before me, were good friends who set examples for my own impending GRC/prelim/thesis/jobhunt trials. For many years, I enjoyed extended conversations with officemates Laura Smoliar and James Chesko. After being out of touch for a year or two, they are both back in the Bay Area and we are once again discussing life's trials and rewards. With the last set of graduationsincluding T.T. Miau, Cheryl Longfellow, Cindy Berry, Dave Blank, and myself, the Berkeley Lee group as we know it seems to end.

Many people have contributed to the critical ancillary tasks which allowed our research group to function. System administrators Zhifeng Liu, Dave Blunt and Eloy Wouters kept leea running smoothly even in the face of reset-button-pressing maniacs and repeated power failures. In the pre-Linux days, Jim Meyers and James Chesko played a similar role. Anne Lawhead and Marian Grebanier were our interface to the outside world, and their importance is all the more clear now that that office is unstaffed. The various College of Chemistry shops constructed and repaired much of the custom hardware used in our experiments. In the machine shop, I especially appreciated the chance to discuss Lee group goings on (and sometimes College politics) with Hans Graetsch.

I enjoyed outstanding research experiences even before graduate school. At Stanford, Steve George and his students provided excellent academic advice and an enjoyable opportunity to do surface science. In 1990, with help from Dick Zare, I was able to spend seven months at the Max-Planck-Institute for Quantum Optics in Karl Kompa's section. There, Jochen Wanner introduced me to Chemical Reaction Dynamics and steered me in the direction of Yuan's group. In that relatively short time, I learned much about science and about German language and culture. My stay in Germany, and particularly the collaboration with Jochen, is one of my most 
xii

memorable experiences.

The most important and influential people in my life are my family-my parents and my brother Kiko. Their love and encouragement over many years has been an inspiration to me. I dedicate this thesis to them.

The research described in this thesis was supported by the Director, Office of Energy Research, Office of Basic Energy Sciences, Chemical Sciences Division, of the United States Department of Energy. In addition, the collaboration with Professor Vasyutinskii was supported by a Cooperative Grant from the Civilian Research and Development Foundation. From 1991-93, I was supported by a Predoctoral Fellowship from the National Science Foundation. 


\section{Chapter 1}

\section{Introduction}

\section{$1.1 \quad$ Perspective}

Scientists are accustomed to describing the potential benefits and broader implications of their work to funding agencies, friends, and interested citizens. Presumably this task is straightforward for engineers and applied scientists, whose work is closely related to immediate practical benefits. For those engaged in basic research, it can be more difficult. Most Americans respect the search for knowledge and are largely supportive of basic science, but their support has practical rather than academic motivations. Indeed, the modern research establishment is founded on the expectation that basic science will yield useful results. This view was stated by Vannevar Bush in 'Science: The Endless Frontier', a 1945 report to President Franklin Roosevelt:

"On the wisdom with which we bring science to bear in the war against disease, in the creation of new industries, and in the strengthening of our Armed Forces depends in large measure our future as a nation." [1]

For some types of basic research, especially in the fundamental physical sciences, it is often not obvious what practical benefits, if any, will result. But with sufficient foresight and creativity, scientists in many fields can imagine some range of useful products, processes or concepts stemming from their work, even if in the distant future. The field of chemical reaction dynamics, for example, has important practical 
consequences for areas such as atmospheric and combustion chemistry. Researchers often appeal to the search for more efficient fuels and cleaner air, which the public easily appreciates.

But the reality is that many of the benefits of basic research are rather esoteric and not readily understandable or appealing to policymakers or the public. For those of us studying chemical reaction dynamics, it may be possible to describe the combustion or atmospheric relevance of individual research projects, but in many instances this no longer accurately reflects our own motivations or the primary relevance of the research. A more appropriate and honest view is that the major purpose of basic research is not to generate practical applications for immediate use by society, but instead to provide the intellectual groundwork for further progress in many areas of science. According to this view, the unexplained phenomena of the past, which were formerly objects of study in their own right, eventually join a powerful array of concepts that serve as the modern intellectual artillery for further research. This philosophy was not lost on Bush, who was himself an engineer ${ }^{1}$ :

"As long as [the centers for research] are vigorous and healthy and their scientists are free to pursue the truth wherever it may lead, there will be a flow of new scientific knowledge to those who can apply it to practical problems in Government, in industry, or elsewhere" [1]

Our understanding of the genetic code, which translates RNA sequences into proteins, is an intellectual triumph of basic research in the 1960s. Today, this critical intellectual and practical tool must be understood at some level by anyone involved in fundamental or applied biological science. In many physical sciences, the wave nature of matter is a recurring theme with widespread consequences. These well-known examples are familiar even to non-scientists, but a much larger set of less familiar concepts plays a similar role within narrower subdisciplines. These more modest discoveries are among the major products of basic research which unfortunately are known only to the specialists.

\footnotetext{
${ }^{1}$ Bush's statement should not be interpreted too narrowly. Bush was a believer in the so-called 'linear model of innovation', where basic science leads to technology in a one-way flow of knowledge. With the benefit of 50 years of hindsight, it has become clear that more complex feedback processes occur.
} 
In the field of chemical reaction dynamics, we are concerned with the microscopic forces and motions which are at play during chemical processes-from reactants to transition state to products. The motions (or quantum states) of interest include vibration, rotation, translation, electronic orbital and spin states. In a broad sense, we can speak of two types of measurable quantities-scalar and vector properties. Scalar properties consist primarily of the energies and populations of the various quantum states, while vector properties are concerned with the directions and anisotropies of motion. The study of these anisotropies and asymmetries in chemical reactions is sometimes referred to as dynamical stereochemistry [2-4]. For more than twenty years, two such properties have been a crucial part of the standard repertoire of conceptual tools for both experimentalists and theorists. The more familiar of the two is the velocity anisotropy, which serves as a useful indicator of excited state symmetries and lifetimes. The second is angular momentum polarization (orientation and alignment), which provides information on the torques acting during collision or dissociation.

The $\mathbf{v}$-J correlation is a more detailed vector property which describes the recoil angle dependence of the angular momentum polarization. Within the last decade, this property has become experimentally and theoretically accessible to a wide range of investigators. For the case of diatomic photofragments, the v-J correlation has joined the ranks of commonly-measured quantities which serve as useful diagnostics for unraveling photodissociation dynamics. This dissertation is concerned with a less-familiar type of $\mathbf{v}-\mathbf{J}$ correlation involving the electronic angular momentum of atomic photofragments. In more familiar language, this property corresponds to the alignment of the electronic orbitals of an atom produced in the dissociation of a molecule. Measurements of this property provide information on the electronic rearrangements which occur during photodissociation. The field of atomic photofragment polarization has been studied by a small group of specialists since the early 1980 s, but only in the past few years has it gained the attention of the wider chemical reaction dynamics community, largely through reports of the atomic $\mathbf{v}-\mathbf{J}$ correlation. It is likely that the demonstration phase of this observable will soon be over, and that it will reach the threshold to become a convenient tool for investigating molecular photofragmentation dynamics. 


\subsection{Vector Correlations in Photodissociation}

\subsubsection{Overview}

The vectors with which we are concerned are summarized in Fig 1.1. The polarization vector $\mathbf{E}$ of linearly polarized dissociation light is frequently chosen as the laboratory-fixed $Z$-axis. For circularly polarized or unpolarized light, the propagation direction $\mathbf{k}$ is chosen. When a beam of light interacts with an isotropic gas sample, the polarization anisotropy of the light will be transferred to the gas molecules via their transition moments $\boldsymbol{\mu}$, with the shape of the excited transition moment distribution determined by the excitation probability $|\boldsymbol{\mu} \cdot \mathbf{E}|^{2}=\cos ^{2} \theta_{\mu}$. A portion of this anisotropy is subsequently reflected in the laboratory-frame distributions of the photofragment recoil velocity vectors $\mathbf{v}$ and angular momentum vectors $\mathbf{J}$. The $\mathbf{v}$ and $\mathbf{J}$ anisotropies are strongest when the dissociation occurs rapidly and are attenuated by parent rotation, although some portion of each will survive as a consequence of total angular momentum conservation. Finally, and of greatest relevance for this work, there usually exists a correlation between $\mathbf{v}$ and $\mathbf{J}$. These three vector correlations are summarized schematically in Fig 1.2 and are described in greater detail in the following sections.

\subsubsection{Velocity Anisotropy}

The photofragment angular distribution or velocity anisotropy (E-v correlation) is the most frequently measured vector correlation, since it depends in a straightforward way on properties which are of fundamental interest in photodissociation dynamics-the excited state symmetry and lifetime. The first laboratory observation of this effect was reported in 1967 by Solomon [5], who directly mapped the angular distribution onto the inner surface of a glass bulb. The first theoretical description of the velocity anisotropy was provided by Zare in the $1960 \mathrm{~s}[6,7]$. The widely-used result of this treatment, which can be derived using either classical or quantum-mechanical methods, is an expression for the photofragment density as a 

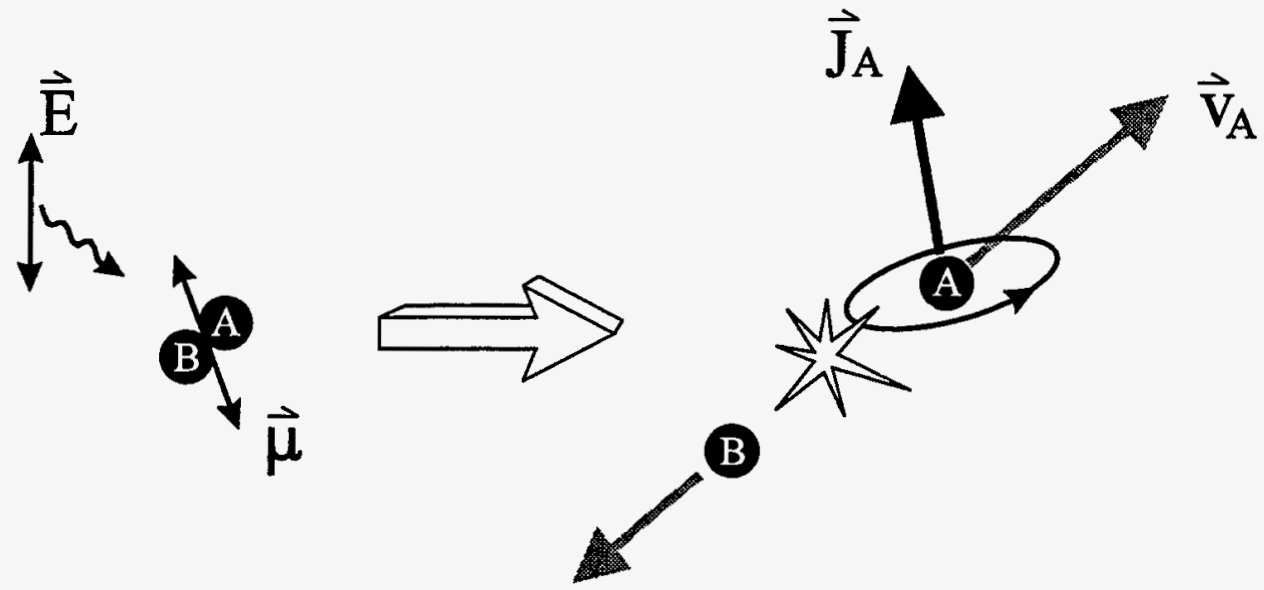

Figure 1.1: Vectors in photodissociation dynamics. The photon, described by propagation vector $\mathbf{k}$ and polarization $\mathbf{E}$, interacts with the molecule through its transition moment $\boldsymbol{\mu}$. After dissociation into fragments $\mathrm{A}$ and $\mathrm{B}$, the distributions of the velocity vector $\mathbf{v}$ and angular momentum vector $\mathbf{J}$ are generally polarized in space and with respect to each other.

function of recoil angle:

$$
P\left(\theta_{v}\right) \propto 1+\beta P_{2}\left(\cos \theta_{v}\right),
$$

where the angle $\theta_{v}$ is measured between the photolysis polarization vector $\mathbf{E}$ and the photofragment recoil vector $\mathbf{v}$.

The anisotropy parameter $\beta$ is defined as $\left\langle P_{2}(\cos \chi)\right\rangle$, where $\chi$ is the angle between the molecular transition moment and the asymptotic fragment recoil velocity vector, and the ensemble average is over all dissociating parent molecules. Parameter $\beta$ ranges from -1 to 2 for linearly polarized light along the $Z$-axis. For diatomic molecules excited to a single dissociative continuum, the transition moment is either perpendicular or parallel to the recoil direction, yielding $\beta$ values of -1 and 2 , respectively. Intermediate values of $\beta$ result if the parent molecule has time to rotate before dissociating or if the bond which breaks is not strictly parallel or perpendicular to $\boldsymbol{\mu}$, which is generally true for polyatomic dissociations. The dependence of $\beta$ on excited state lifetime was first described in detail by Jonah [8] for diatomic molecules and was derived later by Yang and Bersohn [9] for polyatomics.

Translational spectroscopy is the most common method for measuring the photofragment angular distribution. This technique involves waiting for the fragments 
(a)

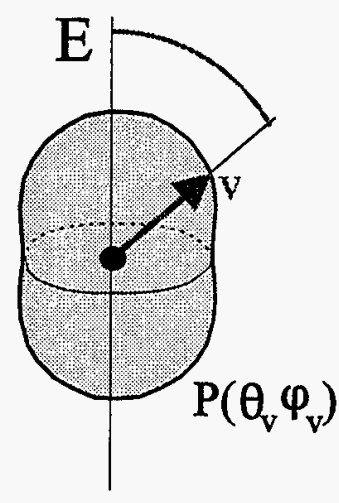

(b)

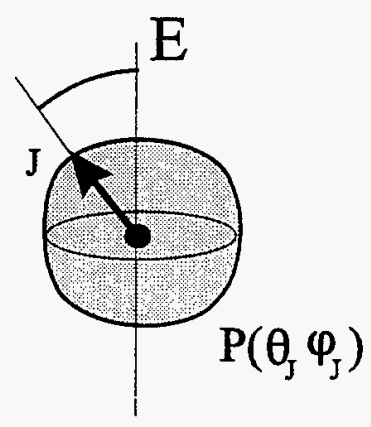

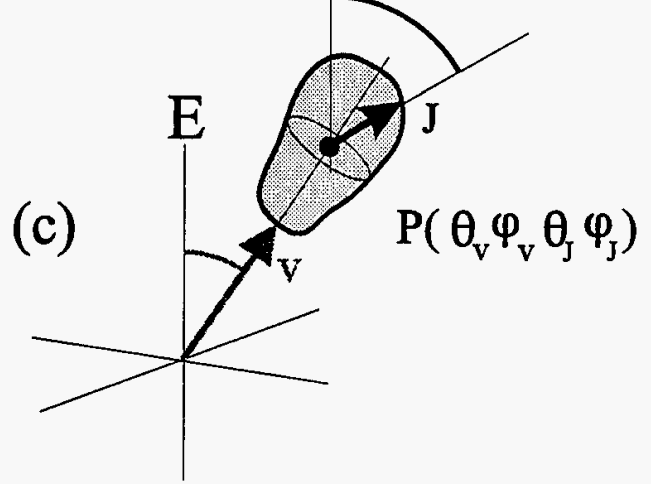

Figure 1.2: Distributions which describe vector correlations. (a) E-v correlation: 'velocity anisotropy' or 'photofragment angular distribution'. (b) E-J correlation: laboratory frame angular momentum polarization. (c) $\mathbf{v}-\mathbf{J}$ correlation. Note that according to these definitions, (a) and (b) are specific cases of (c).

to become separated in space due to differences in recoil speed and direction, and then detecting them as a function of position, time, and/or angle. The modern era of translational spectroscopy for the study of photodissociation started in 1969 with studies by Busch et al. [10] and by Diesen, Wahr \& Adler [11]. They used electron-impact ionization on a portion of the neutral photofragment cloud that had been cored by a small aperture to provide angular selectivity. In combination with a quadrupole mass spectrometer, this technique has the advantage of being able to individually detect all reaction products. For this reason, it remains popular today as a very general method for the study of photodissociation and bimolecular collisions [12]. More exotic neutral photofragment translational spectroscopies have led to breakthroughs in niche areas. Welge and coworkers developed a technique which uses field ionization of Rydberg-tagged hydrogen atom photoproducts to obtain unsurpassed kinetic energy resolution [13]. Studies of radical photodissociation [14] 
have been aided by neutralized fast ion beams in combination with sophisticated imaging detectors [15].

Other forms of translational spectroscopy, which use laser-based photofragment probes, have the advantage that they provide selectivity for individual photofragment quantum states, including angular momentum polarization. As of the late 1990s, the most commonly-used optical probe for translational spectroscopy is Resonanceenhanced Multiphoton Ionization (REMPI). The ion imaging technique described in this dissertation is an example of a REMPI-based translational spectroscopy. Other investigators have successfully used a variety of other coring, slicing and projection techniques $[16-20]$. It is also possible to probe the velocity vector distribution directly by using a narrow-bandwidth laser to measure Doppler profiles of individual fragment spectral lines, although strictly speaking this is not a translational spectroscopy. The use of Doppler lineshapes for the study of photodissociation was first discussed by Zare and Herschbach in 1963 [6]. Hall and Gordon have recently reviewed this widely popular method [21]. Fluorescence and ionization spectroscopies are the most widely-used probes for Doppler measurements, although a recent frequencymodulated absorption method has permitted measurements of photofragment vector correlations at an unprecedented level of accuracy [22].

\subsubsection{Angular Momentum Polarization}

Angular momentum polarization is another often-measured vector property. Like the velocity anisotropy, it is governed by excited state symmetries and lifetimes but provides a clearer window into the dynamics of transition-state nuclear motion. Early measurements focussed on angular momentum polarization in the laboratory frame (E-J correlation). Since the 1980s, the techniques to measure and interpret the more-detailed $\mathbf{v}-\mathbf{J}$ correlation have also become well-known, and today this is the observable of choice when studying angular momentum polarization in gas phase processes. The following overview of concepts and methods is applicable to both. The field of angular momentum polarization and vector correlations in general has been extensively reviewed in recent years [23-25]. 
(a)

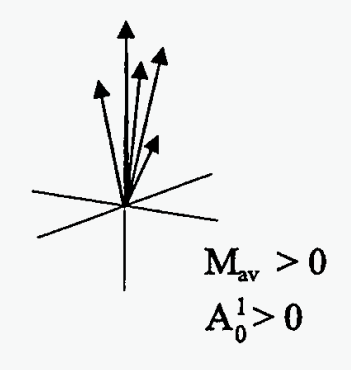

(b)

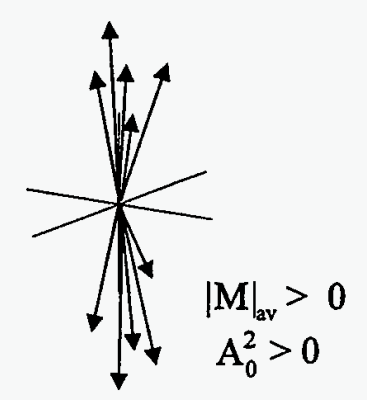

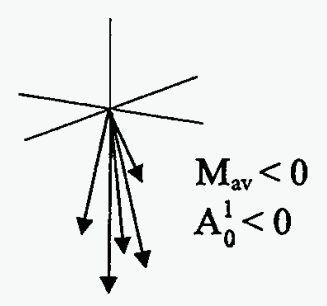

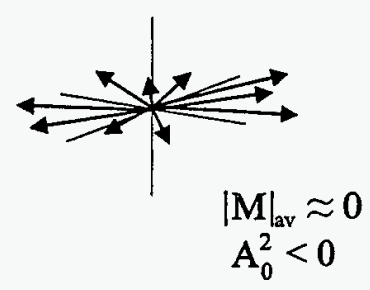

Figure 1.3: Examples of (a) oriented and (b) aligned ensembles of angular momentum vectors.

The angular momentum distribution gives the probability of the vector $\mathbf{J}$ pointing in a given direction. In the common terminology, alignment describes that portion of the distribution with inversion symmetry (e.g. $\mathbf{J}$ points more up and down than left and right), while orientation corresponds to the part which changes sign under inversion (e.g. J prefers to point up vs. down). Quantum mechanically, this corresponds to $\left|M_{J}\right|$-state selection vs. $M_{J}$-state selection, respectively. These distinctions are emphasized in Fig. 1.3.

The theoretical machinery for analyzing angular-momentum-polarized ensembles was first applied to experiments in atomic and nuclear physics during the 1950s [26]. It was not until the 1970s that these techniques found their way into the chemical physics community, largely by way of a review article by Fano and Macek [27]. Later articles by Case, McClelland and Herschbach [28] and by Greene and Zare $[29,30]$ further paved the way to widespread use. The common formalism describes the angular momentum distribution with a multipole expansion of the density matrix. In the classical limit of high angular momentum, this becomes an expansion 
of spherical harmonics, and the well-known orientation and alignment parameters $A_{q}^{k}$ are proportional to the expansion coefficients, i.e.

$$
A_{q}^{k} \propto\left\langle Y_{q}^{k}\left(\theta_{j}, \phi_{j}\right)\right\rangle^{\text {class }} \longleftarrow\left\langle\hat{J}_{q}^{k}\right\rangle^{Q . M .}
$$

where $k$ is odd for orientation and even for alignment, and the classical ensemble average is over the $\mathbf{J}$ distribution, with $\left(\theta_{j}, \phi_{j}\right)$ specifying the direction of $\mathbf{J}$. For the common case when the distribution is cylindrically symmetric, $q$ is equal to zero. Furthermore, one-photon dissociation limits $k$ to 0,1 or 2 , corresponding to photofragment population, dipolar orientation and quadrupolar alignment. Finally, as indicated in Eq. (1.2), the ensemble average is the classical limit of a density matrix average of the angular momentum spherical tensor operators $\hat{J}_{q}^{k}$.

Measurements of orientation and alignment with optical probes rely on the fact that anisotropy in the angular momentum distribution implies anisotropy in the ensemble of photofragment probe transition moments. A wide range of physical and chemical processes have been probed with optical techniques, including photodissociation, bimolecular collision and reaction, and surface scattering or desorption. Most early measurements were carried out by monitoring polarized fluorescence from electronically excited reaction products or Laser-Induced Fluorescence (LIF), and for the past decade, Resonance-enhanced Multiphoton Ionization (REMPI) schemes have also been widely used. To date, the large majority of experimental measurements of angular momentum polarization have focussed on the rotational angular momentum of diatomic reaction products. There has been particular emphasis on reaction products such as $\mathrm{NO}, \mathrm{OH}, \mathrm{CO}, \mathrm{CN}$ and $\mathrm{H}_{2}$, due both to their fundamental chemical importance and to the availability of convenient spectroscopic probe schemes.

\subsection{4 $\vec{v}-\vec{\jmath}$ Correlation}

The presence of net photofragment polarization in the laboratory frame requires the existence of the more detailed polarization of $\mathbf{J}$ about a specific recoil vector v. (Indeed, the former is just the recoil-angle average of the latter.) This fact was at least implicit in most discussions of photofragment angular momentum polarization 
prior to the first explicit measurements of the $\mathbf{v - J}$ correlation in 1986 [31-34], and there was often direct consideration of the processes which lead to molecular-frame angular momentum polarization. For example, Van Brunt and Zare discussed the polarization of atomic photofragments in terms of conservation of the orbital angular momentum projection along the bond axis [35]. For the dissociation of triatomic molecules, it was assumed (due to angular momentum conservation) that the diatomic fragment spins with its $\mathbf{J}$ preferentially aligned perpendicular to the recoil direction. This assumption permitted straightforward extraction of excited state lifetime and symmetry information from polarized fluorescence measurements (see e.g. Ref. [36]).

This dissertation adopts a very general but somewhat colloquial usage of the term ' $\mathrm{v}-\mathbf{J}$ correlation'. It is taken to be nothing more than the probability distribution $P\left(\omega_{v}, \omega_{j}\right)$, or in the language of scattering theory, a doubly differential cross section $\partial^{2} \sigma / \partial \omega_{v} \partial \omega_{j}$, where $\omega \equiv(\theta, \phi)$. The common narrower definition takes it to be that part of $P\left(\omega_{v}, \omega_{j}\right)$ which depends only on the angle $\omega_{v j}$ between $\mathbf{v}$ and $\mathbf{J}$. To avoid confusion, I will refer to this as the 'pure $\mathbf{v}-\mathbf{J}$ correlation'. The reason for this usage is to make a common term available to describe all vector correlations involving $\mathbf{v}$ and $\mathbf{J}$. Note that in the colloquial definition, the E-J and E-v correlations would be a subset of the $\mathbf{v - J}$ correlation-they correspond to those basis function components of $P\left(\omega_{v}, \omega_{j}\right)$ which are independent of $\omega_{v}$ or $\omega_{j}$, respectively.

The concept of the $\mathbf{v}-\mathbf{J}$ correlation allows a discussion of dynamics from the perspective of the recoil vector, which is more closely related to the molecular frame and thus conceptually and theoretically convenient. Given that the E-v and E-J correlations both occur via an intermediate correlation with the parent transition moment, it is not surprising that $\mathbf{v}$ and $\mathbf{J}$ should be correlated to each other. The pure $\mathbf{v}-\mathbf{J}$ correlation even survives rotational scrambling of the nascent polarization of the parent molecules, since it results only from torques which act at the moment of dissociation. In prompt dissociations, valuable information may be obtained if the shape of the angular momentum distribution in the recoil frame is found to vary with recoil angle. This is an indicator that multiple excited states and possibly coherence effects contribute to the dissociation process.

The general goal of a mathematical description of the $\mathbf{v}-\mathbf{J}$ correlation is to 
derive the recoil angle dependence of the angular momentum polarization, described by parameters such as a density matrix or its multipoles. Again, these techniques arrived in the chemical physics community via spinoff from nuclear physics of decades earlier [37]. The most popular approach for treating photodissociation comes from Dixon [38] and appeared shortly after the first explicit measurements in 1986. In this method, the distribution function $P\left(\omega_{v}, \omega_{j}\right)$ is expressed as an expansion of bipolar harmonics, which are a convenient basis set to describe correlations between two vectors. Dixon derived semiclassical multipoles as a function of Doppler shift and then applied standard techniques for calculating fluorescence signals. This method has the advantage that all vector correlations, including velocity anisotropy, are treated systematically in a unified approach. Moreover, each bipolar moment has a straightforward geometric interpretation which leads relatively easily to dynamical interpretations.

For low values of the angular momentum quantum number, a fully quantummechanical treatment is necessary. Dixon's treatment is semiclassical in the sense that the angular distribution of $\mathbf{J}$ is continuous, however it is possible to modify it for low-J systems. Others have derived fully quantal descriptions of the $\mathbf{v}-\mathbf{J}$ correlation. Hall et al. [39] presented an early density matrix treatment, however it lacked a systematic means for fitting data and contained the unrealistic approximation that the shape of the angular momentum distribution was independent of recoil angle. A very recent treatment of fluorescence imaging by Chen et al. [40] gives expressions in terms of density matrix multipoles. While this treatment has much in common with the method discussed in this thesis, it provides no description or interpretation of the recoil angle dependence of the angular momentum polarization. Of more relevance for our study is a recent quantal treatment by Siebbeles et al. [41], which gives expressions for angle-dependent density matrix multipoles in terms of scattering matrix elements. The results of this work were central to the analysis contained in this dissertation.

Experiments which combine velocity-resolving techniques with optical probes are sensitive to the $\mathbf{v}-\mathbf{J}$ correlation. LIF Doppler profile measurements were most common throughout the 1980s, although in recent years, REMPI-based techniques have become at least as popular. Briefly, the general principle which applies to all 
such measurements is as follows. The nascent photofragment ensemble has an angular shape (in space, following expansion due to recoil), which is determined by the velocity anisotropy alone. In contrast, the shape of the optically-probed photofragment ensemble will have additional angular modulations due to the $\mathbf{v}-\mathbf{J}$ correlation. This post-probe shape is measured with the chosen position- or velocity-sensitive technique. In order to fully characterize the distribution $P\left(\omega_{v}, \omega_{j}\right)$ and to isolate the small modulations from the much larger total signal, one contrasts data collected under different probe conditions. For example, one may select different probe polarizations (corresponding to different transition moment projections) or different optical transitions (corresponding to different transition moments).

\subsection{Atomic Angular Momentum Polarization}

The angular momentum polarization of atomic photofragments provides information about the electronic rearrangement which occurs during excitation and dissociation. An elementary molecular orbital description of chemical bonding suggests that anisotropy of the electron density in a photoexcited molecule should correlate to anisotropy in the resulting atom(s) (Fig. 1.4). For example, a broken pi-bond will lead to half-filled $p_{x}$ or $p_{y}$ orbitals and thus a net polarization of orbital angular momentum $\mathbf{L}$. Although it captures the essence of the effect, the one-electron molecular orbital picture is oversimplified, since individual electron orbital angular momenta $\mathbf{L}$ are coupled to each other and to spins to yield total atomic angular momentum $\mathbf{J}$. Nevertheless, although we ultimately measure the polarization of $\mathbf{J}$, it is quite common and reasonable to refer to the effect as 'orbital polarization', since the mechanism relies predominantly on the electric fields associated with the excitation/dissociation process. Spin is not directly influenced by these electric fields but may be involved through spin-orbit coupling. In the end, the term 'orbital polarization' should not be discarded for being slightly simplistic, since it is far more meaningful to non-specialists than 'total electronic angular momentum polarization'.

The first discussion of a mechanism for production of polarized atomic photofragments appeared in the 1968 paper of Van Brunt and Zare [35], and it was 
Molecular
States

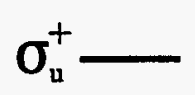

Molecular

Orbital

Diagram

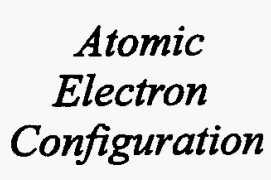

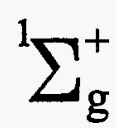

$\Pi^{1}{ }^{3} \Pi_{u}$

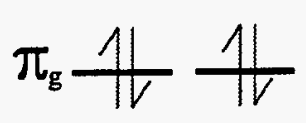

$\pi_{\mathrm{u}} \frac{11}{1 \mathrm{~V}} \frac{1}{\mathrm{IV}}$

$$
\sigma_{\mathrm{g}}^{+} \frac{11}{1 /}
$$

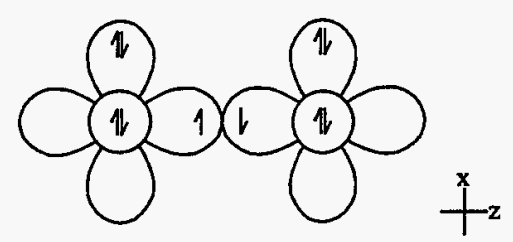

Half-filled

$\mathrm{p}_{\mathbf{z}}$ orbitals
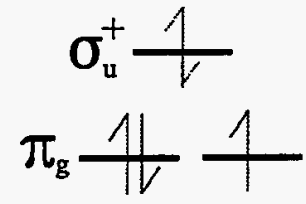

$\pi_{u} \frac{11}{1 V} \frac{1}{16}$

$$
\sigma_{\mathrm{g}}^{+} \frac{1}{1 V}
$$

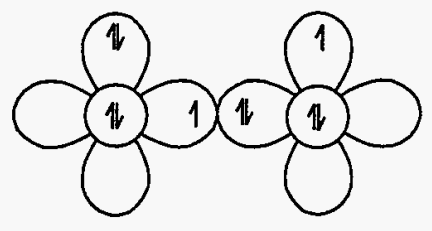

Half-filled $\mathrm{p}_{z}$ and $\mathrm{p}_{\mathrm{X}}$ orbitals

Figure 1.4: Molecular orbital view of atomic photofragment polarization-e.g. diatomic halogen. The ground molecular electronic state leaves 'holes' in atomic $p_{z}$ orbitals only, while states corresponding to the first excited electron configuration yield both $p_{z}$ and $p_{x}$ or $p_{y}$ holes. Half-filled atomic orbitals result in net angular momentum polarization.

not until 1980 that the first positive experimental observations were reported [42, 43]. (A pioneering early 'negative' result [44] was finally explained by theory only a few years ago [45]). Progress in this area since then has clarified the role of several factors which determine the atomic photofragment polarization. A summary of the contributing factors is shown in Fig. 1.5. In addition to the role of the initial state symmetry, which is emphasized by the molecular orbital viewpoint, one must also consider the possibility of coherent excitation to multiple electronic states, nonadiabatic transitions, and the long-range interactions between the departing photofragments.

When only a single adiabatic potential energy surface is involved in the dissociation, the atomic polarization is determined by the symmetry of the dissociative 


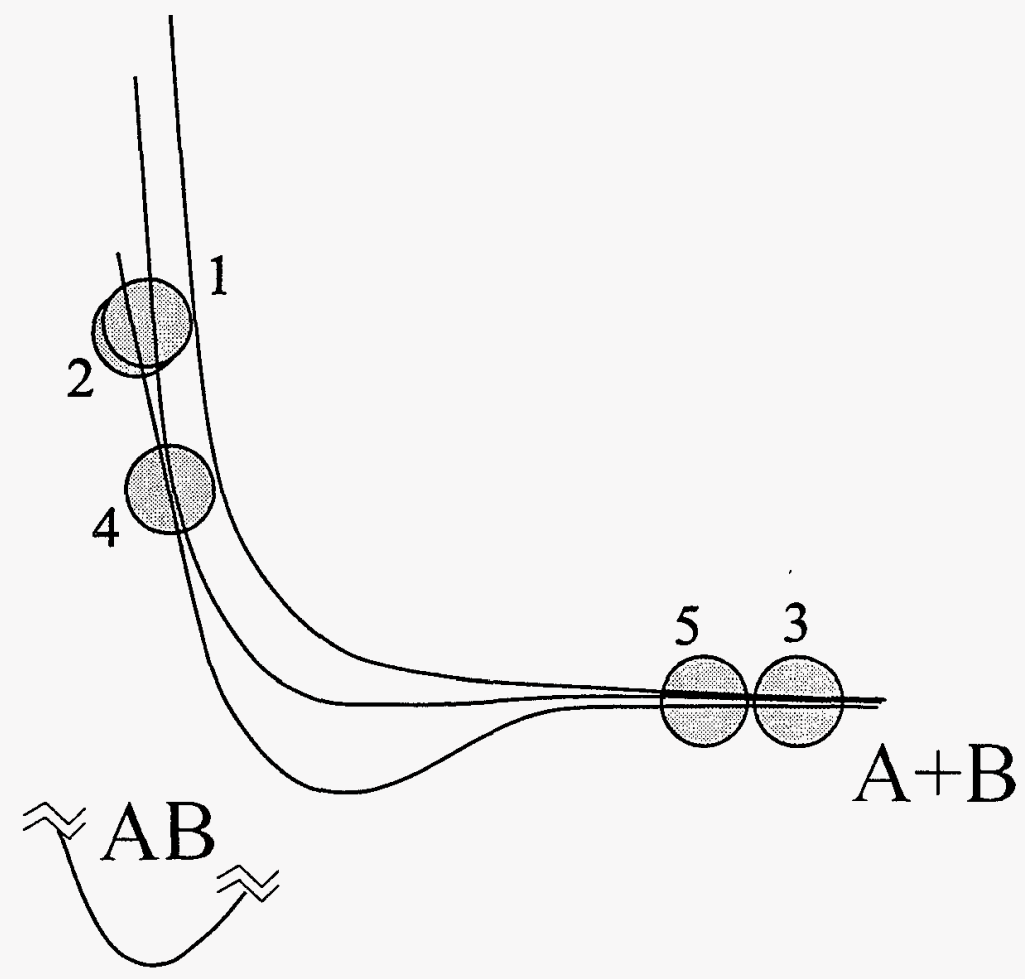

Figure 1.5: The factors which affect the polarization of atomic photofragments occur in different regions of the molecular potential energy curves: (1) Symmetry of excited electronic state. (2) Coherent excitation of multiple electronic states. (3) Coulomb interaction at large internuclear separation and corresponding adiabatic correlations to molecular region. (4) Nonadiabatic transitions at avoided crossings. (5) Nonadiabatic transitions at large internuclear separation.

electronic state and the corresponding long-distance interaction between the recoiling atoms. Van Brunt and Zare predicted an anisotropic distribution of fluorescence from the photoproducts of diatomic dissociations-a consequence of polarized atoms and an anisotropic photofragment angular distribution. The atomic polarization results from the approximate conservation of the projection of the total angular momentum on the bond axis during the course of dissociation:

$$
\Omega_{A B}=\left|M_{A}+M_{B}\right| .
$$

Thus, according to Van Brunt and Zare, "molecular dissociation can be viewed as a highly efficient atomic $M$-state selector." A more detailed description of adiabatic dissociation must consider the long-range Coulomb interactions between the depart- 
ing atoms [45]. Vasyutinskii and Beswick are currently preparing a review of this topic [46]. Perturbation theory shows that different atomic polarizations will result from adiabatic states of the same symmetry which are degenerate at large internuclear distance. As a consequence, one must consider not only the state symmetry, but its ordering among other states of the same symmetry.

In general, multiple adiabatic potential energy surfaces will be involvedin the initial excitation and through nonadiabatic transitions during the course of dissociation. In the initial photon absorption, coherent excitation of multiple electronic states will affect the polarization of atomic photofragments. The quantummechanical interference which results from coherent excitation is observable in both the total polarization and in the polarization angular distribution, though the latter will provides more detailed information about the excited states. The importance of coherence effects became clear in 1981 when Vigué and coworkers [47] reported polarized photofragment fluorescence for $\mathrm{Ca}_{2}$ dissociation, which was far in excess of the maximum allowed by the Van Brunt and Zare theory. This result could only be explained as a coherent excitation to the degenerate $\Omega_{e x}= \pm 1$ 'lambda-doublet' components of a ${ }^{1} \Pi_{u}$ state. A second type of coherence effect involves excitation to two states of different symmetry $\left(\Omega_{e x}=1\right.$ and 0 ) via simultaneous parallel and perpendicular excitation. This process, first discussed by Vasyutinskii in 1986 [48], requires two nearby electronic states with comparable absorption cross sections. Beswick and Glass-Maujean have provided detailed theoretical descriptions of both types of coherence effects $[49,50]$.

Nonadiabatic transitions between electronic states of the same symmetry can either increase or decrease the degree of polarization relative to that of an adiabatic dissociation. In general, we are concerned with nonadiabatic transitions in two regions. The most familiar type occurs at localized 'avoided crossings' within the range of internuclear distances where exchange interactions are important and the Born-Oppenheimer approximation is otherwise valid. The second type of transition occurs at large internuclear distance where the adiabatic states are nearly degenerate, and the Born-Oppenheimer approximation is not valid. An extensive theoretical treatment of this problem was presented by Singer et al. in 1983 [51]. This study de- 
rived expressions for polarized fluorescence and for the angular distribution of atomic fine-structure (spin-orbit) states in the dissociation of diatomic molecules.

Already in 1968, Van Brunt and Zare hinted at the value of $\mathbf{v - J}$ correlation measurements:

Combined polarization and Doppler width measurements can provide, of course, additional information in favorable cases, but with a corresponding increase in experimental difficulties. [35]

The value of such measurements was explicitly laid out in the 1994 theoretical paper by Siebbeles et al. [41]. As mentioned previously, this work provides expressions for angle-dependent density matrix multipoles, $\rho_{k q}(\theta, \phi)$, in terms of scattering amplitudes. Parallel and perpendicular optical excitations and coherence effects are each shown to have a unique influence on the angular distributions of orientation and alignment. This paper provides, in principle, the means for experimentalists to extract meaningful dynamical information from $\mathbf{v}-\mathbf{J}$ correlation measurements.

By the mid-1980s the 'experimental difficulties' of $\mathbf{v}-\mathbf{J}$ correlation measurements mentioned by Van Brunt and Zare had largely been overcome, although it was nearly a decade more before these techniques were applied to atomic polarization. In the field of chemical reaction dynamics, the bulk of experimental studies of angular momentum polarization have dealt with the polarization of rotating diatomic photofragments. This is not surprising, since this field is concerned largely with the dynamics of nuclear motion governed by a potential energy surface. Although a small group of specialists have studied atomic photofragment polarization since the early 1980 s, it is only quite recently that detailed interest in this field has diffused to the broader group. Within the past three years there have been at least six reports (formal and informal) of atomic photofragment polarization measured as a $\mathbf{v}-\mathbf{J}$ correlation. The earliest report outside of the original community of specialists was in 1993 for polarized $\mathrm{O}\left({ }^{1} \mathrm{D}_{2}\right)$ produced by ozone dissociation at $205 \mathrm{~nm}$ [52]. Since then, there have been reports of polarized $\mathrm{O}\left({ }^{1} \mathrm{D}_{2}\right)$ from $\mathrm{N}_{2} \mathrm{O}$ [53], and $\mathrm{S}\left({ }^{1} \mathrm{D}_{2}\right)$ from OCS [54]. The polarized chlorine atom result, first reported by Wang et al. [55], was also observed in 1995 by two other groups $[56,57]$. Given this wider exposure in the last few years, it is likely that there are more studies underway. 
It is interesting to consider why this topic is only now receiving wider attention outside of the original group of specialists -15 years after the first observations of polarized atomic photofragments, and nearly 30 years after the prediction of Van Brunt and Zare. Perhaps the most important factor is that until the late 1980s, there were very few measurements on atomic photofragments which used angularmomentum-sensitive optical techniques. During the first ten years of widespread use of laser-based methods, most investigators were primarily interested in obtaining quantum state populations. Diatomic fragments, with their many accessible quantum states, were the most interesting from this perspective. Those who studied angular momentum polarization used it largely as an alternative means for measuring the velocity anisotropy -an emphasis which even dominates Van Brunt and Zare's discussion of atomic polarization. Before the velocity-sensitive Doppler and REMPI-based time-of-flight techniques became widely available, there simply was not much of a reason to detect atoms with laser techniques. The ability to directly measure the velocity anisotropy with these techniques provided a new motivation to probe atomic photoproducts. The appearance of REMPI-based ion imaging in the late 1980s was a particularly important innovation, since it allowed velocity-sensitive measurements without the specialized narrow-band laser systems required for Doppler measurements.

Now in the mid-1990s, with many investigators using laser-based techniques to study atoms, it has become quite clear to most that these methods are sensitive to $\mathbf{J}$ as well as to $\mathbf{v}$. Even if investigators are not specifically interested in the atomic polarization, they must know how to treat it in their data analysis to properly extract velocity anisotropies and speed distributions. A similar realization occurred for $\mathbf{v - J}$ correlations of diatomic photofragments a decade ago. Hall and coworkers commented at that time,

"The presence of the $\mathbf{v}-\mathbf{J}$ angular correlation can be both a benefit and an annoyance. Measurement of the angular correlation provides a new dynamical variable which can help in the understanding of chemical processes. On the other hand, neglect of its effect on the Doppler profile can easily produce misleading results." [31] 
With these considerations in mind, it is heartening to look back with hindsight at the evolution of the diatomic $\mathbf{v}-\mathbf{J}$ correlation. While it might formerly have been considered an 'annoyance', the diatomic $\mathbf{v}-\mathbf{J}$ correlation has since become a powerful observable for disentangling the details of photodissociation dynamics. This change depended on having the 'annoyance' standing in the path of a wider group of investigators, combined with a core of specialists intent on figuring it out. Similar events seem to be occurring for the atomic $\mathbf{v}-\mathbf{J}$ correlation, and if the past is any indication, there should be more exciting developments in the next several years. 


\section{Chapter 2}

\section{Experimental Methods and Principles}

\subsection{Overview}

The experiments discussed in this dissertation involve measurement of the correlated angular momentum and velocity vector distribution for atomic photofragments. We probe the former with a quantum-state-selective spectroscopic technique called Resonance-enhanced Multiphoton Ionization (REMPI). The velocity vector distribution is measured with the photofragment ion imaging technique. This is a form of translational spectroscopy which maps the three-dimensional velocity distribution of REMPI ions into a two-dimensional spatial distribution. The signature of the $\mathbf{v - J}$ correlation is a spatial modulation in the ion image which depends on the REMPI transition and probe laser polarization. The initial section of this chapter considers the experimental approach as a whole, including some details not discussed elsewhere. The REMPI and ion imaging methods are each covered in detail in separate sections. The final section is a qualitative description of their combined use for $\mathbf{v}-\mathbf{J}$ correlation measurements.

The ion imaging technique was first reported in the literature by Chandler and Houston [58]. In applying the new technique to the much-studied dissociation of methyl iodide, they demonstrated the power of measuring the recoil velocity dis- 
tribution for a specific photofragment quantum state. Although Doppler profiles had previously been shown to provide similar information, ion image data are a two-dimensional rather than one-dimensional projection of the spatial distribution of photofragment ions, making it more straightforward to reconstruct the original three-dimensional ion distribution, and thus the energy and angular distributions of the neutral photofragments. For cylindrically symmetric distributions, such data may be inverted directly with the inverse-Abel transform [59]. Finally, one should not understate the benefit of an image's visual appearance, which is more intuitively meaningful than a one-dimensional projection. In the past decade, ion imaging has been widely-used for photodissociation and shows promise for more extensive use in the study of bimolecular reactions. Three recent articles give an extensive overview of the ion imaging technique and the chemical systems which have been studied with it [60-62].

Fig. 2.1 shows a schematic diagram of the key components of our experimental apparatus. A summary of the technique is provided here, and the various components are described in turn in the remainder of this chapter. A standard ion imaging apparatus consists essentially of a time-of-flight mass spectrometer (TOFMS) and a position-sensitive ion detector. In each cycle of the experiment (running at $10 \mathrm{~Hz}$ ), a pulsed supersonic expansion of the gas molecules of interest is crossed at $90^{\circ}$ by counter-propagating laser beams. The first laser pulse dissociates a small fraction of the molecules contained in the intersection volume of the laser and the molecular beam. After a short delay (10-50 ns), a 2+1 REMPI process state-selectively ionizes the nascent photofragments. The ions resulting from each laser shot are extracted by an electric field into a grounded flight tube. In order to keep the interior of the flight tube field free, both ends of the tube are isolated from the high voltage regions with fine nickel mesh (100 lines per inch). During its flight time, the chlorine ion packet expands due to the recoil energy remaining after dissociation. At the end of the flight tube, the expanded packet strikes the surface of a microchannel plate, which is gated to detect only the mass of interest. Electrons emerging from the back of the microchannel plate impinge on a phosphor screen to produce images which are collected with a video camera and signal-averaged. 


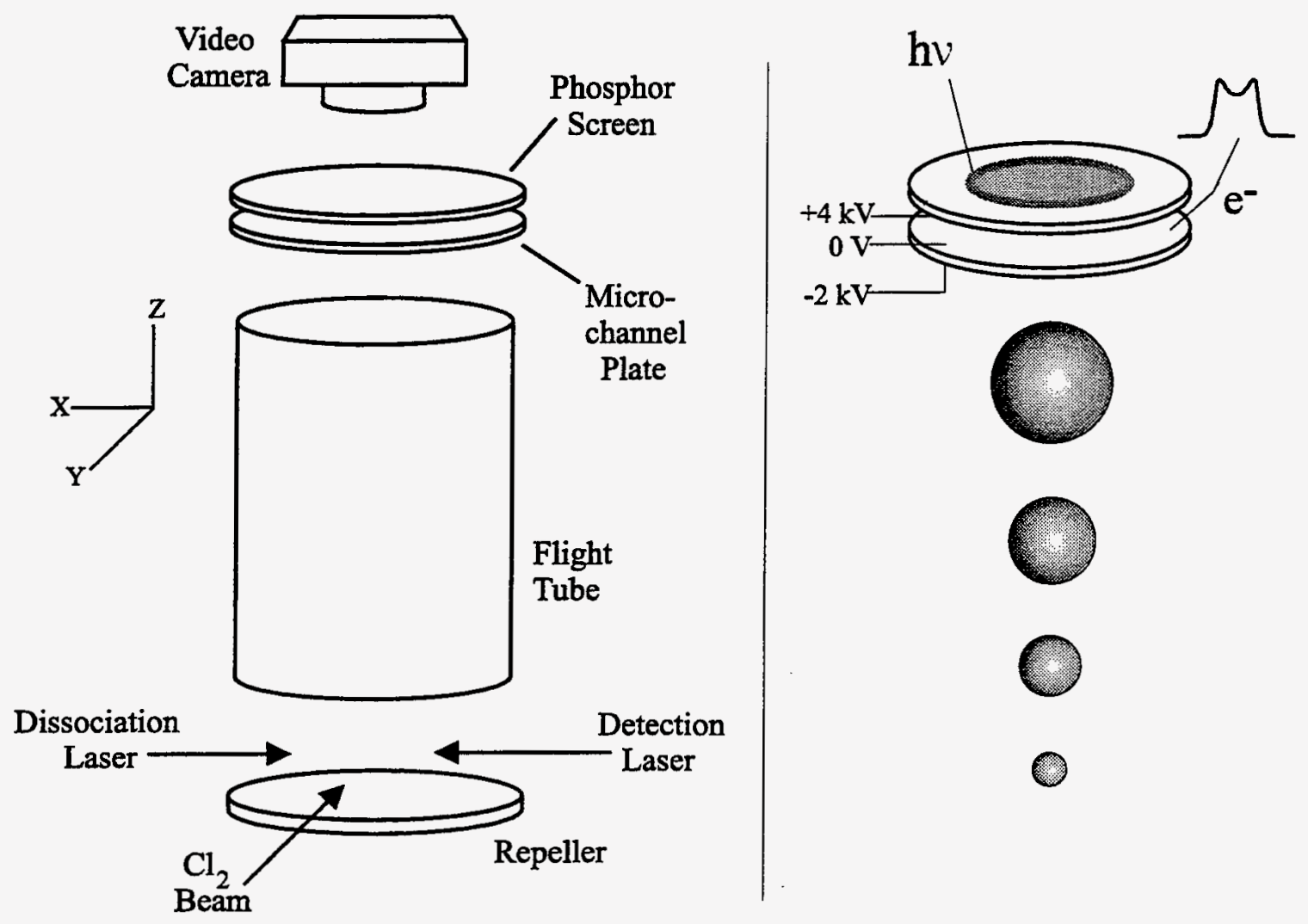

Figure 2.1: Schematic of an ion imaging experiment. At right, the basic principle of the method is shown. See Sec. 2.3.2 for further information. By directly collecting electrons, TOFMS peak profiles may also be measured, although this is typically done with a different detector. In general, the ion packet is an ellipsoid (rather than a sphere) by the time it reaches the detector. 
The experimental apparatus as a whole is shown in Fig. 2.2. The interaction region is located at the center of a vacuum chamber which is pumped with a $2000 \mathrm{l} / \mathrm{s}$ turbomolecular pump. The near absence of diffusion pump oil vapor in the main chamber has three advantages for a REMPI-imaging experiment-it reduces the nonresonant background ion signal, prevents oil coatings on the extraction optics which lead to surface charging, and it extends the lifetime of the microchannel plate detector. The detector region itself is a separately-pumped chamber which may be isolated with a gate valve from the main chamber in order to maintain vacuum during routine vents, prolonging the lifetime of the microchannel plate. For use in core-sampling experiments (see Chapter 5), an xyz manipulator is mounted on the detector region in order to position an aperture in the middle of the ion cloud flight path.

The pulsed molecular beam is created in a third chamber, which is pumped by a large diffusion pump. Gas samples are introduced to a pulsed molecular beam valve [63], which uses a piezoelectric actuator to retract an oring from a small nozzle opening, allowing gas to expand into the vacuum for roughly $200 \mu \mathrm{s}$. The resulting free jet expansion is collimated by a conical skimmer which also serves to deflect the excess gas away from the rest of the pulse. Typically, experiments are carried out on the 'rising edge' of the pulse, at a delay of roughly $50 \mu \mathrm{s}$ after the weakest signals are first detected, although earlier delay times are sometimes used if cluster formation is evident. The beam 'temperature' was not explicitly characterized for the experiments described in this thesis, although translational and rotational temperatures of several Kelvin are typical for helium-seeded expansions.

\subsection{State-selective Spectroscopic Detection with REMPI}

\subsubsection{General Considerations and Applications of REMPI}

With the widespread availability of high-power tunable pulsed lasers in the late 1970s, powerful new detection techniques came into the mainstream. Chief among 


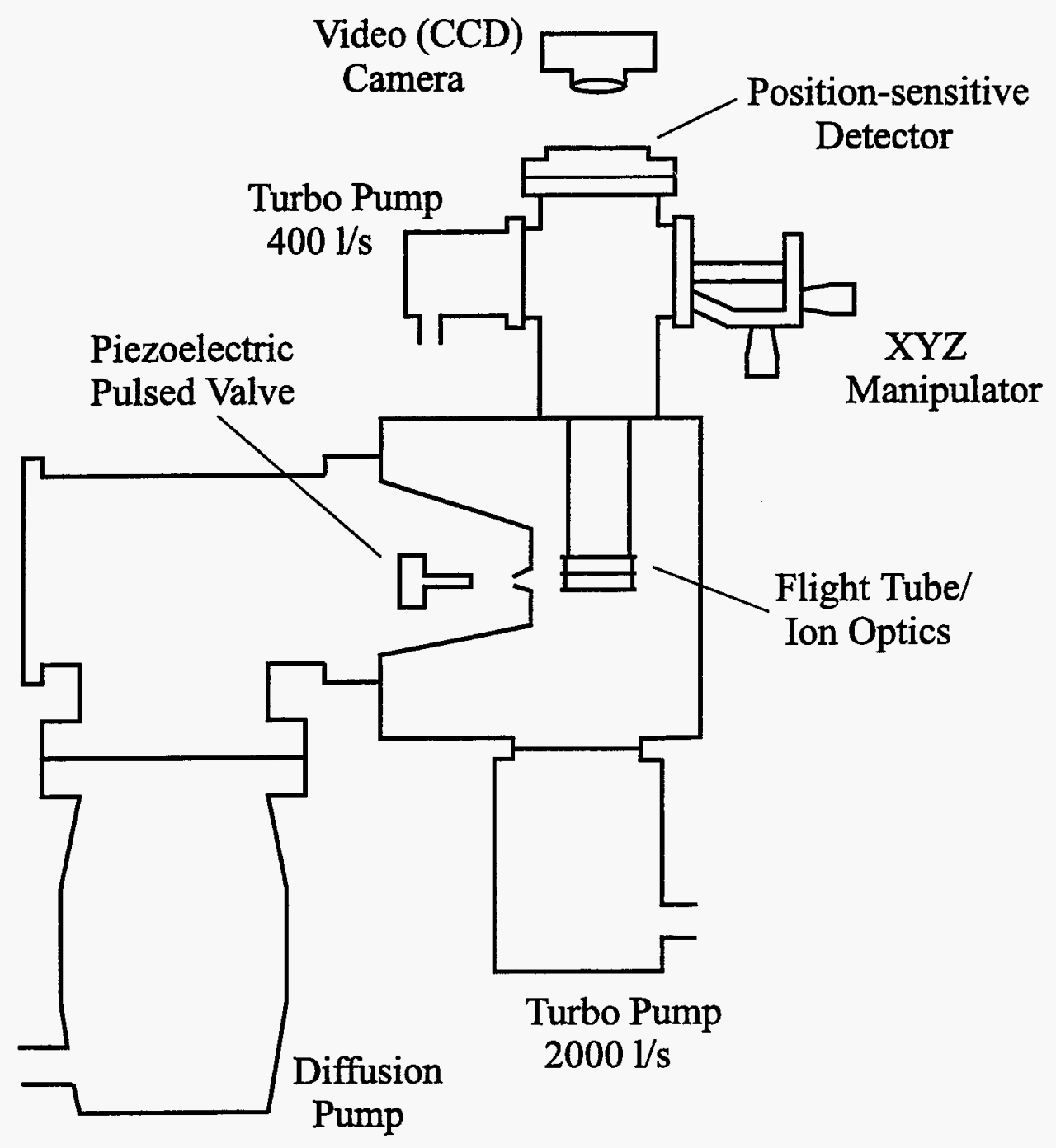

Figure 2.2: Schematic of experimental apparatus. See text for details. 
these is the use of multiphoton ionization as a quantum state-specific probe. In $\mathrm{N}+\mathrm{M}$ Resonance-enhanced Multiphoton Ionization (N+M REMPI), there are two resonant steps $-\mathrm{N}$ photons excite an atom or molecule to an electronically-excited eigenstate, and $\mathrm{M}$ photons ionize it. In 1+1 REMPI, moderately high power densities are necessary to drive the ionization step, which is often in competition with decay processes such as fluorescence and predissociation. As a probe for studying chemical dynamics, $1+1$ REMPI is used less than higher order $(\mathrm{N}>1)$ schemes, since it often involves the additional challenge of producing vacuum ultraviolet photons. (Nitric oxide is one major exception).

Higher power densities are needed if either resonant step involves more than one photon. Multiphoton absorption is predicted by second-order (and higher) timedependent perturbation theory, which shows that the transition probability increases with the Nth power of the laser field intensity. Each non-resonant single photon 'absorption' relies on oscillator strength from one-photon-allowed transitions which may be thousands of $\mathrm{cm}^{-1}$ displaced from the photon energy. (As discussed in the next chapter, these 'virtual' states influence the sensitivity of the REMPI scheme to angular momentum polarization.) 2+1 REMPI schemes for a variety of common species are available in the near ultraviolet (above $200 \mathrm{~nm}$ ). These wavelengths are readily produced from visible dye laser light by using common nonlinear optical mixing techniques.

The fact that optical spectroscopies are sensitive to the direction of the angular momentum vector is clear from a classical perspective: a specific torque is needed to change the length or direction of the angular momentum vector in a chosen manner. In more precise language, the transition dipole lies in a well-defined direction with respect to the angular momentum vector: a change $\delta \mathbf{J}$ in angular momentum is associated with a dipole rotating in a plane perpendicular to $\delta \mathbf{J}$ (Fig. 2.3a). For example, the transitions shown in Figs 2.3(b) and (c) have transition dipoles roughly parallel and perpendicular to $\mathbf{J}$, respectively. The first transition is more sensitive to high- $\left|M_{J}\right|$ states, while the second is more sensitive to low- $\left|M_{J}\right|$ states. $\left(M_{J}\right.$ is defined here as the projection of $\mathbf{J}$ on $\mathbf{E}_{\text {probe. }}$ )

Given the above considerations, there are two measurable signatures of an- 


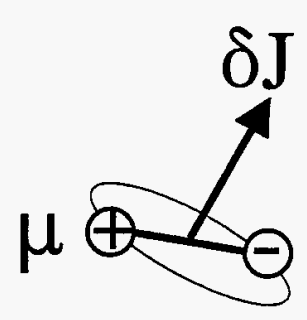

a.

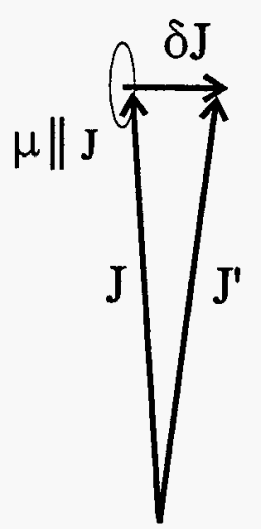

b.

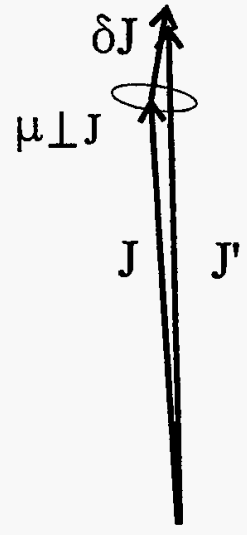

c.

Figure 2.3: Relationship of $\boldsymbol{\mu}$ to $\mathbf{J}$. (a) Change in $\mathbf{J}$ is perpendicular to dipole plane of rotation. (b) Example: Transition dipole parallel to J. (c) Example: Transition dipole perpendicular to $\mathbf{J}$.

gular momentum polarization. For a fixed choice of spectroscopic transition, one may look for a change in signal intensity as the probe light polarization is rotated, in effect measuring different projections of the transition moment ensemble. For total polarization measurements (independent of recoil direction), this is the most common method. One also has the option of looking for a change in signal when a different spectroscopic transition is chosen (with a correspondingly different anisotropic distribution of transition moments). However, this approach is inconvenient for measurements of absolute signal size (e.g. to determine total angular momentum polarization), since transition linestrength factors will be different and some experimental conditions are likely to change when the wavelength is tuned. It is quite convenient for $\mathbf{v}-\mathbf{J}$ correlation measurements however, since one looks for a change in the shape of a signal distribution such as a Doppler profile, TOFMS profile or ion image. These ideas are developed in more detail in Sec. 2.4 and in Chapters 3 and 4.

Two-photon-resonant excitation permits one to obtain a more complete description of a polarized ensemble. In general, more photons in the probe process permit sharper resolution of the angular momentum distribution. The optical dipole nature of the probe limits one to measuring moments of rank $2 \mathrm{~N}$ or less, where $\mathrm{N}$ is the number of photons in the probe process. Linearly polarized light is capable of de- 
tecting only even rank moments (alignment), while circularly or elliptically polarized light may detect both even and odd (orientation) moments. Note that for most $\mathrm{N}+1$ REMPI schemes with $\mathrm{N}>1$, the one-photon ionization step is easily saturated and thus insensitive to angular momentum polarization. For this reason, 2+1 REMPI can be treated as a two-photon scheme. It is capable of detecting moments up to rank 4, i.e. contributions as 'sharp' as $g$-orbital shapes.

However, there are fundamental limits to how sharply a distribution may be peaked, limiting the usefulness of additional probe photons. Quantum mechanical uncertainty imposes an upper limit of $2 J$ on the rank $k$ of the distribution. This is seldom important for measurements on diatomic photofragments, where $J$ is usually large, but it matters for atoms. For example, the $J=3 / 2$ chlorine atom photofragments discussed in this thesis may show quadrupole alignment $(k=2)$, but not hexadecapole $(k=4)$ alignment. Other restrictions exist as well. When the entire photofragment ensemble is considered, the same $2 \mathrm{~N}$ limit discussed above for the probe process also applies for the dissociation process, i.e. single-photon dissociation can only produce up to rank 2 alignment. In addition, cylindrical symmetry (projection $q=0$ ) is required if the light polarization is also cylindrically symmetric (linear or circular). Thus the $\mathbf{J}$ distribution for a single photon dissociation with linear polarization can be completely described by the simple form $P\left(\theta_{J}\right) \propto 1+\frac{5}{2} A_{0}^{(2)} P_{2}\left(\cos \theta_{J}\right)$, where $A_{0}^{(2)}$ is the usual alignment parameter.

The situation is much less restrictive for the $\mathbf{v}-\mathbf{J}$ correlation-we can expect more sharply peaked angular momentum distributions at a single recoil angle than for the photoproduct ensemble as a whole. This agrees with our intuition, for example, of a dissociating triatomic molecule-the torque on the diatomic photofragment is in the same plane as the molecule and the velocity vector $\mathbf{v}$, so $\mathbf{J}$ for the diatomic fragment must be strictly perpendicular to $\mathbf{v}$. As shown in the theoretical analysis of Siebbeles et al. [41], all combinations of the indices $k$ and $q$ (i.e. $k=0 \ldots 2 j, q=-k \ldots k$ ) can be nonzero. Even for linearly polarized dissociation light, it is possible to produce angle-dependent multipoles with odd $k$ (orientation). Given these considerations, the additional angular resolution provided by multiphoton probes can be beneficial. It should be added, however, that in many cases the lowest order moments contain 
the bulk of the desired dynamical information, so the additional advantage is often small. Given the complications of analyzing two-photon data, it should be plainly stated that the most important advantage of multiphoton excitation schemes is their abundance and ease of implementation.

\subsubsection{Producing and Using Ultraviolet Laser Light}

By the middle of the 1990s, generation of tunable high power coherent ultraviolet light has become routine. Frequency mixing of visible pulsed dye laser light remains the workhorse technique for many, although solid state tunable lasers in the red and infrared are becoming more widespread. This section provides brief details on the generation and application of ultraviolet light in a 2+1 REMPI experiment. A more detailed discussion of UV generation for REMPI experiments can be found in $\operatorname{Ref}[64]$.

The laser performance requirements for a typical photodissociation imaging experiment are not especially demanding. For ionization-based spectroscopy, velocity information can be obtained from the spatial separation of the ions, and thus narrowbandwidth lasers for Doppler scans can be avoided. In fact, in an imaging experiment, when the laser bandwidth is comparable to or less than the Doppler width, the laser frequency must be scanned during data collection to sample all velocities. The limited bandwidth demands of a non-Doppler experiment represent a significant benefit in terms of lower cost and technical simplicity of operating and maintaining the laser system. It should be mentioned, however, that some investigators have used novel combinations of sub-Doppler REMPI with core-sampling TOF techniques to directly measure doubly-differential cross sections [17].

The experiments described in this thesis were carried out using pulsed Nd:YAG-pumped dye lasers. Although some of the measurements were made with a modern laser (Laser Analytical Systems), a number of measurements were also done with an older and much simpler Spectra Physics PDL-1. In some ways, the PDL-1 is preferable for imaging experiments because of its broad bandwidth and simple design. The LAS laser provides narrow bandwidth $\left(\sim 0.04 \mathrm{~cm}^{-1}\right.$ in the visible), a cleaner 
spatial mode, and a more sophisticated user interface, but only the latter is relevant for an imaging experiment, and ultimately the PDL-1 is a more effective 'workhorse' system requiring less attention in the long run. (Of course, very few laboratories are designed exclusively around the ion imaging technique, so the above-mentioned criteria are far too limiting in general.)

Fig. 2.4 summarizes the REMPI schemes used in this work. In all experiments, dye lasers were pumped with an unseeded Nd:YAG laser, which produced pulses of 8-10 ns at $532 \mathrm{~nm}$. The dye laser output was frequency-doubled (or sometimes mixed with the Nd:YAG fundamental) with a KDP crystal, and the resulting doubled (or mixed) light was mixed again in a BBO crystal to produce the ultraviolet REMPI probe light. Pulse energies were typically 30-100 mJ for the initial visible dye laser light and between $50 \mu \mathrm{J}$ and $2 \mathrm{~mJ}$ for the final ultraviolet light. Conversion efficiencies for the various nonlinear mixing process were low, due primarily to the poor spatial mode of the visible laser light, and in part to optical damage in the non-linear crystals. Nevertheless, the final UV powers were sufficient for $2+1$ REMPI.

Focussing conditions for both the photolysis and REMPI lasers are usually chosen so as to avoid saturation of either the parent absorption or the 2-photon atomic fragment absorptions, while still saturating the ionization step of the 2+1 REMPI process. The dissociation step should not be saturated, since 'hole-burning' due to the $\boldsymbol{\mu} \cdot \mathbf{e}$ absorption probability would create an undesired spatial anisotropy in the parent ensemble. In our experiments, typical photolysis pulse energies were $0.2-1.0 \mathrm{~mJ}$ and the beam was focussed away from the interaction region to give a spot size of at least $200 \mu \mathrm{m}$. For chlorine dissociation at $355 \mathrm{~nm}$, which has an absorption cross section of $2 \times 10^{-19} \mathrm{~cm}^{2}$, these conditions result in absorption by less than $10 \%$ of the ground state molecules.

In the REMPI process, only the unsaturated two-photon step should be sensitive to angular momentum polarization. Given a sufficiently large gap between the two-photon and one-photon (ionization) cross sections, it should be possible to choose laser pulse energy and focussing conditions such that these saturation requirements are met. This can be shown by examining expressions for the excited fraction in each process, $f=1-\exp ^{-W t}$, where $W$ is the transition rate, and $t$ is the laser pulse dura- 

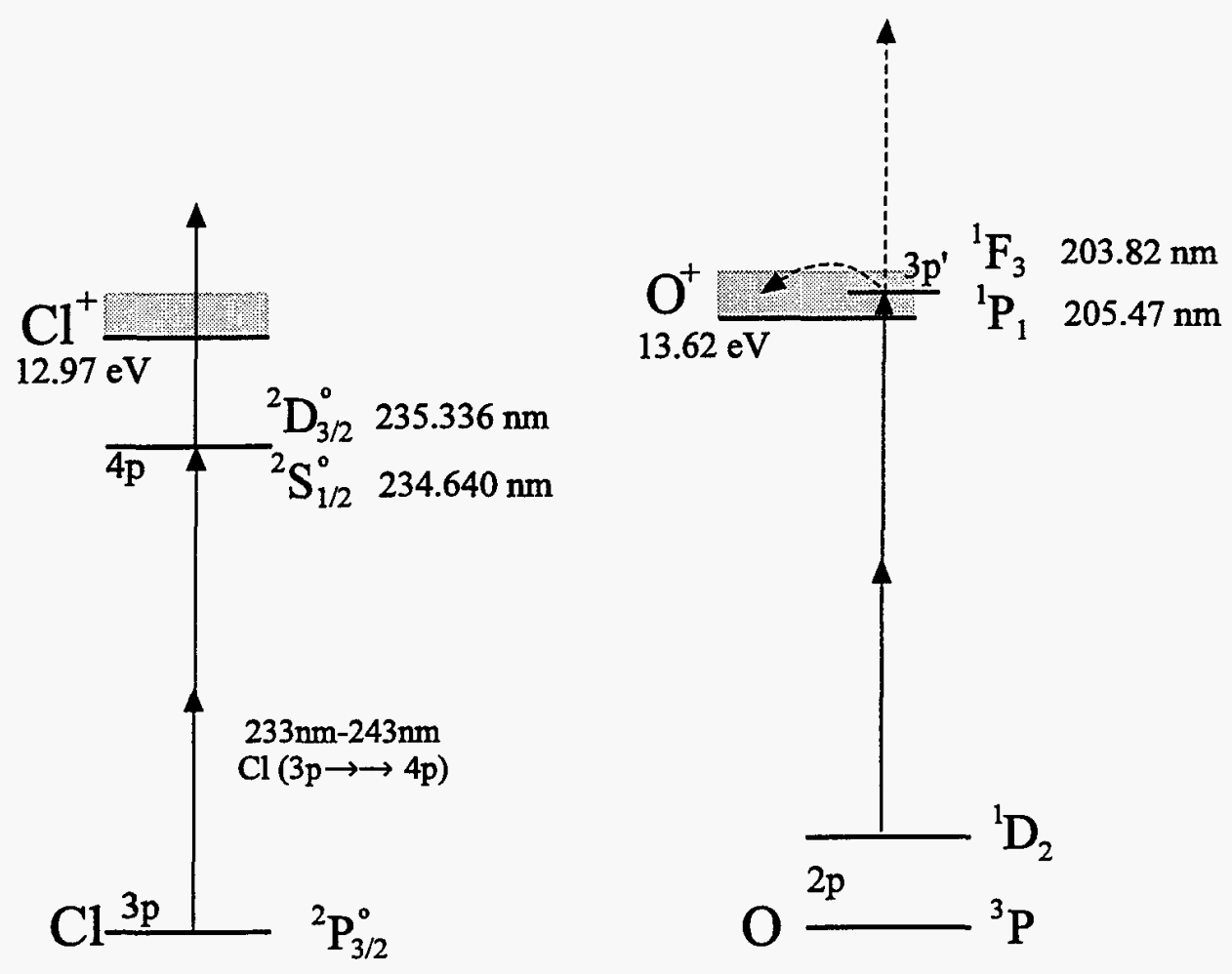

Figure 2.4: 2+1 REMPI schemes used in this work to detect chlorine [65] and oxygen [66] atomic photofragments. Note that the two-photon states for oxygen atoms may autoionize rather than absorb a third photon. 
tion. The expressions for $W \times t$ in terms of laser pulse energy, wavelength, absorption cross section, and spot radius are

$$
\begin{aligned}
& W_{2 p h} t=\sigma_{2 p h} F^{2} t \approx 1.6 \times 10^{48} \frac{[E(m J)]^{2} \times[\lambda(n m)]^{2} \times\left[\sigma_{2 p h}\left(\mathrm{~cm}^{4} s\right)\right]}{[r(\mu m)]^{4}} \\
& W_{1 p h} t=\sigma_{1 p h} I \approx 2.6 \times 10^{20} \frac{[E(m J)] \times[\lambda(n m)] \times\left[\sigma_{1 p h}\left(\mathrm{~cm}^{2}\right)\right]}{[r(\mu m)]^{2}}
\end{aligned}
$$

where $F$ is a photon flux (i.e. per unit area and time) and $I$ is a photon fluence (i.e. per unit area). The first expression above assumes a laser pulse with 8 ns duration. Fig. 2.5 shows the excited fraction $f$ for both one- and two-photon steps as a function of spot radius, for a REMPI laser pulse energy of $0.05 \mathrm{~mJ}$. Two hypothetical ionization cross sections are shown. The higher value allows a reasonably wide range over which the spot size can change, while still keeping the one- and two-photon steps saturated and not saturated, respectively. In other words, only a small fraction of atoms are excited, but nearly all of those excited are ionized. This region is indicated by the bar in the figure. For the lower ionization cross section, the desired saturation conditions cannot be met.

In general, while it should be possible to choose experimental conditions where saturation effects are avoided, the above discussion suggests that one must use caution in making these choices. If one works in a regime where saturation (or lack of it in the ionization step) do affect a polarization measurement, then the degree of saturation should be carefully characterized and included in the data analysis.

In order to study the $\mathbf{v}-\mathbf{J}$ correlation, it is necessary to change the relative polarizations of the dissociation and probe lasers. In imaging or TOFMS profile experiments, it is also desirable to change both laser polarizations with respect to the ion flight axis, in order to obtain two different projections of the same threedimensional ion distribution. Proper polarizations were confirmed by minimizing transmission through a UV Glan prism oriented to reject the desired polarization. For standard Nd:YAG photodissociation wavelengths, the rotations were achieved with an appropriate half wave plate. For the probe laser light, which was usually at wavelengths for which half-wave plates were not readily available, a wavelengthindependent double Fresnel rhomb was used. The double Fresnel rhomb has the 


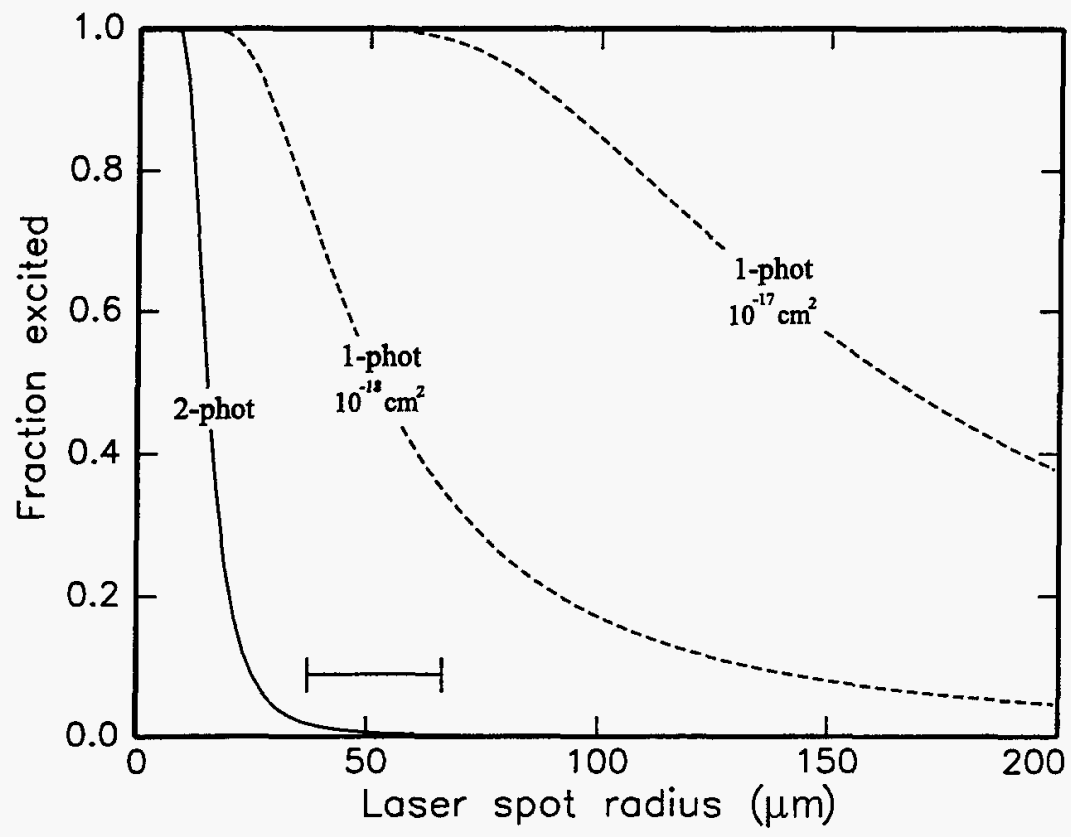

Figure 2.5: Saturation behavior of two-photon and one-photon (ionization) steps in $2+1$ REMPI process for chlorine atoms. The following values were used: $\lambda=235 \mathrm{~nm}$, $E=0.1 \mathrm{~mJ}, \sigma_{1 p h}=1 \times 10^{-17(-18)} \mathrm{cm}^{2}$, and $\sigma_{2 p h}=10^{-46} \mathrm{~cm}^{4} \mathrm{~s}$. The two hypothetical values for $\sigma_{1 p h}$ span the range of ionization cross sections (from the ground state) of similar atoms. Cross section $\sigma_{2 p h}$ was calculated by dividing a typical result from Refs [67, 68 ] by a linewidth of $10 \mathrm{GHz}$. The bar indicates the region of desirable saturation conditions (for the higher ionization cross section) as discussed in the text.

disadvantage that it is very difficult to align and rotate in such a way that the focussed probe beam does not translate slightly in the interaction region. Although slight beam realignments were straightforward, it was impossible to perfectly reproduce the spatial overlap, since the beam's spatial profile was also rotated. Slight image asymmetries usually result from imperfect overlap, and if these asymmetries are not consistent across data sets, they can be mistaken for the effects of vector correlations. Finally, at wavelengths below $250 \mathrm{~nm}$, the double Fresnel rhomb produces a small fraction of ellipticity in the $45^{\circ}$ (polarization rotated) position. In the region of the Cl REMPI transitions around $235 \mathrm{~nm}$, this contribution was roughly $10 \%$. More quantitative results will require the use of a Pockels cell, photoelastic modulator, or custom wave plates for this wavelength. For the oxygen atom REMPI at 203-205 nm, the double Fresnel rhomb was not used, and instead additional turns were added in the beam 
path to effect a polarization rotation.

\subsection{Ion Imaging and Time-of-Flight Mass Spec- trometry}

\subsubsection{Operating Principle and General Design Considera- tions}

The simplest time-of-flight mass spectrometer (TOFMS) consists of an acceleration (extraction) region, a flight path to allow mass separation, and a charged particle detector such as a channeltron or microchannel plate. In general, ionizationbased detection methods provide three great advantages over fluorescence techniques. First, the detection sensitivity is higher because charged particle detectors operate with high quantum efficiency and potential signal is not lost to the $4 \pi$ steradiancy of emission. Second, mass separation permits discrimination of the desired ions from non-resonantly-produced background ions. This is especially important when using a two-photon excitation scheme, since the required high power densities can easily fragment and ionize traces of oil vapor in the experimental apparatus. The third advantage is the ability to manipulate the shape of the ion packet, and thus also the mass or recoil energy resolution, by tuning the ion extraction fields in a two-stage extraction region. It has also been demonstrated recently that meshless einzel lens conditions can reduce lateral spatial blurring in imaging experiments [69, 70].

A very common and versatile design for TOFMS uses two-stage ion extraction optics, first discussed by Wiley and McClaren [71]. The ions are created in the first stage, and the electric field in this region serves to fine-tune the TOF peak shape for a given mass. The higher electric field in the second stage provides the bulk of the acceleration and sweeps the ions rapidly into the field-free flight tube. By adjusting the field in the first (low field) stage, one may 'focus' the ion packet in order to compensate for either spatial or kinetic energy spreads in the initial ion packet. (Focussing in this sense refers to modifying the flight-axis component of the ion packet, 
rather than lateral components.)

If there is only a single extraction stage, a wide initial ion packet would cause blurring of the TOF mass peaks [Fig. 2.6(b)]. The ions furthest back from the detector have higher potential energy (and final velocity) but a longer flight length, so that the two effects compensate only for a specific detector length. A second extraction stage provides an additional adjustable parameter so that the backmost ions may catch up, and all ions reach the detector [Fig. 2.6(b)] at the same time. This condition is described as 'space focussing'. 'Energy focussing' compensates for initial ion velocity (e.g. photoproduct recoil or space-charge effect) which would also blur the mass resolution. In this operating mode, the first stage remains field free for a short delay time as the recoil energy is allowed to slightly expand the ion packet. When the field is finally switched on, those ions which were flying backwards and would otherwise have appeared at the trailing edge of the TOF peak, are now able to catch up with the forward-recoiling ions, because they had flown to the region of higher potential energy during the field-free delay. In experiments where the ion packet has both a wide spatial extent and significant recoil energy, some compromise of the two modes will be necessary, since they cannot simultaneously function under maximumresolution conditions. For the core-sampling experiments described in Chapter 5, the energy focussing condition was deliberately detuned to improve resolution of the photofragment recoil velocities.

For spectroscopic applications of TOFMS, the specific shape of the ion packet is unimportant, since only the absolute intensity of the ion signal is measured. In this case, ion extraction optics can be built with simple electrostatic lenses (circular apertures) to separate the stages. In contrast, until recently, ion imaging measurements have used fine wire meshes between the extraction stages in order to maintain parallel field lines which minimize lateral distortions to the ion packet. The major sources of blurring in this design are the spatial extent of the initial ion packet (e.g. due to molecular beam width) and field distortions at the mesh. The latter can be reduced by using a finer mesh at the cost of lower transmission. In our experience, the most severe distortions occur at the mesh between the first and second stages, where the size of the ion packet is still comparable to the mesh spacing. To eliminate 
(a)

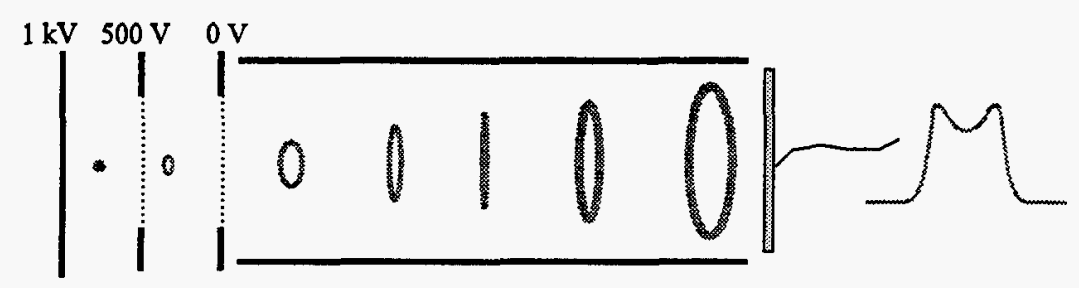

(b)

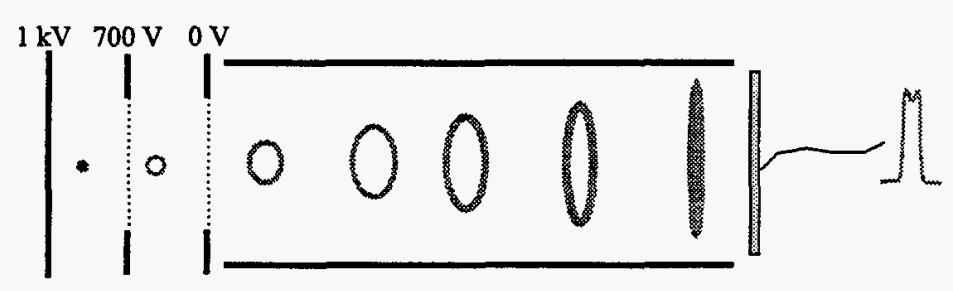

Figure 2.6: Operating principle of Wiley-McClaren 'space focussing' condition. (a) In general, the spatial extent of the initial ion packet will result in blurring of timeof-flight peaks. (b) If the electric fields in two extraction stages are independently adjustable, the full compression of the sphere can be timed to occur when it strikes the detector, leading to much sharper mass resolution.

this problem we have forgone the Wiley-McClaren capability by operating with equal electric fields in both stages. The resultant lowering of mass resolution is of little consequence for imaging experiments, since there are rarely interfering masses which cannot be discriminated with a pulsed microchannel plate detector.

The recent rediscovery of einzel lens extraction as so called 'velocity mapping' represents a profound improvement in the quality of ion imaging spatial resolution, providing velocity resolution more than an order of magnitude higher than that which is typically obtainable with meshes $[69,70]$. As of this writing (only several months since the technique was first discussed in conferences), numerous research groups have already adapted their old extraction optics for this method, since there no longer seems to be any advantage to using meshes in ion extraction optics. The technique relies on the well-known lateral focussing behavior of an einzel lens system to eliminate contributions to image blurring which result from the spatial extent of the ion packet. Naturally, mesh distortions are also eliminated. 


\subsubsection{Extraction Optics and Position-Sensitive Detector}

A convenient design for mounting the extraction meshes is shown in Fig. 2.7. Each mesh is sandwiched between two metal rings, one of which contains a thin o-ring in a slanted groove. When the rings are pressed together, the o-ring stretches outward as it settles into the groove, pulling the mesh tight and flat. For the experiments described in this thesis, nickel mesh with roughly 100 lines per inch was used. The metal rings were roughly three inches in diameter and spaced $1 \mathrm{~cm}$ apart. With these dimensions, additional empty rings between the main mesh-mounting rings are not necessary to obtain parallel field lines near the detector centerline axis. New editions of this design which are adapted for the velocity-mapping technique are much simpler. No mesh is needed, and simple round plates with 1-2 cm centered holes are sufficient. However, the design for overall assembly is still recommended for its convenience: hollow ceramic (or vespel) cylinders are used as spacers between the rings, and the entire assembly is pulled together with nylon threaded rods which pass through the vespel spacers and the rings. This three-plate unit is attached as a whole to the flight tube.

The position-sensitive detectors used in our imaging experiments consist of chevron double microchannel plates (MCP) in front of a phosphor screen. These items are usually mounted in a metal flange with a window or fiber-optic bundle to permit viewing of the phosphor from outside the vacuum. The detector assemblies used in our group were purchased commercially, although some groups have assembled their own. Typically the MCP is operated with a large negative voltage $(1.5-3 \mathrm{kV})$ on its front (where the ions strike) and near-neutral voltage in back. With these settings, it is necessary to shield the MCP in front with a fine mesh at a distance of a few millimeters in order to avoid field penetration into the flight tube. Any lateral field distortion near this mesh is of no consequence since the detector is so close. In the mass-spectrometer mode, the detector is run with continuous (DC) voltages, but for mass discrimination, the $\mathrm{DC}$ voltage difference across the $\mathrm{MCP}$ is reduced by several hundred volts, and a positive pulse ( $\sim 200 \mathrm{~ns})$ is applied to the back of the MCP to provide additional gain during the arrival time of the desired mass. 

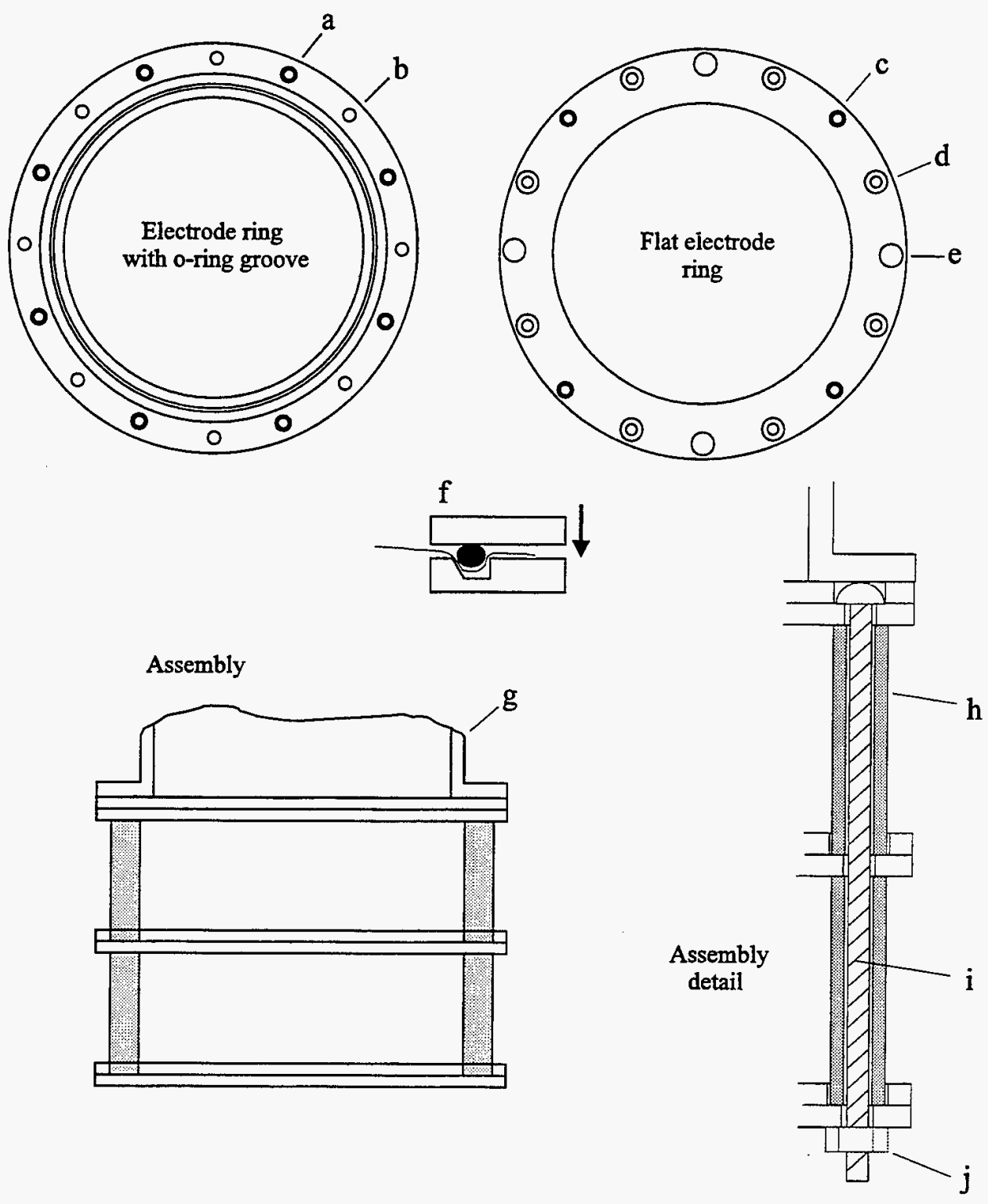

Figure 2.7: Ion extraction optics design: (a) Tapped hole for clamping oring. (b) Clearance hole for nylon assembly bolts, (c) Tapped holes for mounting to flight tube (unused in lower electrodes), (d) Countersunk clearance hole for clamping oring, (e) Minimal clearance hole for support posts, (f) Cross section of oring clamping action, (g) Flight tube, (h) Insulating support posts (hollow cylinders), (i) Roundhead nylon bolt, (j) nut. 
The phosphor screen is positioned roughly $2 \mathrm{~mm}$ behind the MCP and biased at 3-5 kV. Electron showers emitted from each MCP channel are accelerated and strike the phosphor, yielding spots of roughly $100 \mu \mathrm{m}$ diameter which are easily visible to the naked eye. The two most common phosphor types are P47 (blue) and P20 (green). Both have been used in our laboratory. The advantage of blue phosphors is their short lifetime, which is only tens of nanoseconds. Green phosphors have near-microsecond lifetimes, which are inconvenient for measuring mass spectra. Although CCD video cameras are considerably less sensitive to the blue phosphors, in our experience this has not been a significant limitation.

Image acquisition hardware for a typical imaging experiment consists of a video camera and a 'frame-grabber', which is interfaced to a computer and may have fast image processing capabilities. A common problem with CCD (charge-coupled device) video cameras is dark noise. Although individual ion spots are bright, over long data acquisition periods the continuously-accumulating camera noise becomes significant. Three approaches to this problem have been tried in our group. Some camera systems (e.g. Princeton Instruments) are water- or liquid nitrogen-cooled to reduce such noise. A different option is to use a simpler camera with more sophisticated frame-grabber hardware which can execute a 'clip' function at every laser shot to remove signal below a certain threshold level. Even fancier (and more expensive) systems can do two-dimensional ion counting at every shot.

\subsubsection{Treatment of Raw Image Data}

A sample of raw image data from chlorine dissociation is shown on the top of Fig. 2.8. In the absence of any experimental imperfections, one would expect these images to be symmetric under reflection through a horizontal or vertical axis. Although it not strongly pronounced in the black-on-white colormap used for this figure, the raw images are actually not symmetric-there is variation of up to $20 \%$ between opposing points on the image which should otherwise be equal. It is a common practice in ion imaging to symmetrize the raw data images by averaging together the original image with its $X, Y$, and $X / Y$ reflections. This amounts to an average of 


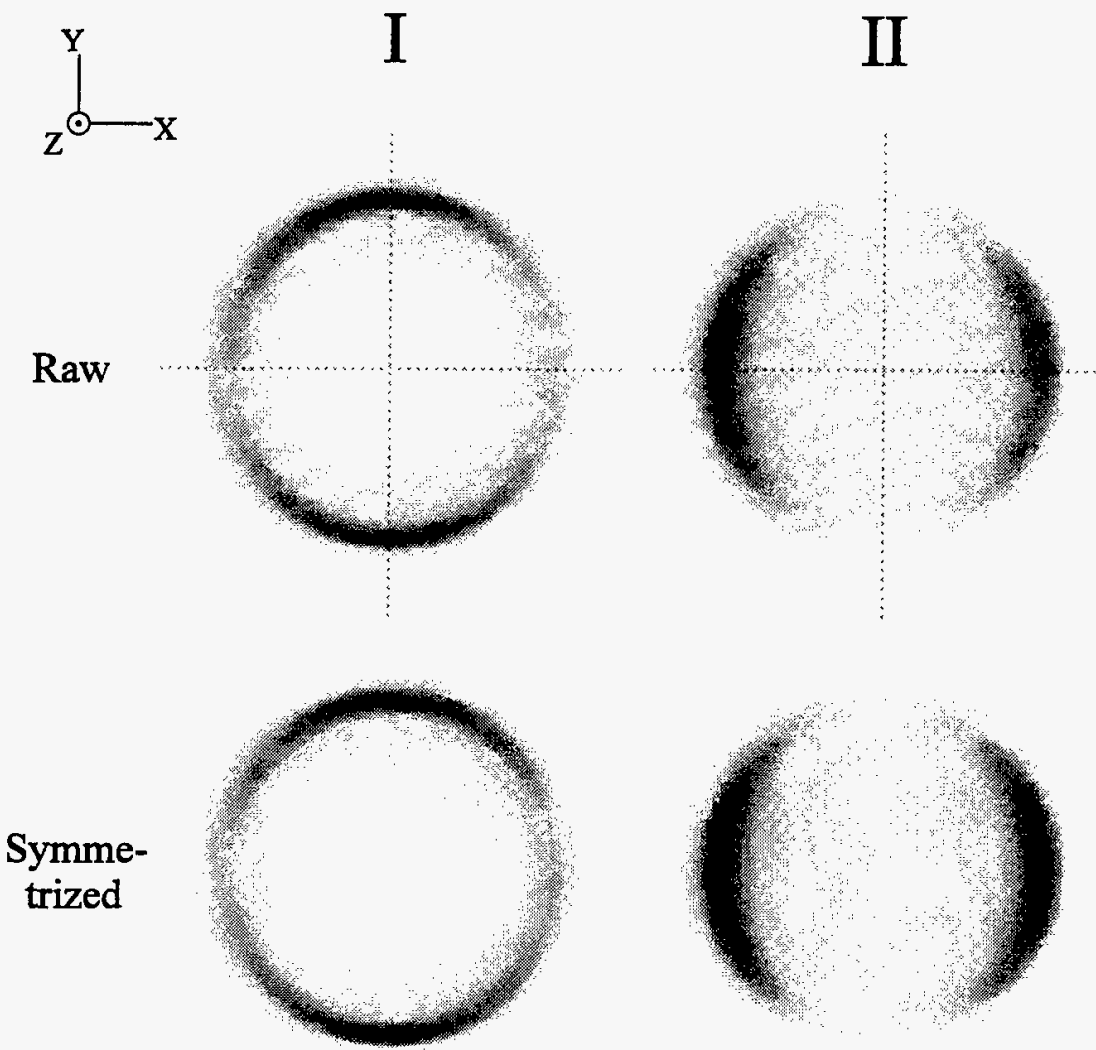

Figure 2.8: Sample raw and symmetrized data images for chlorine dissociation at $355 \mathrm{~nm}$. Symmetrized images are obtained by averaging the original image with its $X, Y$ and $X / Y$ reflections.

four independent measurements on different regions of the $\mathrm{MCP} /$ phosphor imaging detector. Because of inhomogeneities across the detector surface, these measurements are all slightly different. We can calculate a standard deviation associated with the averaging of the four measurements. An example of this for Geometry II is shown in the form of an $X Y Z$ wire mesh plot in Fig. 2.9. The standard deviations are roughly $10 \%$ of the total signal size. This problem of uneven spatial sensitivity is inherent to imaging. We have considered some techniques to eliminate these spatial problems, including calibrations based on a known spatial distribution, or further 'averaging out' of the inhomogeneity by rotating the detector through a range of angles with some sort of moveable flange.

A second feature to note about the images in Fig. 2.8, specifically for the 


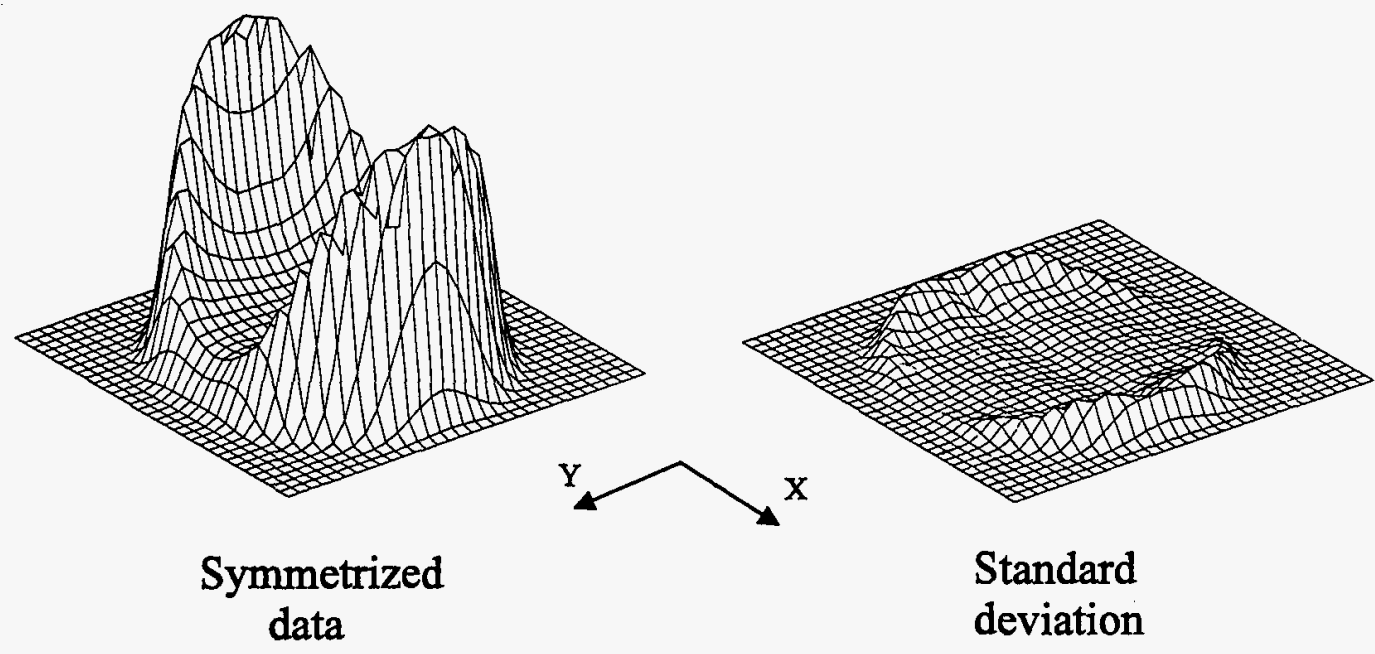

Figure 2.9: Symmetrized data image (Geometry II here) and standard deviation associated with the symmetrization.

Geometry I images, is the difference in signal intensity along the $X$ axis compared to the $Y$ axis. Since these images were collected with both dissociation and probe laser polarizations perpendicular to the image plane, we would expect them to be circularly symmetric. The observed variation is a consequence of the difference in spatial resolution in the $X$ and $Y$ directions. The resolution in the $X$ direction is limited by the size of the initial ion packet, which is determined by the width of the molecular beam (1-2 mm). In the $Y$ dimension, the ion packet is only as wide as the laser beam $(<400 \mu \mathrm{m})$, and the spatial resolution is governed mostly by the distorted electric fields at the mesh. Of course, the presence of a $\mathbf{v}-\mathbf{J}$ correlation will also produce an intensity modulation around this circle if the probe laser polarization is not perpendicular to the image plane. In order to separate the two effects, we calculate 'difference images', which isolate the effects of angular momentum alignment. This procedure is discussed in detail in Chapters 3 and 4.

\section{$2.4 \vec{v}-\vec{\jmath}$ Correlation Measurements}

As already discussed, measurement of a $\mathbf{v}-\mathbf{J}$ correlation demands simultaneous determination of the velocity and angular momentum vector distributions. This 
section provides a qualitative description of the measurement principle and defines the experimental geometries which are used in a typical ion imaging experiment.

The combined measurement is easily visualized as follows. A collection of molecules is photodissociated, creating a point source of recoiling photoproducts. At some time delay, each recoil energy corresponds to a spherical shell of fragments with a fixed radius. The neutral photofragments are distributed around these expanding spheres according to the usual photofragment angular distribution [Eq. (1.1)]. For standard Doppler, TOFMS, and imaging methods, the neutral fragment ensemble is probed by REMPI before leaving the initial photolysis volume. If a $\mathbf{v}-\mathbf{J}$ correlation exists, the resulting ion spheres will have a further angular modulation due to the sensitivity of the probe to angular momentum polarization. In ion imaging, one measures a 2-dimensional projection of this ion distribution, while Doppler and TOFMS profiles are 1-dimensional projections. In some 'differential' techniques, only a subset of recoil angles is probed. This sampling can be done by the laser on an expanded neutral sphere $[19,20,72]$, by a mechanical aperture or slit on the expanded ion sphere $[16,57]$ or with a combination of both [17].

In order to emphasize and isolate the angular modulations in the ion spheres that are caused by the $\mathbf{v - J}$ correlation, one compares data collected under different probe-laser conditions. One may look for changes in the image upon rotation of the probe polarization (in a two laser experiment) or upon choosing a different probe transition. As mentioned in Section 2.2.1, these actions correspond to measuring different projections of a transition moment ensemble and to choosing a new ensemble of transition moments, respectively. Fig. 2.10 provides a classical depiction of a $\mathbf{v}-\mathbf{J}$ correlation measurement for the limiting case of $\mathbf{J}$ strictly parallel to $\mathbf{v}$. (The example is classical in the sense that $\mathbf{J}$ is sharply-peaked.)

If we choose a 'parallel' probe transition $(\Delta \mathrm{J}=0, \boldsymbol{\mu} \| \mathbf{J})$, we have $\boldsymbol{\mu} \| \mathbf{v}$. Probing with vertical linearly polarized light, as shown in Fig. 2.10(b), will selectively ionize those fragments flying out with a velocity component parallel to the probe polarization. Of course, rotating the probe polarization rotates the distribution of lab-frame ionization probability as well-a characteristic which serves as a common signature for a $\mathbf{v}-\mathbf{J}$ correlation. On the other hand, if we choose a 'perpendicular' 
(a)
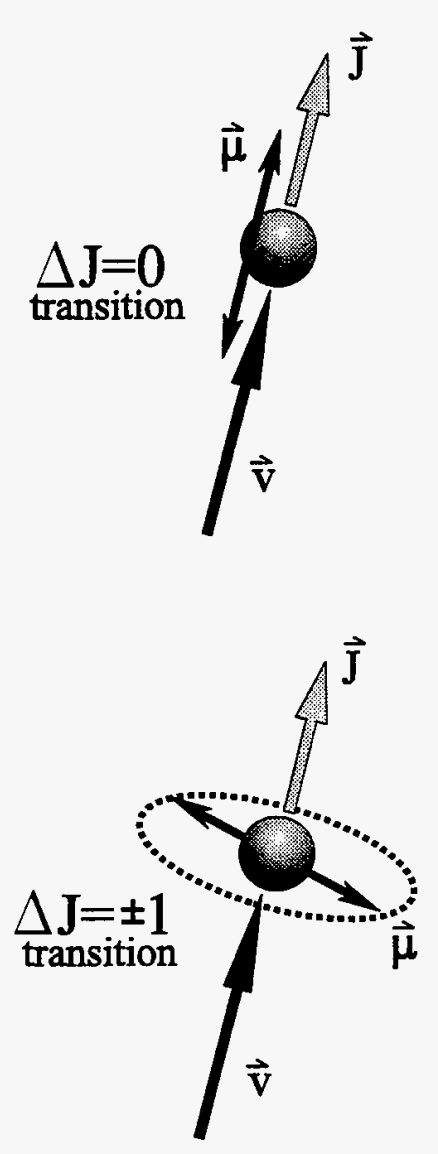

(b)
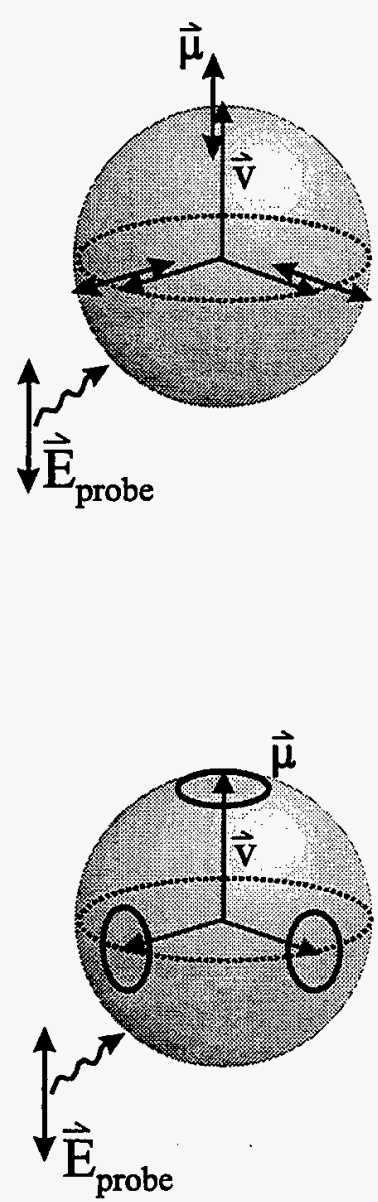

(c)
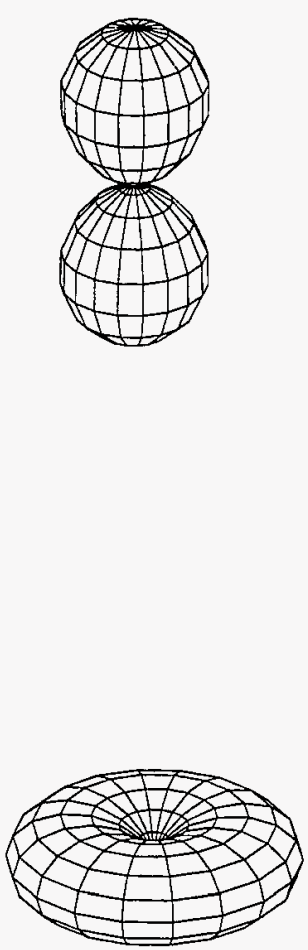

Figure 2.10: Schematic of $\mathbf{v}-\mathbf{J}$ correlation measurement principle, for classical limiting case $\mathbf{v} \| \mathbf{J}$. If a $\mathbf{v}-\mathbf{J}$ correlation exists, the shape or orientation of the resulting ion distribution will change if the REMPI probe transition or laser polarization is changed, respectively. (a) Relative orientation of relevant vectors for two types of transitions. (b) A spherical shell of photofragments (isotropic for simplicity) probed by vertically polarized laser. The transition moment $\boldsymbol{\mu}$ is shown for three orthogonal velocity vectors. (c) Angular distribution of ionization probability (= angular distribution of ions for isotropic distribution of neutrals). Projections of this distribution are measured with ion imaging (two dimensions) or as TOFMS profiles (one dimension). 


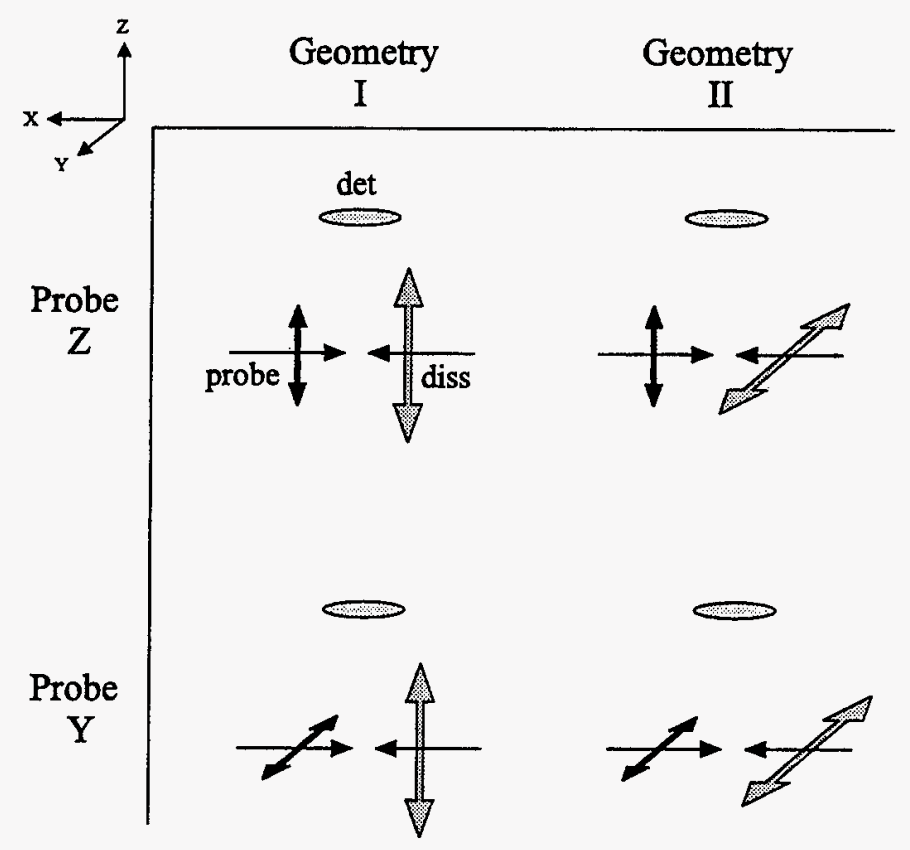

Figure 2.11: Experimental geometries used in this work. Geometries I and II refer to the dissociation laser polarization along the $Z$ and $Y$ axes, respectively. The probe laser polarization is also along either the $Z$ or $Y$ axis, though no special label is given.

probe transition $(\Delta \mathrm{J}= \pm 1, \boldsymbol{\mu} \perp \mathbf{J})$, we have $\boldsymbol{\mu} \perp \mathbf{v}$ and we selectively ionize the product fragments which fly out with a velocity component perpendicular to the probe polarization. For diatomic photofragments, the two choices of probe transition would correspond to a $\mathrm{Q}$ branch line and $\mathrm{P} / \mathrm{R}$ branch line, respectively.

In a typical imaging study of $\mathbf{v}-\mathbf{J}$ correlations, four different relative configurations of the dissociation and probe laser polarizations and the detector axis are used. These configurations are shown in Fig. 2.11. In practice, the modulations in ionization probability which concern us for atoms are not as pronounced as those depicted in Fig. 2.10(c), since the uncertainty principle prohibits sharply peaked angular momenta when $J$ is small. As described in more detail in Chapter 3, it is useful to isolate the contribution to the signal which is due solely to the $\mathbf{v}-\mathbf{J}$ correlation by combining images collected with different probe laser conditions. In a conventional experiment with linearly polarized light, one collects data for two positions of the probe laser polarization, corresponding to $Z$ and $Y$ with our axis definitions [Fig. 2.1]. 
In an imaging experiment, it is also desirable to collect data for two perpendicular dissociation laser polarizations, also along $Z$ and $Y$. Throughout the remainder of this work, these two dissociation geometries are referred to as Geometry I and Geometry II, respectively. Measurements in these geometries provide two different projections of the three-dimensional distribution of ions. For systems with a single recoil velocity (e.g. chlorine dissociation), the results from one dissociation geometry contain all the desired information, however, data from a second Geometry will improve measurement statistics and may permit one to isolate measurement artifacts from meaningful structure in the data. For polyatomic systems with a wide velocity distribution and a $\mathbf{v}-\mathbf{J}$ correlation which may depend on velocity, multiple dissociation geometries increase the possibility of obtaining a unique fit to the data. Also, as discussed in relation to Eq. (3.17), Geometry I has some advantages for isolating the signal modulations from $\mathbf{v}-\mathbf{J}$ correlations. This is in contrast to many imaging measurements, in which Geometry II is preferred since it permits one to apply the inverse-Abel transform [see Sec. 3.5] to pixel rows of ion image data. 


\section{Chapter 3}

\section{Data Analysis Method}

\subsection{Overview}

This chapter describes a general method for extraction and interpretation of angular momentum polarization information from ion image data. The end goal of such an analysis is to obtain a set of polarization anisotropy parameters which provide insight into the dissociation mechanism. These parameters are analogous to the familiar parameter $\beta$ for the velocity anisotropy, except that they describe the angular distribution of angular momentum polarization instead of photofragment density. They are closely related to scattering amplitudes and thus provide a convenient meeting point between experiment and theory. The method described here will be used in the next chapter to interpret the experimental measurements on chlorine dissociation.

A simulation of ion image data consists of a linear combination of 'image basis functions', with the polarization anisotropy parameters (or simple combinations) as coefficients. Derivation of these basis functions consists of three steps. The starting point is a general expression for recoil-angle-dependent state multipoles, $\rho_{k q}(\phi, \theta)$. Specific instances of this expression must be evaluated for the desired experimental geometries, i.e. the dissociation laser polarization and the ion flight axis of the detector. Next, these state multipoles are integrated over the flight axis dimension in order to convert from spherical polar recoil coordinates to two-dimensional polar 
image coordinates. Finally, they are inserted into expressions for the two-photon probe transition probability. The transition probabilities are evaluated for different polarizations of the probe laser and then combined in such a way as to isolate the modulations in the signal that are due exclusively to angular momentum polarization.

A number of theoretical results are presented in this chapter. While the emphasis of this dissertation is on the analysis and interpretation of experimental results, it is impossible to tell a complete story without discussing specific theoretical expressions. Many of them have been printed elsewhere in the literature, and several expressions which relate to REMPI-imaging experiments are the direct output of our recent collaboration with Professor Oleg Vasyutinskii from the Ioffe Physicotechnical Institute in St. Petersburg. All of the expressions included here are central to an analysis of atomic photofragment polarization and should be understood by experimentalists in the field. They were either used directly in our own analysis of the data, or they provide valuable conceptual insight into the relevant physics. The approach of this chapter will be to discuss general conceptual insights as the formulas are presented, so that their specific application to the experimental data will require only a few additional comments to aid in understanding.

\subsection{Recoil-angle-dependent State Multipoles}

Angular momentum polarization can be conveniently described in terms of irreducible density matrix components (state multipoles), $\rho_{k q}[73,74]$. The state multipoles are related to the more familiar alignment parameters $\left(A_{k q}\right)$ by a $J$-dependent proportionality constant. In order to develop a convenient intuition about their meaning, it is useful to consider the state multipoles in the high- $J$ limit, where they correspond to the coefficients of a spherical harmonic expansion of the distribution $P\left(\omega_{j}\right)$. State multipoles with nonzero even values of the rank $k$ describe alignment, while those with odd $k$ correspond to orientation. The state multipole $\rho_{00}$ is proportional to population and serves as a normalization factor. The corresponding basis functions in the classical expansion are the $s, d, g \ldots$ and $p, f, h \ldots$ orbital shapes, respectively. The projection $q$ distinguishes, for example, $p_{x}, p_{y}$ and $p_{z}$, although the $\rho_{k q}$ 's are usu- 
ally expressed in a spherical rather than Cartesian basis and thus have both a real and a complex part.

As discussed in Chapter 1 , when we measure the $\mathbf{v - J}$ correlation, we are concerned with the distribution $P\left(\omega_{v}, \omega_{j}\right)$, so the state multipoles must be a function of recoil angle, i.e. $\rho_{k q}=\rho_{k q}\left(\omega_{v}\right) \equiv \rho_{k q}(\theta, \phi)$. In the field of molecular dissociation dynamics, Dixon provided the first such description [38] in terms of bipolar moments. This semiclassical approach has been useful for a variety of vector correlation studies involving diatomic photofragments, since it directly provides the geometric relationships between the vectors $\mathbf{v}, \mathbf{J}$ and $\boldsymbol{\mu}$. This sort of information is readily interpreted in terms of nuclear dynamics on a potential energy surface (see e.g. $[38,75,76]$ ). In principle, the same method can be adapted for atomic angular momenta, although it is not as convenient. The atomic angular momenta produced in dissociations are usually quite low, and quantum effects are important. The limiting-case correlations (e.g. $\mathbf{v} \perp \mathbf{J}$ and $\mathbf{v} \| \mathbf{J}$ ), which are a useful intuitive aid for understanding nuclear dynamics, are unrealistic for low $J$ due to the uncertainty principle. More importantly, simply knowing the geometric correlations for atomic angular momentum does not lead easily to a dynamical interpretation as it does for diatomic fragments.

The approach of Siebbeles et al. [41] is well-suited for atomic photofragments. It is a fully quantum-mechanical method which provides a direct relationship between angular momentum polarization and scattering amplitudes, allowing for more straightforward comparison of measurements with theory. This method allows an immediate mechanistic interpretation of angular momentum polarization, via the 'dynamical functions', $f_{k}\left(q, q^{\prime}\right)$, in terms of excited state symmetries and coherence effects. The central result of Ref. [41] is a general expression for the angle dependence of the state multipoles and is the starting point for the data analysis described in this thesis:

$$
\begin{aligned}
& \rho_{k q}(\theta, \phi)=\frac{2 \pi^{3 / 2} \omega}{c} \sum_{q q^{\prime}} \sum_{P V}(-1)^{q} \sqrt{2 P+1} \sqrt{2 V+1} \\
& \times\left(\begin{array}{ccc}
1 & 1 & P \\
\mathrm{q}^{\prime} & -\mathrm{q} & \mathrm{q}-\mathrm{q}^{\prime}
\end{array}\right)\left(\begin{array}{ccc}
P & V & k \\
\mathrm{q}^{\prime}-\mathrm{q} & 0 & \mathrm{q}-\mathrm{q}^{\prime}
\end{array}\right) \\
& \times\left[Y_{V} \otimes E_{P}\right]_{k q} f_{k}\left(\mathrm{q}, \mathrm{q}^{\prime}\right) \text {, }
\end{aligned}
$$


where the (:::) are Wigner $3-j$ symbols. The indices $P, V$ and $k$ are the tensor ranks which correspond to the dissociation photon, recoil velocity and angular momentum, respectively. ${ }^{1}$ The corresponding tensor projections are $p, v$ and $q$. The angular dependence of Eq. (3.1) is contained in the tensor product,

$$
\left[Y_{V} \otimes E_{P}\right]_{k q}=\sum_{p v}(-1)^{k_{2}-P+q} \sqrt{2 k+1} \times\left(\begin{array}{ccc}
V & P & k \\
v & p & -q
\end{array}\right) Y_{V v}(\theta, \phi) E_{P p}
$$

where $Y_{V v}(\theta, \phi)$ is a spherical harmonic. $E_{P p}$ is the tensor form of the density matrix for the photodissociation light. It is defined as follows, in terms of the components $e_{\xi}$ (in the spherical basis) of the light polarization vector e:

$$
E_{P_{p}}=\sqrt{2 P+1} \sum_{\xi \xi^{\prime}}(-1)^{\xi} e_{\xi} e_{\xi^{\prime}}^{*}\left(\begin{array}{ccc}
1 & 1 & P \\
\xi & \xi^{\prime} & -p
\end{array}\right) .
$$

Note that $P$ can be as high as two, even though the light polarization corresponds to a rank 1 tensor (i.e. vector), since these expressions originate from the square of the transition dipole matrix element for excitation of the parent molecule. The spherical basis components of $\mathbf{e}$ in terms of Cartesian components are $e_{ \pm 1}=\mp \frac{1}{\sqrt{2}}\left(e_{x} \pm i e_{y}\right)$ and $e_{0}=e_{z}$.

All information in Eq. (3.1) that relates to dissociation dynamics is contained within the dynamical functions $f_{k}\left(\mathrm{q}, \mathrm{q}^{\prime}\right)$. The indices $\mathrm{q}$ and $\mathrm{q}^{\prime}$ describe the spherical basis projections of the transition moment with respect to the internuclear axis and have the values 0 and \pm 1 for parallel and perpendicular transitions, respectively. ${ }^{2}$ Functions $f_{k}\left(\mathrm{q}, \mathrm{q}^{\prime}\right)$ with $\mathrm{q}=\mathrm{q}^{\prime}$ correspond to incoherent excitations, while those with $\mathrm{q} \neq \mathrm{q}^{\prime}$ represent coherent excitation. Because of the way that they appear in the $3-j$ symbols of Eq. (3.1), each possible combination of $\mathrm{q}$ and $\mathrm{q}^{\prime}$ in $f_{k}\left(\mathrm{q}, \mathrm{q}^{\prime}\right)$ will lead to a unique angular dependence for $\rho_{k q}(\theta, \phi)$ (see footnote ${ }^{3}$ ). This fact demonstrates

\footnotetext{
${ }^{1}$ The same indices appear in the bipolar moments method in a different notation. The correspondence is $(P, V, k) \rightarrow\left(K, k_{1}, k_{2}\right)$ and $(p, v, q) \rightarrow\left(Q, q_{1}, q_{2}\right)$, where the symbol for a bipolar moment is usually written $b_{Q}^{K}\left(k_{1} k_{2}\right)$.

${ }^{2}$ Indices $\mathrm{q}$ and $\mathrm{q}^{\prime}$ are distinct from italic $q$, which is the projection index for the state multipoles $\rho_{k q}(\theta, \phi)$. When context is considered, this usage is not as confusing as it may first appear. This and several other notational compromises were made to include expressions from different sources.

${ }^{3}$ Not all $f_{k}\left(\mathbf{q}, \mathbf{q}^{\prime}\right)$ are independent, however, since they must obey the symmetry relations $f_{k}\left(\mathbf{q}, \mathrm{q}^{\prime}\right)$ $=(-1)^{k} f_{k}\left(-\mathrm{q},-\mathrm{q}^{\prime}\right)=(-1)^{\mathrm{q}-\mathrm{q}^{\prime}} f_{k}^{*}\left(\mathrm{q}^{\prime}, \mathrm{q}\right)$
} 
mathematically why it is advantageous to study $\mathbf{v}-\mathbf{J}$ correlations instead of the angleintegrated polarization. Further qualitative insights that are apparent from Eq. (3.1) are discussed in the following section, while Sec. 4.4 addresses the detailed form of the dynamical functions.

\subsection{Polarization Anisotropy Parameters and Spe- cific Expressions for $\rho_{k q}(\theta, \phi)$}

The first step in making use of Eq. (3.1) is to evaluate it for the specific geometries employed in the experiment. In a REMPI-imaging experiment, we are concerned with the relative orientation of the dissociation laser polarization, the detector flight axis and the probe laser polarization. In this section we consider only the first two, leaving the probe laser geometry for the next section. Since our experimental data consist of pixels in the $X-Y$ plane of the detector, it is most natural to express the state multipoles (and later the REMPI intensity) in a frame specified by the detector axis, $\mathbf{Z}=\mathbf{Z}_{\text {det }}$. As discussed earlier, two dissociation laser geometries were used: Geometry I has $\boldsymbol{e}_{\text {diss }} \| \mathbf{Z}$ while Geometry II has $\boldsymbol{e}_{\text {diss }} \| \mathbf{Y}$. The tensor nature of Eq. (3.1) makes it very easy to evaluate for these experimental geometries, by using the polarization tensor components

$$
\begin{array}{lll}
\text { Geometry I: } & E_{00}=1 / \sqrt{3} & E_{20}=-2 / \sqrt{6} \\
\text { Geometry II: } & E_{00}=1 / \sqrt{3} \quad E_{20}=1 / \sqrt{6} \quad E_{2 \pm 2}=1 / 2 .
\end{array}
$$

These values were obtained from Eq. (3.3) by using $\mathbf{e}=e_{z} \mathbf{Z}$ and $\mathbf{e}=e_{y} \mathbf{Y}$ for Geometries I and II, respectively. Evaluation of Eq. (3.1) for these geometries yields the necessary expressions for $\rho_{k q}(\theta, \phi)$ in terms of dynamical functions.

At this stage, it is desirable to rewrite the expressions for $\rho_{k q}(\theta, \phi)$ in terms of 'polarization anisotropy parameters' [77], which are convenient normalized combinations of the dynamical functions. Since the absolute size of the dynamical functions is not readily apparent, we divide by the total cross section in order to normalize them. The total cross section is proportional to the sum over all three transition mo- 
ment projections of the $k=0$ dynamical functions, i.e. $f_{0}(0,0)+f_{0}(1,1)+f_{0}(-1,-1)$ or equivalently, $f_{0}(0,0)+2 f_{0}(1,1)$. Also, the expressions become simpler when dynamical functions which multiply the same $(\theta, \phi)$-dependence are grouped together. The resulting anisotropy parameters that are relevant for alignment are:

$$
\begin{aligned}
s_{2} & =V(j)^{-1} \frac{f_{2}(0,0)+2 f_{2}(1,1)}{f_{0}(0,0)+2 f_{0}(1,1)} \\
\alpha_{2} & =V(j)^{-1} \frac{f_{2}(1,1)-f_{2}(0,0)}{f_{0}(0,0)+2 f_{0}(1,1)} \\
\gamma_{2} & =2 \sqrt{3} V(j)^{-1} \frac{\operatorname{Re}\left[f_{2}(1,0)\right]}{f_{0}(0,0)+2 f_{0}(1,1)} \\
\eta_{2} & =\sqrt{6} V(j)^{-1} \frac{f_{2}(1,-1)}{f_{0}(0,0)+2 f_{0}(1,1)},
\end{aligned}
$$

where $V(j)=5\{j(j+1) /[(2 j+3)(2 j-1)]\}^{1 / 2}$. These parameters each represent a contribution to the total alignment parameter $A_{20}$ from different excitation mechanisms. (By integrating $\rho_{20}(\theta, \phi)$ over recoil angles, it can be shown that $A_{20} \propto \alpha_{2}+\gamma_{2}+\eta_{2}$.) Parameters $s_{2}$ and $\alpha_{2}$ describe alignment due to incoherent excitation $\left(q=q^{\prime}\right)$, via both parallel and perpendicular transitions. Parameter $\gamma_{2}$ corresponds to simultaneous parallel $(q=0)$ and perpendicular $\left(q^{\prime}= \pm 1\right)$ excitation. This parameter will be important when two electronic states with electronic projections $\Omega$ equal to \pm 1 and 0 have comparable excitation probabilities. Parameter $\eta_{2}$ describes coherent perpendicular transitions. This may occur for two states slightly separated in energy as in the parallel/perpendicular case. A more common possibility is simultaneous excitation of the degenerate $\Omega= \pm 1$ 'lambda doublet' components of an $|\Omega|=1$ state, since both states automatically have equal excitation probabilities.

For the chlorine study, we need expressions only for the state multipoles $\rho_{00}(\theta, \phi), \rho_{20}(\theta, \phi)$, and for the real portion of $\rho_{22}(\theta, \phi)$. The $j=3 / 2$ atoms can only have $k \leq 2$, while the probe geometries that we have chosen restrict which alignment projections $q$ are needed. The latter restrictions are discussed briefly in Section 3.4. Using the above definitions of the alignment anisotropy parameters yields the following expressions for the state multipoles: 
Geometry I

$$
\begin{aligned}
& \rho_{00}(\theta, \phi)=\sigma_{0}\left[1+\beta_{0}^{\prime} P_{2}(\cos \theta)\right] \\
& \rho_{20}(\theta, \phi)=\sigma_{0}\left\{P_{2}(\cos \theta)\left[s_{2}-2 \alpha_{2} P_{2}(\cos \theta)\right]-3 \gamma_{2} \sin ^{2} \theta \cos ^{2} \theta-\frac{3}{4} \eta_{2} \sin ^{4} \theta\right\} \\
& \rho_{22}(\theta, \phi)=\sqrt{5} \sigma_{0} \sin ^{2} \theta e^{2 i \phi}\left\{\left[s_{2}-2 \alpha_{2} P_{2}(\cos \theta)\right]+2 \gamma_{2} \cos ^{2} \theta-\frac{1}{2} \eta_{2}\left(1+\cos ^{2} \theta\right)\right\}
\end{aligned}
$$

Geometry II

$$
\begin{aligned}
\rho_{00}(\theta, \phi)= & \sigma_{0}\left[1+\beta_{0}\left(1-3 \sin ^{2} \theta \sin ^{2} \phi\right)\right] \\
\rho_{20}(\theta, \phi)= & \sigma_{0}\left\{P_{2}(\cos \theta)\left[s_{2}+\alpha_{2}\left[P_{2}(\cos \theta)(1-\cos 2 \phi)+\cos 2 \phi\right]\right]\right. \\
& +\frac{3}{2} \gamma_{2} \sin ^{2} \theta \cos ^{2} \theta(1-\cos 2 \phi) \\
& \left.+\frac{3}{8} \eta_{2} \sin ^{2} \theta\left[\sin ^{2} \theta+\left(1+\cos ^{2} \theta\right) \cos 2 \phi\right]\right\} \\
\rho_{22}(\theta, \phi)= & \sigma_{0} e^{2 i \phi}\left\{\sin ^{2} \theta\left[s_{2}+\alpha_{2}\left[P_{2}(\cos \theta)(1-\cos 2 \phi)+\cos 2 \phi\right]\right]\right. \\
& +\gamma_{2} \sin ^{2} \theta\left[\cos ^{2} \theta(1-\cos 2 \phi)+i \sin 2 \phi\right] \\
& \left.-\frac{\eta_{2}}{4}\left[\left(1+\cos ^{2} \theta\right) \sin ^{2} \theta+\left(1+\cos ^{2} \theta\right)^{2} \cos ^{2} \phi-4 i \cos ^{2} \theta \sin ^{2} \phi\right]\right\} .
\end{aligned}
$$

The parameters $\beta_{0}^{\prime}$ and $\beta_{0}[41,77]$ are the usual velocity anisotropy parameter $\beta$ for $Z$ - and $Y$-polarized light, respectively.

Although it is apparent from Eqs (3.6) and (3.7) that each of the alignment anisotropy parameters is associated with a unique contribution to the angular dependence, the physical basis for these dependencies is more transparent when alignment in the molecular recoil frame is considered. A general expression for the molecular frame state multipoles appears in the paper of Siebbeles et al. [41] but will not be shown here. The specific expressions for the $k=0,2$ multipoles for linearly polarized light along the $Z$-axis are

$$
\begin{aligned}
& \rho_{00}(\theta, \phi)=\sigma_{0}\left[1+\beta_{0}^{\prime} P_{2}(\cos \theta)\right] \\
& \rho_{20}(\theta, \phi)=\sqrt{5} \sigma_{0}\left[s_{2}-2 \alpha_{2} P_{2}(\cos \theta)\right] \\
& \rho_{21}(\theta, \phi)=\sigma_{0} \gamma_{2} \sin \theta \cos \theta e^{i \phi} \\
& \rho_{22}(\theta, \phi)=\sigma_{0} \eta_{2} \sin ^{2} \theta e^{2 i \phi}
\end{aligned}
$$


where $\theta$ and $\phi$ are still defined with respect to the laboratory $Z$-axis. State multipole $\rho_{00}(\theta, \phi)$ is the same in any reference frame, since it describes the isotropic portion of the angular momentum distribution. Multipole $\rho_{20}(\theta, \phi)$ describes the portion of the distribution which is cylindrically symmetric in the recoil frame. It is associated exclusively with incoherent excitation and suggests an angular momentum vector which precesses uniformly around the molecular axis. Since the term containing $s_{2}$ is angle-independent, $s_{2}$ does not contribute to the total laboratory-frame alignment parameter $A_{20}$. Parameter $\alpha_{2}$, on the other hand, is associated with an angular dependence and represents the total contribution to $A_{20}$ from incoherent excitation. ${ }^{4}$

The expressions for $\rho_{20}(\theta, \phi)$ in terms of dynamical functions are proportional to $f_{2}(00)\left[1+2 P_{2}(\cos \theta)\right]$ and $f_{2}(11)\left[1-P_{2}(\cos \theta)\right]$ for parallel and perpendicular transitions, respectively. Since these are proportional to $\rho_{00}(\theta, \phi)$ for the respective limiting values of $\beta_{0}^{\prime}$, the degree of alignment $\left[\propto \rho_{20}(\theta, \phi) / \rho_{00}(\theta, \phi)\right]$ due to incoherent excitation of a single state does not depend on recoil direction. The physical reason for this is clear: Through the interaction $\boldsymbol{\mu} \cdot \mathbf{e}$, only a projection of $\mathbf{e}$ is specified ( scalar), so the molecular-frame vector properties cannot depend on the angle between e and $\boldsymbol{\mu}$ (or $\mathbf{v}$ ). Only the transition probability will depend on this angle. In other words, the overall size but not the shape of the angular momentum distribution can change with recoil angle. Also for incoherent excitation, the expected reflection symmetry in the plane of $e$ and $\boldsymbol{\mu}$ is usually reduced to cylindrical symmetry due to precession of $\mathbf{J}$ about the molecular axis [Fig. 3.1(a)].

In contrast, coherent excitation can produce both a recoil angle dependence and non-cylindrical symmetry of the recoil-frame alignment. These facts are immediately apparent from the latter two expressions in Eqs (3.8): the state multipoles with $q \neq 0$ imply non-cylindrical symmetry, and they clearly have unique angular dependencies. In light of the preceding discussion, we can qualitatively rationalize the angular dependence by viewing a coherent excitation as the interaction of a light vector e with two transition moments. This amounts to the molecule 'knowing' two projections of $\mathbf{e}$ and thus having vector properties which depend on the recoil angle

\footnotetext{
${ }^{4}$ The laboratory-frame expressions [Eqs 3.6 and 3.7] would be used for actual angular integration to give $A_{20}$, but it is not obvious by inspection which terms would disappear.
} 
(a)

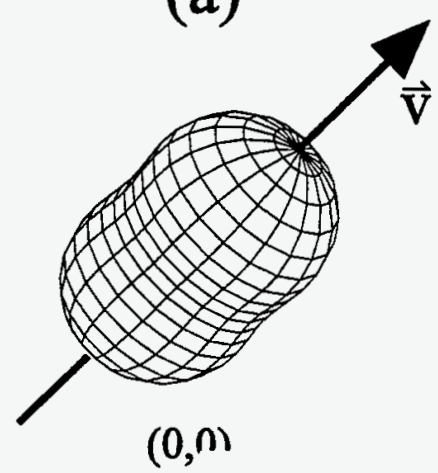

(b)

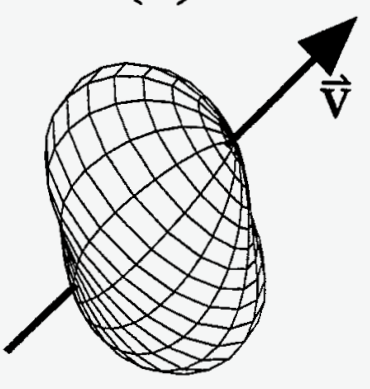

$(1 \mathrm{n})$ (c)

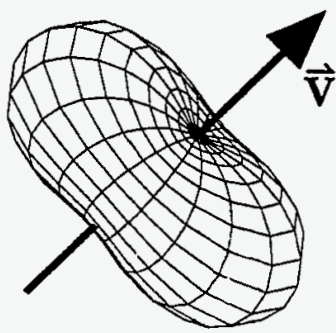

' 1,1$)$

Figure 3.1: Examples of a classical angular momentum distribution $P(\mathbf{J})$ about the recoil vector for $(a)$ incoherent excitation $\left[\left(q, q^{\prime}\right)=(0,0)\right]$, (b) coherent excitation of parallel and perpendicular transitions $\left[\left(q, q^{\prime}\right)=(1,0)\right]$, and $(c)$ coherent excitation of two perpendicular transitions $\left[\left(q, q^{\prime}\right)=(1,-1)\right]$.

$\theta$. A qualitative rationale for the non-cylindrical symmetry comes from viewing the angular momentum vector produced in a coherent excitation as the superposition of the vectors which we would expect for the individual excitations. Even though the individual vectors would precess uniformly, when they are added with a well-defined phase, the resultant may no longer precess uniformly, corresponding to non-cylindrical symmetry. For a $(1,-1)$-type of coherence, both transition moments will still be in the plane perpendicular to the molecular axis, and we would expect the angular momentum distribution to show reflection symmetry through this plane [Fig. 3.1(c)]. Indeed this is true for the $q= \pm 2$ multipoles, which correspond in the classical limit to $d_{x y}$ and $d_{x^{2}-y^{2}}$ spherical harmonic shapes (Cartesian basis). For the (1,0)-type of coherence, the superposition of angular momentum vectors from parallel and perpendicular transitions will lie between $0^{\circ}$ and $90^{\circ}$ to the recoil direction, consistent with a $q= \pm 1$ alignment multipole ( $d_{x z}$ and $d_{y z}$ shapes) with maxima at $45^{\circ}$ to the recoil vector [Fig. 3.1(b)]. 


\subsection{Two-photon Transition Probabilities}

Kummel and coworkers have described a method for extracting orientation and alignment information from measurements which use 2-photon excitation as a probe $[78,79]$. This pair of papers (KSZ1 and KSZ2) provides detailed derivations of the final expressions, but is ultimately intended as a user's guide for experimentalists. We have used their approach with some small modifications. The major results of their work are summarized by the expressions in Table I of KSZ2. We present the main result here in generic form (slightly different from the original) for comparison to our modified version:

$$
\begin{aligned}
I & =C \sum_{k q} P_{q}^{k} \rho_{q}^{k} \\
& =C \sum_{k q}\left\{\sum_{k_{a} k_{d}} \mathcal{S}_{k_{a} k_{d}}^{k}\left[E_{k_{a}} \otimes E_{k_{d}}\right]_{-q}^{k}\right\} \rho_{q}^{k} .
\end{aligned}
$$

The indices $k_{a}$ and $k_{d}$ are the same as in KSZ, and correspond to the tensor ranks for the first and second photons, respectively. ${ }^{5}$ The factor $\mathcal{S}_{k_{a} k_{d}}^{k}$, which is not written as a single factor in $\mathrm{KSZ}$, is a linestrength factor that depends on all the quantum numbers of the initial, intermediate and final states involved in the 2-photon process, but not on the projections of any vectors. All projection information is contained in the state multipoles and the term $\left[E_{k_{a}} \otimes E_{k_{d}}\right]_{-q}^{k}$, which is the combined polarization tensor for both probe photons. The total intensity expression of KSZ2 is the scalar (dot) product of the combined polarization tensor and the state multipole.

The version of the KSZ expression which we use was derived from scratch by Professor Vasyutinskii, but ultimately represents an algebraic rearrangement of the KSZ expression:

$$
I=C \sum_{k k_{a} k_{d}} S_{k k_{a}}^{k_{d}}\left[\left(\rho_{k} \otimes E_{k_{a}}\right)_{k_{d}} \cdot E_{k_{d}}\right] .
$$

This modified version has two advantages-one practical and one conceptual. The practical convenience lies in the complete separation between the scalar linestrength

\footnotetext{
${ }^{5}$ the subscripts $a$ and $d$ presumably originate from $1+1$ laser-induced fluorescence (LIF) notation, where the first photon is absorbed and the second (fluorescence) photon is detected.
} 
factor $S_{k k_{a}}^{k_{d}}$ and the tensor quantities in the photon-atom dot product. In this form, the qualitative dependence of the signal on laser polarization can be studied without reference to linestrengths. Of course, the novelty of this form should not be overemphasized, since in principle, the original KSZ expressions can be rewritten in this way. Nevertheless, this viewpoint is not explicitly considered in KSZ, since the light polarization is absorbed into the factor $P_{q}^{k}$.

The conceptual advantage of Eq. (3.10) relates to the order in which the tensor products are taken. In KSZ, a single tensor product is constructed for both photons, then a dot product is taken between the total polarization tensor and the state tensor of the atomic (or molecular) system. In contrast, the multiplication order in our expression corresponds to the order of photon absorption and provides insight into the way that this absorption modifies the angular momentum polarization of the ensemble. The polarization properties of the first photon are described by the tensor $E_{k_{a} q_{a}}$. The tensor product $(\otimes)$ with the photofragment ensemble described by $\rho_{k q}$ yields an excited state ensemble of rank $k_{d}$. The fact that a full tensor product appears here indicates that the total process is sensitive to the polarization properties of the intermediate ('virtual') state. However, this is not true for the resonant state reached by the second photon, since we assume that the subsequent ionization step is saturated. For this reason, the interaction of the excited intermediate state with the second photon (tensor $E_{k_{d} q_{d}}$ ) is represented by a scalar dot product. In other words, the ionization step probes the population, but not the polarization of the 2-photon-excited state.

At first glance, the combined polarization tensor $\left[E_{k_{a}} \otimes E_{k_{d}}\right]_{-q}^{k}$ from KSZ is appealing because of its apparent symmetry with respect to the first and second photons. But this is misleading for the reasons discussed above, and in fact the true asymmetry between $k_{a}$ and $k_{d}$ is also apparent in the way that these indices appear in the 6-j and 9-j symbols of Eq. (3.12) below. This expression is essentially the same in KSZ. One could argue that the KSZ form is more convenient from the perspective of clean bookkeeping, since all light polarization information is grouped into one term. While this does allow specific instances of the polarization expression to be tabulated 
for common experimental geometries (as in KSZ2), in the end these issues are more a matter of appearance than convenience, since the same terms will result from the dot product no matter which form is used. The tensor product in Eq. (3.10) is easily calculated according to

$$
\left[\left(\rho_{k} \otimes E_{k_{a}}\right)_{k_{d}} \cdot E_{k_{d}}\right]=\sum_{k k_{a} k_{d}}(-1)^{k-k_{a}} \sqrt{2 k_{d}+1}\left(\begin{array}{ccc}
k & k_{a} & k_{d} \\
q & q_{a} & -q_{d}
\end{array}\right) E_{k_{a} q_{a}} \rho_{k q} E_{k_{d}-q_{d}} \text {. }
$$

One need only determine the values for the single photon polarization tensors $E_{k q}$, using the same definition as in Eq. (3.3). These are also tabulated for some often-used probe geometries in Table III of KSZ2.

The linestrength factors $S_{k k_{a}}^{k_{d}}$ in our version are slightly different from those of $\mathrm{KSZ}\left(\mathcal{S}_{k_{a} k_{d}}^{k}\right.$ in Eq. 3.9). This is a consequence of choosing a different convention for the polarization tensor definitions and of carrying out the angular momentum recoupling algebra in a different order. Our linestrength factor is

$$
\begin{aligned}
S_{k k_{a}}^{k_{d}}\left(J_{i} \gamma_{i} ; J_{f} \gamma_{f}\right)= & (-1)^{J_{f}+k+k_{a}+k_{d}+1} \sqrt{(2 k+1)\left(2 k_{a}+1\right)} \\
& \times \sum_{J_{e} \gamma_{e} J_{e}^{\prime} \gamma_{e}^{\prime}}(-1)^{J_{e^{\prime}}}\left\{\begin{array}{ccc}
J_{i} & 1 & J_{e} \\
J_{i} & 1 & J_{e}^{\prime} \\
k & k_{a} & k_{d}
\end{array}\right\}\left\{\begin{array}{ccc}
J_{e} & J_{e}^{\prime} & k_{d} \\
1 & 1 & J_{f}
\end{array}\right\} \\
& \times S\left(J_{i} \gamma_{i}, J_{e} \gamma_{e}, J_{e}^{\prime} \gamma_{e}^{\prime}, J_{f} \gamma_{f}\right) .
\end{aligned}
$$

The indices $i, e$ and $f$ designate initial, excited (intermediate or 'virtual') and final states. The presence of both $e$ and $e^{\prime}$ is a result of coherent sums over different intermediate excited states. The factor $S\left(J_{i} \gamma_{i}, J_{e} \gamma_{e}, J_{e}^{\prime} \gamma_{e}^{\prime}, J_{f} \gamma_{f}\right)$ contains reduced matrix elements (i.e. $M_{J}$-state dependence already extracted) and the familiar energy denominator of second order time-dependent perturbation theory. After further reduction of the matrix elements using Eq. 5.72 of Ref. [73], the factor $S$ of Eq. (3.12) becomes

$$
\begin{array}{r}
S\left(J_{i} \gamma_{i}, J_{e} \gamma_{e}, J_{e}^{\prime} \gamma_{e}^{\prime}, J_{f} \gamma_{f}\right)=\left(2 J_{i}+1\right)\left(2 J_{e}+1\right)\left(2 J_{e}^{\prime}+1\right)\left(2 J_{f}+1\right) \\
\times\left\{\begin{array}{ccc}
L_{e} & S & J_{e} \\
J_{f} & 1 & L_{f}
\end{array}\right\}\left\{\begin{array}{ccc}
L_{e} & S & J_{e} \\
J_{i} & 1 & L_{i}
\end{array}\right\}\left\{\begin{array}{ccc}
L_{e}^{\prime} & S & J_{e}^{\prime} \\
J_{i} & 1 & L_{i}
\end{array}\right\}\left\{\begin{array}{ccc}
L_{e}^{\prime} & S & J_{e}^{\prime} \\
J_{f} & 1 & L_{f}
\end{array}\right\} \\
\times \frac{\left\langle L_{f}\|d\| L_{e}\right\rangle\left\langle L_{f}\|d\| L_{e}^{\prime}\right\rangle^{*}\left\langle L_{e}\|d\| L_{i}\right\rangle\left\langle L_{e}^{\prime}\|d\| L_{i}\right\rangle^{*}}{\left(E_{e i}-h \nu+i \Gamma / 2\right)\left(E_{e^{\prime} i}-h \nu+i \Gamma / 2\right)} .
\end{array}
$$


In order to obtain quantitative linestrength factors, it will be necessary to calculate the reduced matrix elements in Eq. (3.13). Omidvar has reported values of these radial integrals for some states of $\mathrm{Cl}, \mathrm{O}$ and $\mathrm{N}$ atoms, calculated with the 'frozen core' approximation $[67,68]$. However, this calculation has not been done for the specific REMPI transitions used in this work. Also, it is likely that modern computational methods are more accurate. Although the calculation of these integrals is not likely to be terribly challenging (or interesting), in the future we will seek the assistance of atomic structure experts, who are much better equipped than we are to efficiently carry out this task.

At present, there still exist deficiencies in the treatment of transition probabilities represented by Eqs (3.10)-(3.13), which are probably a consequence of implicit approximations in the derivation. There are two problems, which appear to be of a different nature. The first is that the above expressions predict a probability of exactly zero for the two photon transition $\mathrm{Cl}\left(4 \mathrm{p}^{2} \mathrm{~S}_{1 / 2}^{\circ}\right) \leftarrow \leftarrow \mathrm{Cl}\left(3 \mathrm{p}^{2} \mathrm{P}_{3 / 2}^{\circ}\right)$. While the signals that we observed for the ${ }^{2} \mathrm{~S}_{1 / 2}^{\circ}$ line were at least a factor of five smaller than for the ${ }^{2} \mathrm{D}_{3 / 2}^{\circ}$ line, they were clearly not zero. The second failure of the above expressions is that for the ${ }^{2} \mathrm{D}_{3 / 2}^{\circ}$ line, they predict no effect on the signal due to the alignment-a fact which is also contradicted by our measurements. If electron spin is completely eliminated in the derivation, a nonzero alignment contribution for the ${ }^{2} \mathrm{D}_{3 / 2}^{\circ}$ line is obtained, even though this would seem to be a coarser approximation than the forms above. This approximation did not affect the other problem, however-the total calculated signal for the ${ }^{2} \mathrm{~S}_{1 / 2}^{\circ}$ line remained zero. This suggests that the two observed problems in the above treatment have different origins. These problems will obviously need to be solved in order to obtain quantitative values (including the sign) of the alignment anisotropy parameters, however it will become apparent from the discussion of Eq. (3.17) below that a great deal can be learned from knowing their relative size.

We can now write expressions for the $2+1$ REMPI signal for three experimental geometries, corresponding to the probe laser polarization along all three Cartesian 
axes. Using Eqs (3.10) and (3.11) with polarization tensor components

$$
\begin{array}{r}
E_{00}=\frac{1}{\sqrt{3}} ; \quad E_{20}=-\frac{2}{\sqrt{6}} \quad\left(\text { for } \mathrm{e}=e_{Z}\right) \text { and } \\
E_{00}=\frac{1}{\sqrt{3}} ; \quad E_{20}=-\frac{1}{\sqrt{6}} ; \quad E_{2 \pm 2}=-\frac{1}{2} . \quad\left(\text { for } \mathrm{e}=e_{X}, e_{Y}\right)
\end{array}
$$

we get

$$
\begin{aligned}
I_{Z} & =\frac{C}{3}\left[R_{0} \rho_{00}(\theta, \phi)-\sqrt{2} R_{2} \rho_{20}(\theta, \phi)\right] \\
I_{X, Y} & =\frac{C}{3}\left[R_{0} \rho_{00}(\theta, \phi)+\frac{1}{\sqrt{2}}\left\{R_{2} \rho_{20}(\theta, \phi) \mp \sqrt{6} \operatorname{Re}\left[\rho_{22}(\theta, \phi)\right]\right\}\right]
\end{aligned}
$$

where

$$
R_{0}=S_{00}^{0}+2 S_{02}^{2} \quad \text { and } \quad R_{2}=S_{20}^{2}+\frac{1}{\sqrt{5}} S_{22}^{0}+\frac{2}{\sqrt{7}} S_{22}^{2}
$$

The state multipoles here are written as a function of recoil angles, but the expressions are general and $\rho_{k q}$ may be a function of any convenient experimentally-measurable coordinates. The - and + of $\mp$ in Eq. 3.15 correspond to the $X$ and $Y$ probe geometries, respectively. The fact that $\rho_{21}(\theta, \phi)$ does not appear in these expressions is simply a consequence of the experimental geometry. Greatest sensitivity to this state multipole is obtained with the probe laser polarization at $45^{\circ}$ to the $Z$ axis. At $0^{\circ}$ or $90^{\circ}$, there is no sensitivity. Similarly, the imaginary part of $\rho_{22}(\theta, \phi)$ could only be measured with the laser propagating at some angle between the $X$ and $Y$ axes. The fact that these two state multipoles do not contribute to the signal has no relation to whether they exist in the molecular frame. Indeed, any one of the above three expressions contains contributions from all possible molecular-frame multipoles and all possible alignment anisotropy parameters. Measuring more than one geometry is simply an opportunity to reduce the overall uncertainty in the parameters obtained with such an analysis.

Usually, the spatial modulations in an ion image, Doppler profile or TOFMS profile which result from photofragment alignment are a relatively small fraction of the total signal. For this reason, it is useful to isolate the alignment contribution by taking linear combinations of the signal from different geometries, in such a way that the population term $\rho_{00}(\theta, \phi)$ cancels. Furthermore, by combining measurements along 
all three Cartesian axes, the alignment terms can be eliminated. These expressions are

$$
\begin{aligned}
& I_{X, Y}-I_{Z}=\frac{C}{\sqrt{2}} R_{2}\left\{\rho_{20}(\theta, \phi) \mp \sqrt{\frac{2}{3}} \operatorname{Re}\left[\rho_{22}(\theta, \phi)\right]\right\}, \\
& I_{Y}-I_{X}=\frac{2 C}{\sqrt{3}} R_{2} \operatorname{Re}\left[\rho_{22}(\theta, \phi)\right], \\
& I_{X}+I_{Y}+I_{Z}=C R_{0} \rho_{00}(\theta, \phi) .
\end{aligned}
$$

In practice, physical restrictions in the experimental apparatus may prevent measurements at certain geometries. For example, measuring both $I_{X}$ and $I_{Y}$ requires propagating the probe laser beam in two orthogonal directions, which may be impossible in some experimental apparatuses. This problem can be circumvented when the dissociation laser polarization lies parallel to the $Z$-axis (Geometry I), since the $I_{X}$ image is effectively obtained by a simple $90^{\circ}$ rotation of $I_{Y}$. In other words, we can rotate the image instead of the apparatus.

As described in the next section, Eqs (3.17) can be converted from $(\theta, \phi)$ space to two-dimensional image space. It is apparent from Eqs (3.17) that the overall shape of the resulting difference images (or 'alignment images') is independent of the quantitative values of the coefficients $S_{k k_{a}}^{k_{d}}$, since all rank $k$ state multipoles are multiplied by the same linestrength coefficient $R_{k}$. For this reason, a great deal of information can be obtained without knowing quantitative values (or even the sign) of the linestrength coefficients. More specifically, the symmetry of the excited states and whether coherent excitation occurred is apparent from the shapes of the difference images. This corresponds to knowing relative values of the polarization anisotropy parameters. In contrast, determinations of asymptotic state branching rations, which provide information about nonadiabatic transition probabilities and initial excitation probabilities, require quantitative values of the alignment anisotropy parameters.

\subsection{Alignment Image Basis Functions}

The state multipole expressions presented in the previous sections are functions of recoil angles $\theta$ and $\phi$. In general, they are also a function of the recoil speed, 
however at present we consider only $\delta$-function speed distributions. The most common types of experimental data, on the other hand, represent an integration over one or two Cartesian dimensions. Doppler and TOFMS profiles are projections onto the probe laser propagation axis $\left[\iint I(\theta, \phi) d Y d Z\right]$ and detector flight axis $\left[\iint I(\theta, \phi) d X d Y\right]$, respectively. Ion images are projections onto the detector plane $\left[\int I(\theta, \phi) d Z\right]$.

In most ion imaging studies to date, investigators sought to obtain recoil energy and angular distributions, using the inverse-Abel transform to directly invert. their images to obtain the original three-dimensional ion spatial distribution [59]. The forward-Abel transform is actually intended to project a circularly symmetric two-dimensional pattern, $f(r)$ into a one-dimensional function $I(X)$. It has the form

$$
I(X)=2 \int_{X}^{\infty} \frac{f(r) r d r}{\sqrt{r^{2}-X^{2}}}
$$

Since this expression is of one less dimension than what is generally needed for imaging, it can only be used if the image is a projection of a cylindrically symmetric distribution, where the symmetry axis lies horizontally on the two-dimensional surface. Then the inverse Abel-transform can be applied independently to successive pixel rows which are perpendicular to the symmetry axis.

Cylindrically symmetric ion distributions result when linearly or circularly polarized dissociation light and an isotropic gas sample are used, and the detection sensitivity is independent of the probe laser polarization. The latter condition is not met when a $\mathbf{v}$-J correlation exists, so the inverse-Abel transform cannot be used. Instead, we use the expression

$$
I(\rho, \phi)=2 \int_{\rho}^{\infty} \frac{f(\arcsin (\rho / r), \phi) g(r)}{\sqrt{1-\rho^{2} / r^{2}}} d r
$$

where $I(\rho, \phi)$ is the image, $(\rho, \phi)$ are polar coordinates ( $\phi$ is the same as in three dimensions), and $r$ is the length of the photofragment radius vector. These variables are labeled clearly in Fig 3.2. The function $f(\arcsin (\rho / r), \phi) \equiv f(\theta, \phi)$ describes the angular dependence of the intensity distribution and can be substituted with one of the REMPI intensity differences in Eqs (3.17). Eq. (3.19) assumes reflection symmetry of the function $f$ in the $X-Y$ plane. The function $g(r)$ describes the radial dependence 


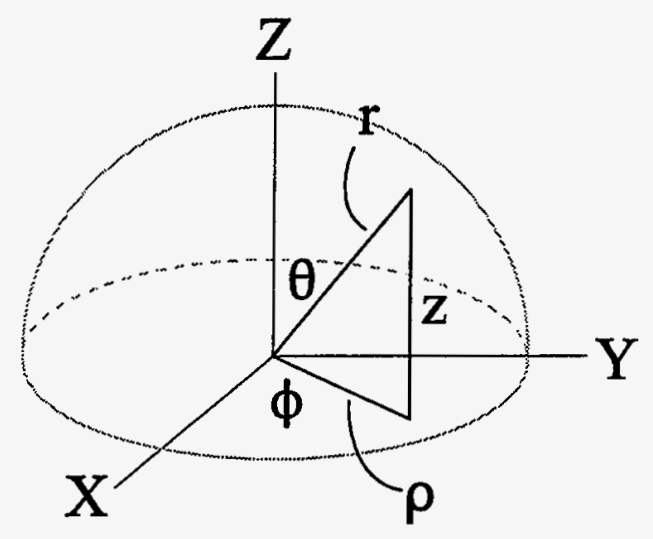

Figure 3.2: Clarification of variables in 3D to 2D transformation (Eq. 3.19).

of the three-dimensional distribution. ${ }^{6}$ Unlike the Abel transform, Eq. (3.19) is not a true integral transform and cannot be inverted. Nevertheless, it may still be used for so-called 'forward-convolution' fitting of image data.

For monoenergetic chlorine atoms, $g(r)=\delta\left(r-r_{0}\right)$, where $r_{0}=v_{0} \tau, v_{0}$ is the photofragment velocity, and $\tau$ is the flight time of the ions. For this special case, we can evaluate the integral in Eq. (3.19) analytically. For dissociation polarization geometries I and II, using the $I_{Z}-I_{Y}$ expression from Eq. (3.17a) for $f$, we get

$$
\begin{aligned}
{ }^{\mathrm{I}} I_{Y Z}(t, \phi) & \propto \frac{2}{\sqrt{1-t^{2}}}\left\{\left[s_{2}-2 \alpha_{2}+3 \alpha_{2} t^{2}\right]\left[1-t^{2}\left(1+\sin ^{2} \phi\right)\right]\right. \\
& -2 \gamma_{2} t^{2}\left(1-t^{2}\right)\left(1+\sin ^{2} \phi\right) \\
& \left.-\frac{\eta_{2}}{4} t^{2}\left[t^{2}+\left(2-t^{2}\right) \cos (2 \phi)\right]\right\}
\end{aligned}
$$

and

$$
\begin{aligned}
{ }^{\text {II }} I_{Y Z}(t, \phi) & \propto \frac{2}{\sqrt{1-t^{2}}}\left\{\left[s_{2}+\alpha_{2}\left(1-3 t^{2} \sin ^{2} \phi\right)\right]\left[1-t^{2}\left(1+\sin ^{2} \phi\right)\right]\right. \\
& +2 \gamma_{2} t^{2} \sin ^{2} \phi\left[2-t^{2}\left(1+\sin ^{2} \phi\right)\right] \\
& \left.+\frac{\eta_{2}}{2}\left[t^{2}\left(2-t^{2}\right) \cos (2 \phi)+1-t^{2}+t^{4}\left(1-\frac{\sin ^{2}(2 \phi)}{4}\right)\right]\right\} .
\end{aligned}
$$

The variable $t$ is the radial coordinate normalized to the maximum possible radius, i.e. $t=\rho / r_{0}$.

\footnotetext{
${ }^{6}$ Most analyses of $\mathbf{v - J}$ correlation data (including this one) assume the separability $h(r, \theta, \phi)=f(\theta, \phi) g(r)$. However in general, vector properties will depend on recoil velocity, so this separation will not be valid.
} 
Eqs (3.20) provide a powerful means for interpreting the alignment contribution to photofragment ion image data, since each of the four alignment mechanisms discussed in Sec. 3.3 is associated with a unique radial and angular dependence in the images. The basis images shown in Fig. 3.3 are the final result of the theoretical approach discussed in this and the preceding sections. Each basis image corresponds to a maximum limiting value for each $k=2$ dynamical function.

The calculated images of Fig. 3.3(a) show the contribution of a pure perpendicular optical transition $\left(\left|\Omega-\Omega_{0}\right|=1\right.$, where $\Omega_{0}$ and $\Omega$ are projections of the total angular momentum onto the internuclear axis for ground and excited states, respectively), while Fig. 3.3(b) corresponds to a pure parallel transition $\left(\Omega-\Omega_{0}=0\right)$. Since the anisotropy parameters are defined according to angular dependence, whereas we are most interested here in mechanistic assignments, Figs 3.3(a) and 3.3(b) correspond to both $s_{2}$ and $\alpha_{2}$ having nonzero values. It is clear from the definitions of the anisotropy parameters that the parallel contribution is obtained with $s_{2}=2 \alpha_{2}$ and the perpendicular contribution from $s_{2}=-\alpha_{2}$. The other two parameters are set to zero. The coherent contributions are shown in the third and fourth rows of the figure. Parameter $\eta_{2}$ corresponds to coherent perpendicular excitation ( $\Omega= \pm 1$ ) [Fig. 3.3(c)], while $\gamma_{2}$ corresponds to coherent excitation via both parallel and perpendicular transitions [Fig. 3.3(d)].

Linear combinations of the shapes in Fig. 3.3 can be used to simulate the alignment contribution to photofragment ion image data from dissociations with linearly polarized light which produce fragments with a single recoil velocity. For chlorine dissociation, which can produce only rank 2 alignment, no other shapes are necessary. In the next chapter, chlorine images are simulated using these basis images. For higher angular momenta, which may be more sharply peaked, additional basis images may be needed, although the rank 2 alignment is likely to be the most important. Real image data will of course also have finite spatial resolution, unlike the calculated images which may become infinite at the edges. (The shapes in Fig. 3.3 were truncated.) Prior to fitting, one could convolute the calculated images with a two-dimensional function which approximates the experimental spatial resolution. Another option would be to use Monte Carlo methods. 


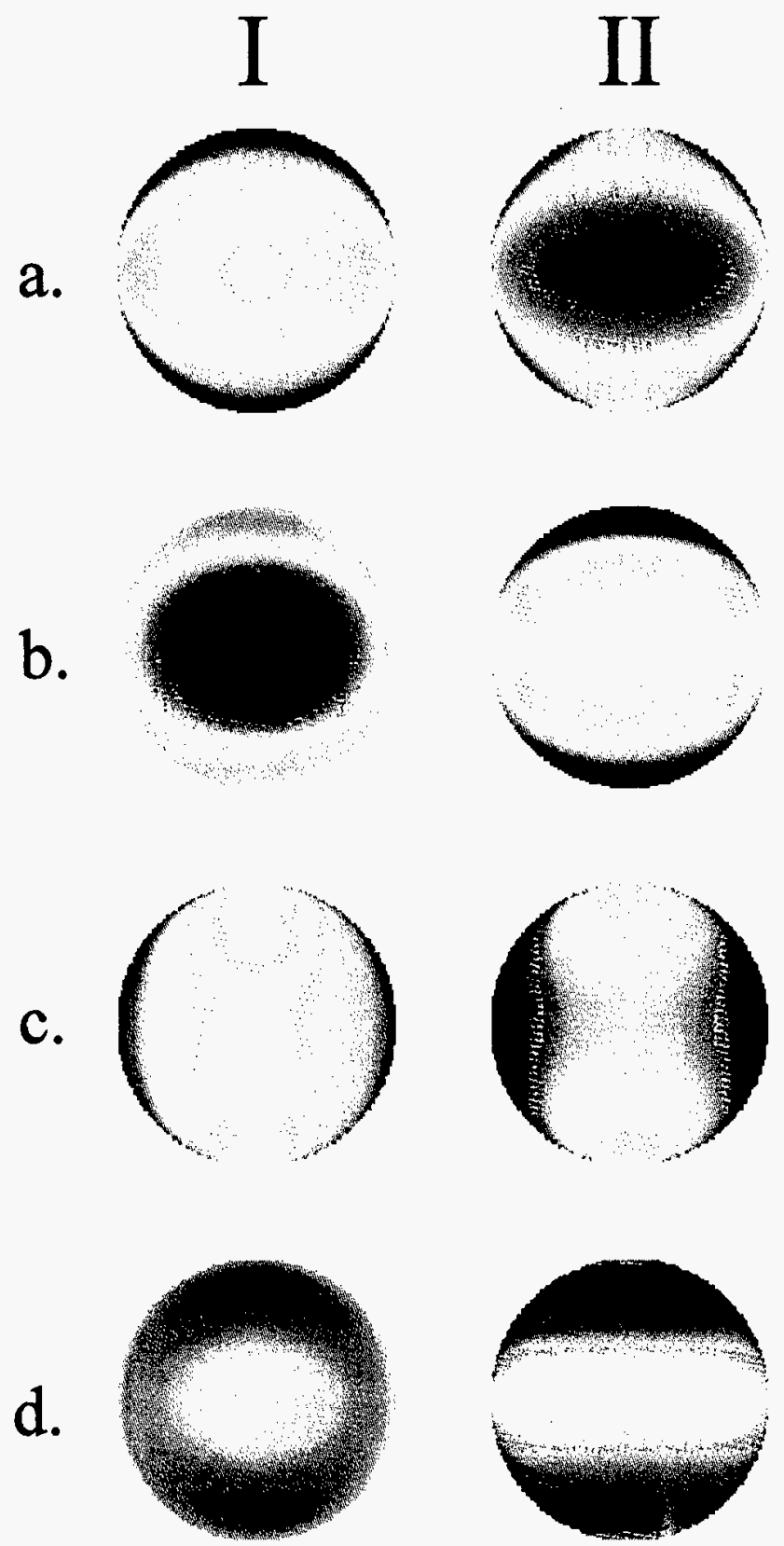

Figure 3.3: (color) Alignment image basis functions: plots of $I_{Y Z}(t, \phi)$ [Eq. (3.20)] for dissociation polarization Geometries I and II. Cases (a)-(d) correspond to the following mechanistic limits: (a) incoherent perpendicular excitation, (b) incoherent parallel excitation, (c) coherent perpendicular excitation, (d) coherent parallel and perpendicular excitation. The corresponding values of the alignment anisotropy parameters are discussed in the text. Blue, red, and white correspond to positive, negative, and zero values, respectively. 


\section{Chapter 4}

\section{Results and Discussion}

\subsection{Overview}

In this chapter, ion imaging results are presented for the dissociation of chlorine at $355 \mathrm{~nm}$ and for ozone at $266 \mathrm{~nm}$. Both systems show clear evidence of angular momentum polarization. The discussion emphasizes the interpretation for chlorine, since a relatively complete theoretical description is possible for diatomic molecules. Although our interpretation for chlorine remains qualitative at present, the existing theory permits, in principle, a quantitative determination of relative excitation probabilities and nonadiabatic transition probabilities. Even the qualitative results presented in this chapter point clearly to a mechanism which involves incoherent and coherent perpendicular excitation of the parent molecule and nonadiabatic transitions in the asymptotic region of the potential energy curves. The ozone results presented in this chapter are particularly intriguing, since they exhibit resolved vibrational levels of the oxygen molecular partner. A preliminary analysis indicates that the angular momentum polarization depends on the partner vibrational level. The theoretical tools for interpreting atomic photofragment polarization in polyatomic systems are in the early stages of development, and it appears that ozone dissociation at $266 \mathrm{~nm}$ will be a very promising system with which to test these new theories. 


\subsection{Introduction \& Background: Chlorine Pho- todissociation}

As discussed at the outset, the primary rationale for studying atomic photofragment polarization at this stage is to gain a deeper understanding of the physical process in general. Therefore, we choose simple model systems with a minimum of variables which influence the polarization. We hope that in the future this property can be used as a tool for better understanding the electronic structure and dissociation dynamics of molecules which are important in specific technological or environmental processes. Of course, we should consider that the model systems themselves may have some practical relevance, and indeed this is true for the chlorine molecule.

Free chlorine atoms are known to play a role in the destruction of stratospheric ozone. Some chlorofluorocarbons and chlorohydrocarbons, which were widely used in the past as solvents, for making aerosols, and as refrigerants, are known to be the primary anthropogenic source of chlorine atoms, through photolysis by ultraviolet sunlight. In the past decade, worldwide production and use of these compounds has been curbed as nations have become aware of their danger, and many investigators have turned their attention to other atmospheric processes, such as chemical reactions on the surface of ice clusters in polar stratospheric clouds. One such process is the surface-catalyzed production of chlorine molecules through the reaction of adsorbed hydrochloric acid with gas phase chlorine nitrate. The resulting gas phase chlorine may then photolyze in sunlight. From this perspective, the low-lying electronic states and dissociation dynamics of this molecule are of very practical interest. On a more detailed level, it is known that the polarization of an atom affects its reactivity with other molecules (see e.g. Refs [80,81]), although it is less clear that a substantial effect would remain after detailed cross sections are averaged to obtain thermal rate constants.

The dominant feature of the ultraviolet absorption spectrum of chlorine [Fig. 4.1] is a wide continuum with its maximum around $330 \mathrm{~nm}$. This feature results from excitation to the dissociative $\widetilde{C}^{1} \Pi_{1 u}$ state of chlorine. A nominally spin- 


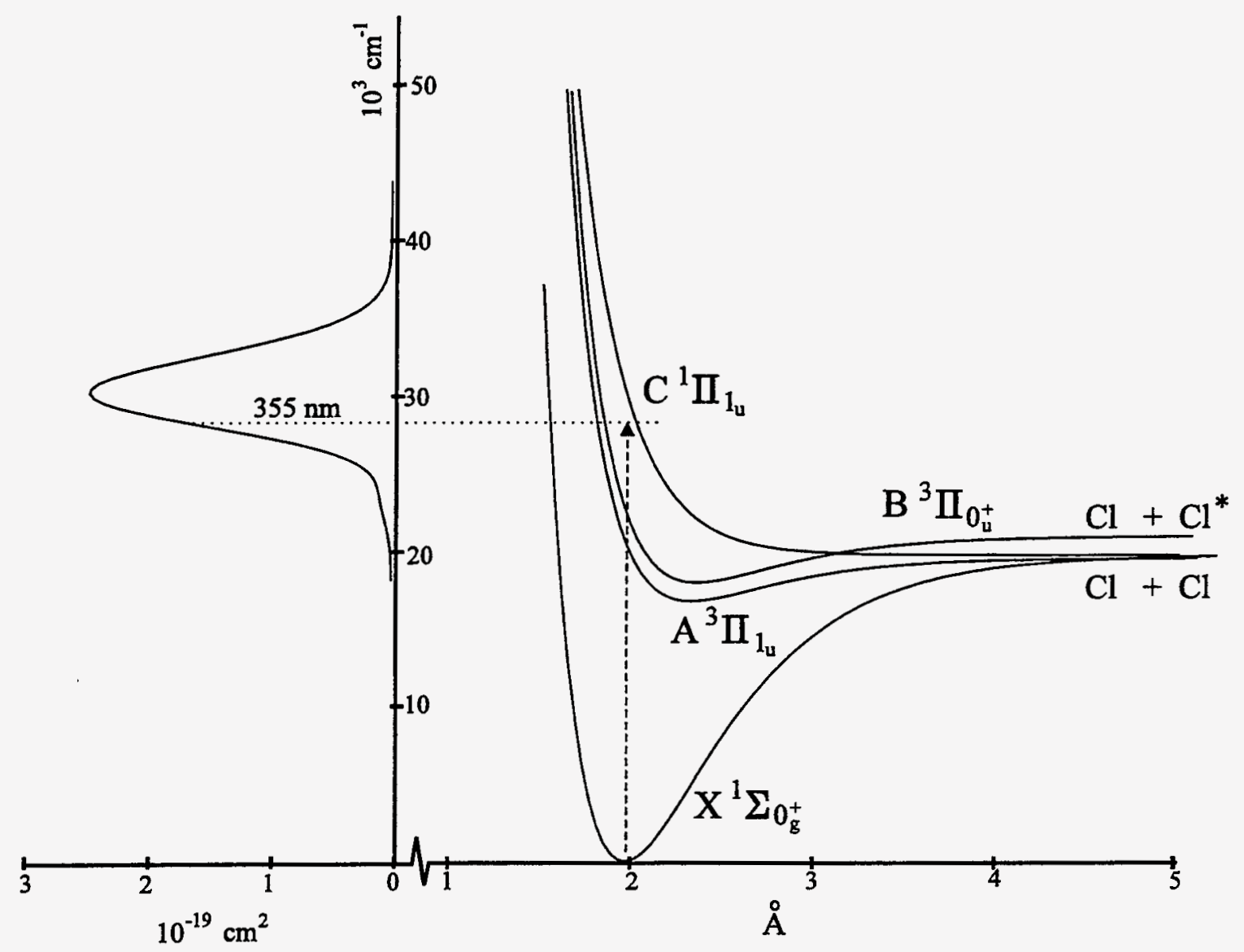

Figure 4.1: Absorption spectrum and potential energy curves (qualitative) for the chlorine molecule. 
forbidden transition at longer wavelengths results in a weak shoulder with resolved structure above $476 \mathrm{~nm}$. This feature is assigned to the state $\tilde{B}^{3} \Pi_{0_{u}^{+}}$. Recently, Maric et al. [82] have published quantitative measurements of the chlorine UV absorption spectrum at room temperature and fit the measured spectrum with a simple semiempirical form. Their spectral resolution was $0.2 \mathrm{~nm}$ for the continuum region and $0.04 \mathrm{~nm}$ for the structured region above $476 \mathrm{~nm}$. They discuss the quality of their measurement and discrepancies between theirs and the original measurements of the 1920 s and ' 30 s. $^{1}$

The lowest few excited electronic states of the chlorine molecule, which are of interest for this study, result from single-electron promotion from a $\pi_{g}$ antibonding orbital to a $\sigma_{u}^{+}$antibonding orbital (see Fig. 1.4). A state correlation diagram based on the general rules of Mulliken [83, 84] is shown in Fig. 4.2. (This diagram also appears explicitly in Refs $[85,86]$.) The lines connecting the molecular and atomic states are adiabatic correlations, corresponding to the electronic energy levels for fixed nuclei (i.e. in the Born-Oppenheimer approximation). The state $\widetilde{C}^{1} \Pi_{1 u}$ correlates adiabatically to two ground state $\left({ }^{2} P_{3 / 2}^{o}\right)$ chlorine atoms $\left(D_{0}=57.1 \mathrm{kcal} / \mathrm{mol}\right)$, while the nearby $\widetilde{B}^{3} \Pi_{0_{u}^{+}}$state, which is responsible for the weak red shoulder in the absorption spectrum, correlates to one ground state and one spin-orbit excited state $\left({ }^{2} P_{1 / 2}^{o}\right)$ atom. (The spin-orbit splitting for chlorine atoms is $881 \mathrm{~cm}^{-1}$.) Potential energy curves for the ground electronic state and the first three excited states are shown in Fig. 4.1.

Chlorine dissociation has been the subject of investigations since the younger days of the chemical reaction dynamics field. The original reports of translational spectroscopy measurements by Busch et al. [10] and Diesen et al. [11] were both for the chlorine molecule. ${ }^{2}$ By monitoring signal both parallel and perpendicular to the laser polarization, both studies showed that a perpendicular transition dominates the absorption. Although both apparatuses were only roughly calibrated, Busch et al. were able to correctly identify the ground state atomic products from their kinetic

\footnotetext{
${ }^{1}$ Ref. 23 of Maric et al. [82] provides a quaint example of getting by on a shoestring before the days of big science: a car headlight lamp was used as a light source.

${ }^{2}$ The competitive spirit in scientific research is evident in the footnotes of both of these papers.
} 


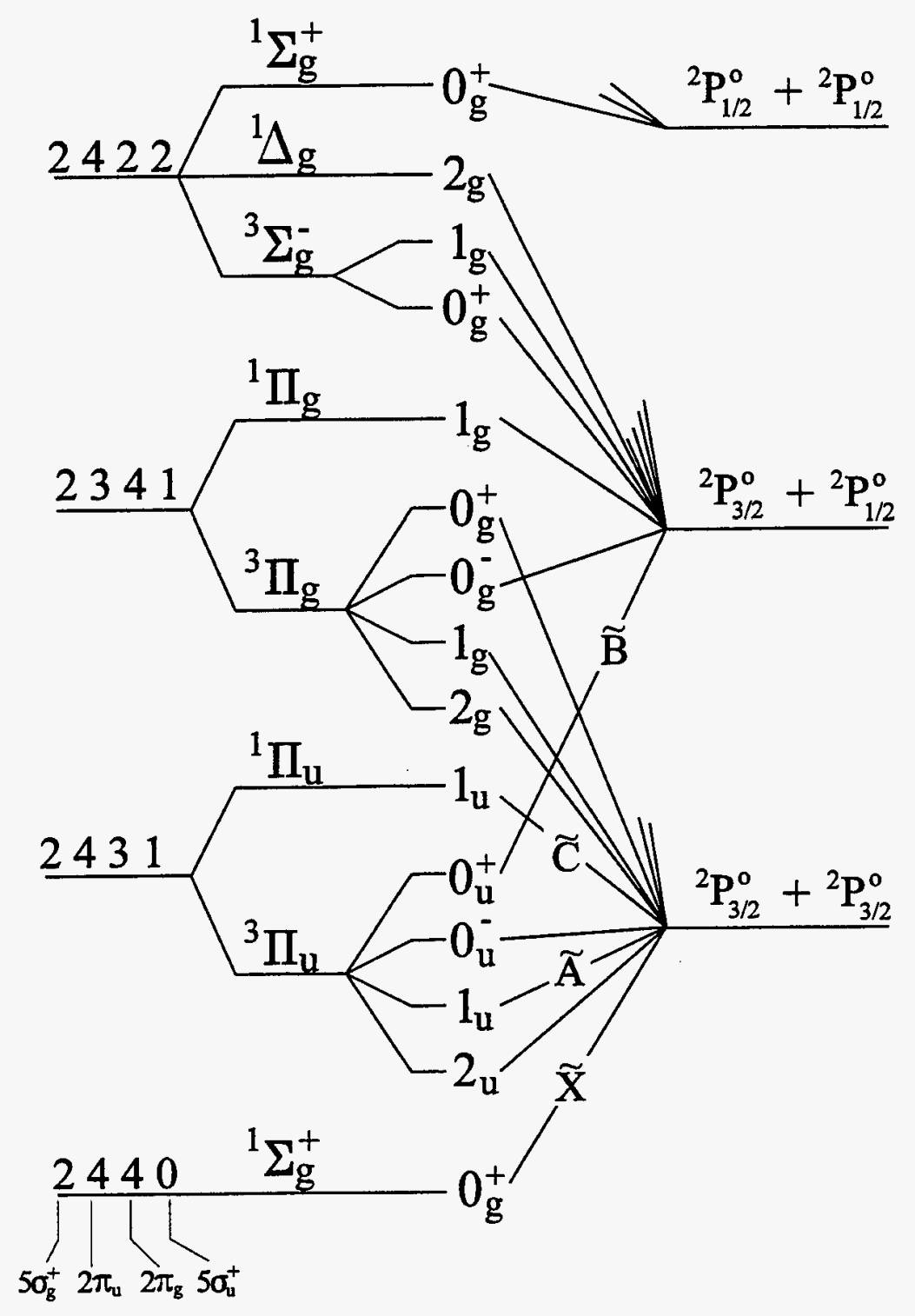

Figure 4.2: State correlation diagram for the chlorine molecule. Molecular orbital configurations are at the far left. Hund's case $a$ and $c$ molecular states are in the next two columns, and atomic states are at the far right. 
energy release.

Almost twenty years later, the ultraviolet dissociation of chlorine began to receive renewed attention - probably this time because of the availability of quantum state-selective detection methods. Li et al. [86] measured a 3+1 REMPI spectrum of chlorine atoms from $323-331 \mathrm{~nm}$. Based on the absence of signal from $\mathrm{Cl}^{*}\left({ }^{2} P_{1 / 2}^{2}\right)$ lines known to exist in this region, they concluded, in agreement with the earlier studies, that the dissociation occurred adiabatically along the $\widetilde{C}^{1} \Pi_{1 u}$ state. Matsumi et al. $[85,87]$ used $2+1$ REMPI to measure Doppler profiles for an extensive study of chlorine dissociation at 19 wavelengths between $266 \mathrm{~nm}$ and $485 \mathrm{~nm}$. For the first time, they directly observed signal from $\mathrm{Cl}^{*}\left({ }^{2} P_{1 / 2}^{2}\right)$. They were able to extract $\beta$ anisotropy parameters, and with careful calibration, the spin-orbit branching ratios $\left[\mathrm{Cl}^{*}\right] /[\mathrm{Cl}]$, over the range of dissociation wavelengths. At $355 \mathrm{~nm}$ the ground state $\mathrm{Cl}$ atoms showed a limiting value of $\beta=-1$ (from adiabatic $\widetilde{C}$-state dissociation), while the $\mathrm{Cl}^{*}$ atoms had a nonlimiting value of $\beta=1.8$ (mostly adiabatic $\widetilde{B}$-state dissociation with a small portion via a nonadiabatic transition from the $\widetilde{C}$-state).

Two recent measurements have contradicted the conclusion that a parallel transition makes a negligible contribution to overall absorption and to ground state $\mathrm{Cl}$ production. Samartzis et al. recently reported non-limiting values for $\beta$ obtained from an ion imaging experiment [88]. These results were confirmed by Rakitzis et al., who used the core extraction time-of-flight method [57]. The former also report a spin-orbit branching ratio five times larger than those of Ref. [85]. These observations suggest stronger absorption by the $\widetilde{B}$-state than was first believed.

Eventually, quantitative measurements of atomic polarization, particularly $\mathrm{Cl}^{*}$ orientation, may help to resolve the above-mentioned disagreements on the $\widetilde{B}$ - and $\widetilde{C}$-state roles in $\mathrm{Cl}$ and $\mathrm{Cl}^{*}$ production, respectively. In studying atomic polarization at this stage, however, we are more concerned with the dominant process-namely, $\mathrm{Cl}$ produced by the $\widetilde{C}$-state. The most significant report in this area is that of Wang et $a l$., who were the first to report the $\mathbf{v - J}$ correlation in chlorine dissociation [55]. They measured TOFMS profiles of $\mathrm{Cl}$ photofragments detected with $2+1$ REMPI. As in our own study, the difference between data collected with different probe polarizations provided evidence of a $\mathbf{v}-\mathbf{J}$ correlation. They correctly interpreted their results as a 
preference for $\left|M_{J}\right|= \pm 1 / 2$ in the recoil frame, based on a simplified fitting procedure involving 'effective' $\beta$ parameters. However, despite a detailed theoretical exposition based on the work of Band et al. [89, 90], they provided no rigorous connection to the measured polarization effect and no discussion of coherence effects. Thus when we received a preprint of their report in 1995, we nevertheless decided to proceed with our own study of $\mathrm{Cl}$ photofragment polarization.

Chlorine dissociation has recently received attention from Rakitzis, Zare and coworkers, who have studied bimolecular reactions between chlorine atoms and hydrocarbons, where the atoms are produced by molecular chlorine photolysis (see e.g. Ref. [91]). A natural question in such studies is whether the atomic polarization affects the bimolecular reaction cross section, and in addressing this question, Rakitzis and Zare have begun a more detailed study of the chlorine dissociation itself [57]. Their current REMPI-TOFMS studies of chlorine dissociation at $320 \mathrm{~nm}$ show both cylindrically symmetric $\left(A_{20}\right)$ and asymmetric $\left(A_{22}\right)$ alignment components in the recoil frame, consistent with our observation (discussed below) of incoherent and coherent alignment mechanisms.

Even though it was first studied almost thirty years ago, the chlorine molecule continues to hold the attention of modern researchers. Because of its simplicity, it has served as a model system for many types of studies, including the development of photofragment translational spectroscopy, early applications of REMPI-based Doppler and TOFMS methods and most recently for the study of orbital alignment. From an experimental perspective, it is a relatively straightforward molecule to study and often serves as a 'practice' system for those testing a new apparatus or new technique-indeed this was true in our laboratory as well. However, the flip side of studying simple systems is that one quickly sees through the usual predictable characteristics to face their more complex and subtle features. Ongoing interest in the hydrogen atom and hydrogen molecule is the strongest evidence of this fact. 


\subsection{Experimental Results on Chlorine Dissocia- tion at $355 \mathrm{~nm}$}

\subsubsection{Chlorine Ion Image Data}

As discussed in Sec. 2.4, it is useful in studies of $\mathbf{v - \mathbf { J }}$ correlations to measure images for four different experimental geometries. These correspond to orienting the dissociation and probe laser polarizations each parallel and perpendicular to the $Z$ axis, which is defined by the ion flight axis. As before, the $Z$ and $Y$ polarizations of the dissociation laser are identified as Geometries I and II, respectively. For all measurements, the dissociation and probe lasers were counterpropagating along the $X$ axis. Ion image data for chlorine dissociation in these four geometries are shown in Figs 4.3 and 4.4. These data have been 'symmetrized' as described in Sec. 2.3.3.

At the bottom of Figs 4.3 and 4.4 are the difference images, or 'alignment images', which represent the alignment contribution to the signal (see Sections 3.4 and 3.5). These were obtained from a properly weighted subtraction of data images collected in the $Z$ and $Y$ probe geometries. Sec. 4.3 .2 discusses normalization of the data images for subtraction, through measurements of total laboratory-frame alignment $A_{20}$. Sec. 4.3.3 discusses qualitative simulations of the alignment images and the quantitative quality of the data images.

\subsubsection{Total Alignment Measurement}

Calculating the difference between the data images $I_{Z}(\rho, \phi)$ and $I_{Y}(\rho, \phi)$ to obtain an alignment image is not trivial, since their relative integrated intensities are not immediately known. In an ideal experiment, where no intensity-altering conditions change between the $Z$ and $Y$ measurements, it would be possible to directly subtract the measured images. In practice this is nearly impossible, unless one carries out a shot-to-shot rotation of the probe laser polarization (without laser beam walk) and collects two separate images in parallel, which would average out slow drifts in experimental conditions. Such a measurement requires a Pockels cell or photoelastic 
modulator and a special frame grabber. While the latter may exist commercially, it was not available to us for the experiments considered here.

As an alternative to simultaneous direct subtraction of the images, we measured total signal intensities (not images) for each relative laser polarization and used these intensities to normalize images which had been collected previously. We used a $355 \mathrm{~nm}$ half-wave plate to rotate the polarization of the dissociation laser, while keeping the probe laser fixed. Data collection consisted of toggling the half-wave plate position every 10-20 seconds, with the laser blocked for 5-10 seconds during changes in order to measure the baseline. Signals were recorded by placing a photomultiplier behind the phosphor screen of the imaging detector and using a boxcar integrator to select the chlorine peak from the resulting mass spectrum. A typical signal trace is shown in Fig. 4.5.

Because of drifts in experimental conditions, including the pulsed molecular beam and laser intensities, we averaged around 100 such 'toggles' to improve the measurement statistics. Unfortunately, some of the experimental drifts were on the timescale of our toggle frequency, while some were even slower, so the final standard deviations are still rather large. Nevertheless, we did not stretch our patience to improve this measurement, since it was sufficient for the current qualitative analysis. In the future, a shot-to-shot polarization rotation with a Pockels cell should rapidly average out the slow drifts as well as improve the shot statistics.

From these measurements, we have determined the following ratios of intensities for parallel and perpendicular relative orientations of the dissociation and probe laser polarizations:

$$
\begin{array}{lll}
{ }^{2} D_{3 / 2}^{o} & \text { state: } & I_{\|} / I_{\perp}=0.91 \pm 0.03 \\
{ }^{2} S_{1 / 2}^{o} & \text { state: } & I_{\|} / I_{\perp}=1.04 \pm 0.04
\end{array}
$$

In weighting the images for subtraction, it should be noted that $I_{Z}$ corresponds to $I_{\|}$ in Geometry I but to $I_{\perp}$ in Geometry II.

A total alignment measurement of the general type described above is what would conventionally be used to obtain values of the alignment parameter $A_{20}$ for the 


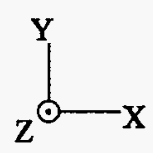

I
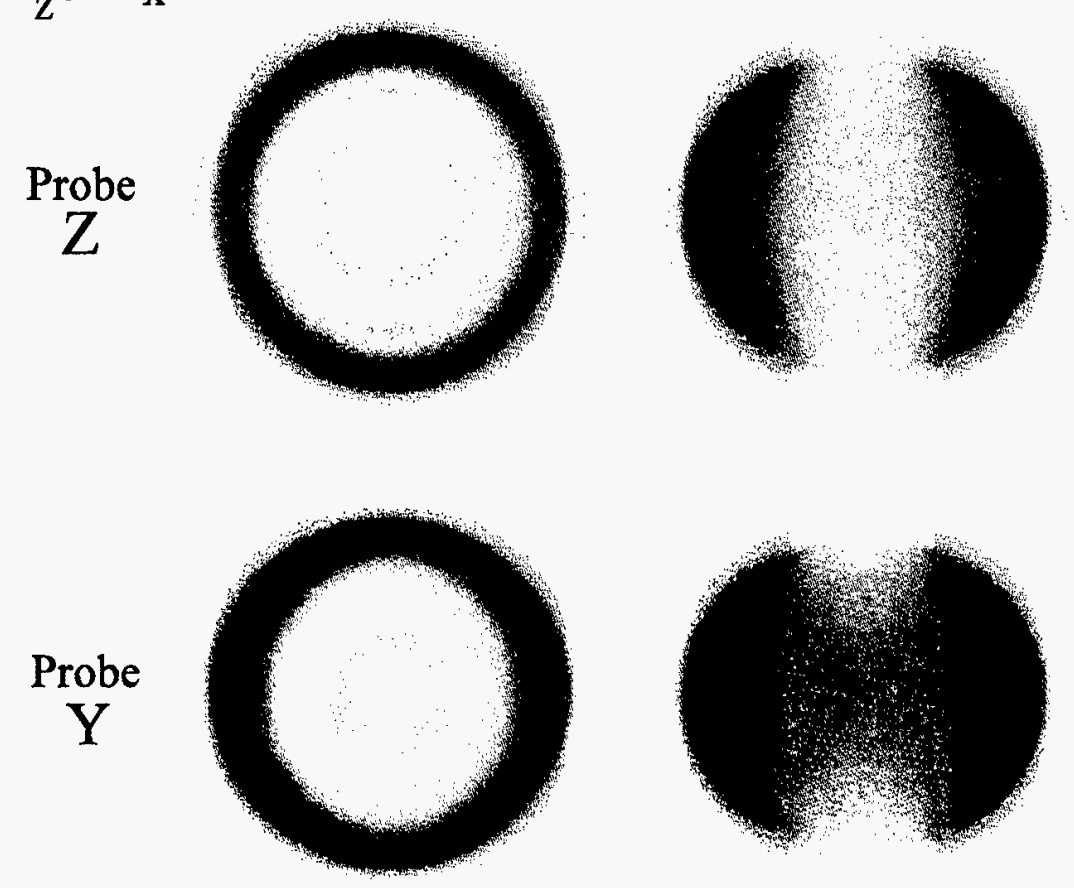

Z-Y

II
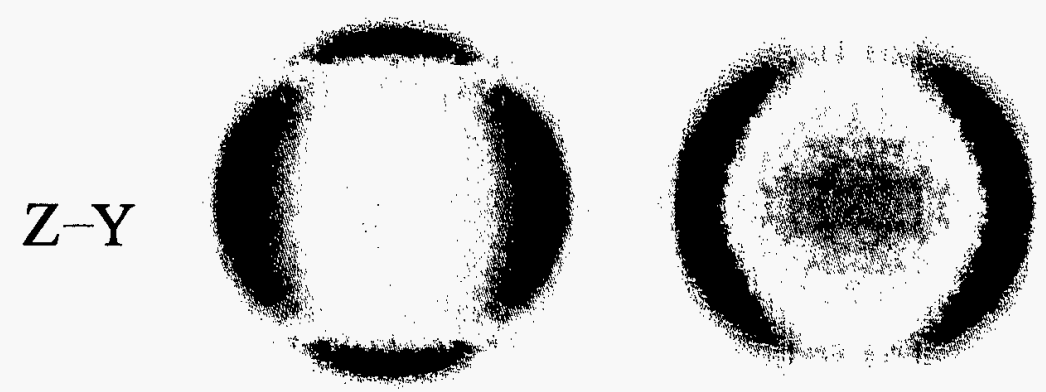

Figure 4.3: (color) Photofragment ion images of $\mathrm{Cl}\left({ }^{2} P_{3 / 2}^{o}\right)$ atoms from the dissociation of chlorine molecules $\left({ }^{2} D_{3 / 2}^{\circ}\right.$ probe state). Geometries I and II correspond to the dissociation laser parallel and perpendicular to the detector flight axis $(Z)$, respectively. The probe laser was polarized along either the $Y$ or $Z$ axis. Colored difference images are proportional to the signal contribution from photofragment alignment. Blue, red, and white correspond to positive, negative, and zero values respectively. 


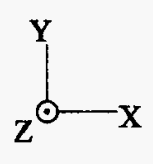

I
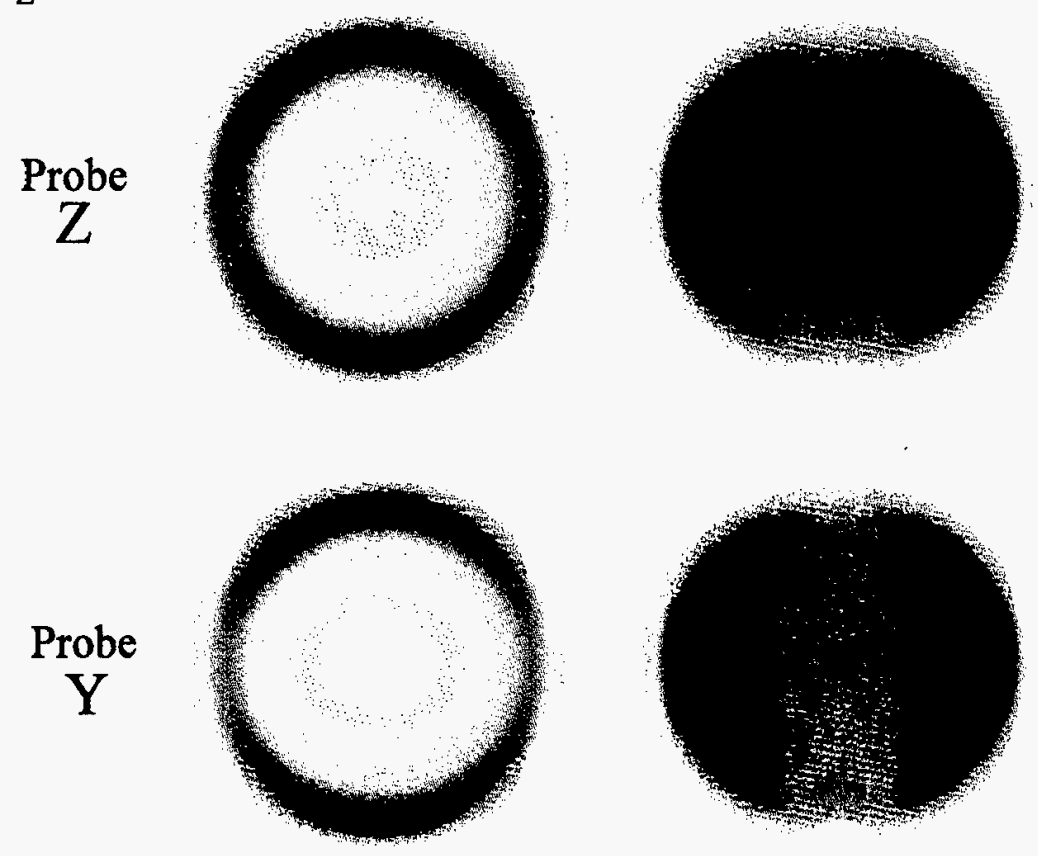

II

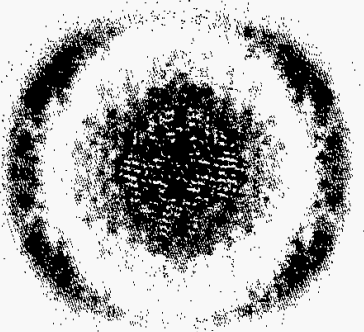

Figure 4.4: (color) Photofragment ion images from the dissociation of chlorine molecules. $\mathrm{Cl}\left({ }^{2} P_{3 / 2}^{\circ}\right)$ atoms were probed with $2+1 \mathrm{REMPI}$ via ${ }^{2} S_{1 / 2}^{\circ}$ state at $235 \mathrm{~nm}$. See Fig. 4.3 caption for further information. 


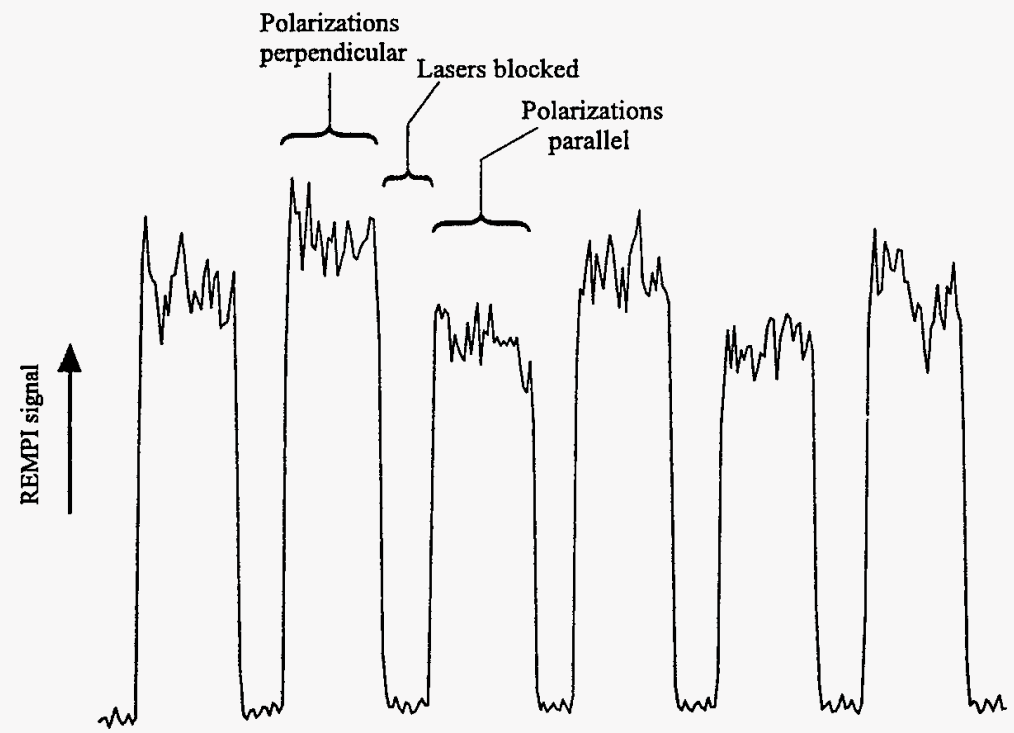

Figure 4.5: Total alignment measurement. Shown is a segment of raw data for measurement of the relative intensities when dissociation and probe laser polarizations are parallel and perpendicular. This ratio is used for normalization of images $I_{Z}(\rho, \phi)$ and $I_{Y}(\rho, \phi)$ prior to subtraction. See text for details.

total photofragment ensemble. The well-known relationship

$$
A_{20} \propto \frac{\left\langle I_{Z}\right\rangle-\left\langle I_{Y}\right\rangle}{\left\langle I_{Z}\right\rangle+2\left\langle I_{Y}\right\rangle}
$$

results from the ratio of Eqs (3.17a) and (3.17c) when the multipole moments are integrated over angles for a cylindrically symmetric distribution. The values of the above ratio from our calibration were $0.032 \pm 0.012$ and $-0.013 \pm 0.013$ using the $\mathrm{D}$ and $\mathrm{S}$ states, respectively. Of course, to calculate the alignment parameter one also needs the proportionality constant, which depends on the values of the linestrength factors $R_{2}$ and $R_{0}$. The total alignment parameter for atomic photofragments provides information on the dissociation mechanism, as shown by Kuprianov and Vasyutinskii [45]. However, $A_{20}$ provides only the sum of alignment anisotropy parameters $\alpha_{2}+\gamma_{2}+\eta_{2}$, rather than the value of each parameter independently. In this sense, it is not possible to explicitly isolate the effect of coherent excitation in a total alignment measurement as one can when the angular distribution of the alignment is measured.

One aspect of angular momentum polarization which has not been mentioned so far is the role of nuclear spin in reducing the polarization of an ensemble 


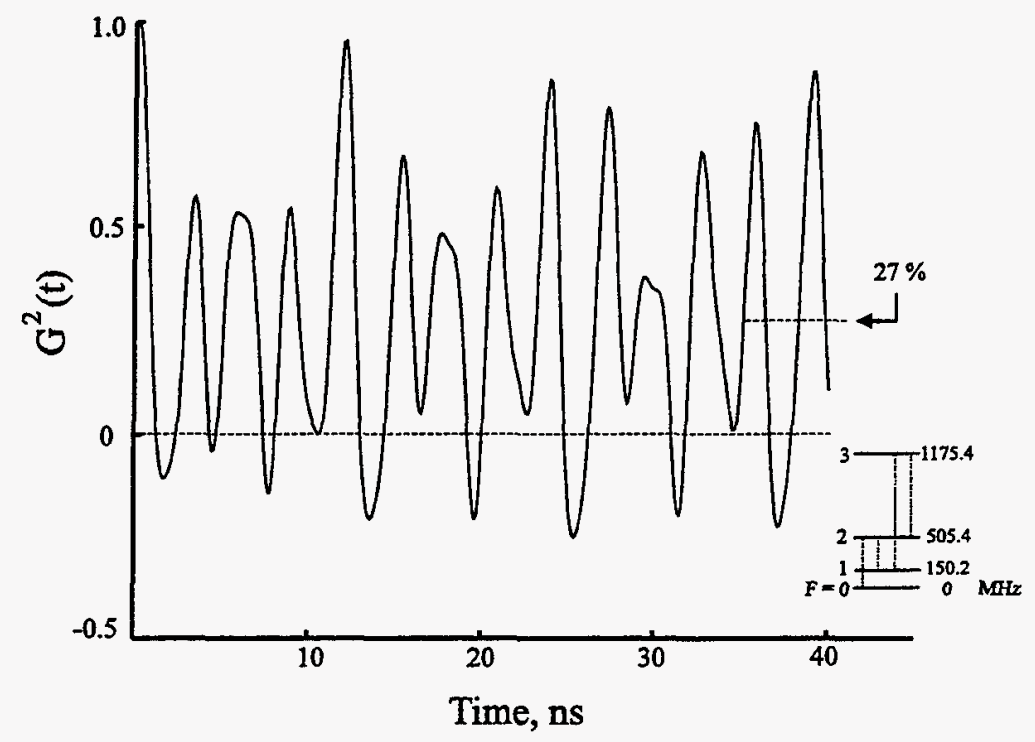

Figure 4.6: Simulated time-dependence of alignment due to interaction with nuclear spin $(I=3 / 2) . G^{2}(t)$ is the factor by which rank 2 alignment changes with time. For the relatively long ( $8 \mathrm{~ns}$ ) excitation source used in these experiments, the oscillations average to a constant depolarization of $27 \%$.

(see e.g. [73]). Nuclear spin $\mathbf{I}$ is weakly coupled to the electronic angular momentum J. According to the vector model of angular momentum coupling, this corresponds to a slow precession of $\mathbf{J}$ around the total angular momentum vector $\mathbf{F}=\mathbf{I}+\mathbf{J}$. This has the practical consequence that a polarized ensemble of $\mathbf{J}$ 's becomes 'washed out'.

The precession times are determined by the splittings between the hyperfine levels. If the laser pulse used to initiate the polarization is short compared to the precession times, then the polarization of $J$ will oscillate according to

$$
G^{k}(t)=\sum_{F, F^{\prime}} \frac{\left(2 F^{\prime}+1\right)(2 F+1)}{2 I+1}\left\{\begin{array}{ccc}
F^{\prime} & F & k \\
J & J & I
\end{array}\right\}^{2} \cos \left[2 \pi\left(E_{F^{\prime}}-E_{F}\right) t / h\right]
$$

This behavior is shown in Fig. 4.6 for ground state ${ }^{35} \mathrm{Cl}$. This atom has hyperfine levels $F=0-3$. Four frequencies contribute to the oscillation-the fastest with a 0.85 ns period and the slowest with 2.8 ns. If the excitation time is long, as it is for our $8 \mathrm{~ns} \mathrm{Nd:YAG} \mathrm{pulses,} \mathrm{then} \mathrm{the} \mathrm{different} \mathrm{frequencies} \mathrm{rapidly} \mathrm{dephase} \mathrm{and} \mathrm{a} \mathrm{constant}$ depolarization is reached. This value equals the sum of the constant terms of Eq. 4.2, which is 0.27 for ground state chlorine. The practical implication of this for our ex- 
periments is that the measured polarization, either as total alignment or as images, will be only about one fourth as strong as the nascent polarization. Since the depolarization process itself is not of interest in most reaction dynamics studies, this correction factor is usually absorbed into the REMPI linestrength factors.

\subsubsection{Alignment Images}

The difference images shown at the bottom of Figs 4.3 and 4.4 were obtained by an appropriately weighted subtraction of the data images with $Z$ and $Y$ probe polarizations. The qualitative appearance of these differences are roughly the same for the ${ }^{2} D_{3 / 2}^{o}$ and ${ }^{2} S_{1 / 2}^{o}$ state measurements. This must be true, since the choice of REMPI transition affects only the relative sensitivity to rank $k=2$ alignment, but not sensitivity to the specific components (specified by $q$ ) of a given rank. Thus we see a sign change between ${ }^{2} D_{3 / 2}^{\circ}$ and ${ }^{2} S_{1 / 2}^{\circ}$, but the general shape remains the same. Likewise, the integrated intensity of these difference images $\left(\propto A_{20}\right)$ was shown earlier to be different.

In order to get a better idea of the data quality, it is preferable to view $X Y Z$ contour plots of the difference images. These plots are shown in Figs 4.7 and 4.8, with the ${ }^{2} D_{3 / 2}^{o}$-state plot inverted in order to make it easier to compare with the ${ }^{2} S_{1 / 2}^{o}{ }^{-}$ state plot. It is clear that the data from ${ }^{2} D_{3 / 2}^{o}$ and ${ }^{2} S_{1 / 2}^{o}$ state measurements, while similar in shape, are not identical. There are two likely reasons for this. The first is the substantial uncertainty in the total alignment calibration discussed earlier. While those uncertainties were only a few percent of the total image intensities (since the total polarization is relatively small), this translates into a substantial uncertainty in the resulting difference images. A comparable source of uncertainty results from imperfections in the detector and is emphasized by the standard deviations on the right side of Figs 4.7 and 4.8. These standard deviations are associated with the fourfold symmetrization discussed in Sec. 2.3.3. Although the experimental uncertainties are substantial, they are one half or less of the measured difference signal. This is more than sufficient for the qualitative analysis discussed in this section and in Sec. 4.4.

As discussed in Sec. 3.5, an observed alignment image can be simulated as a 


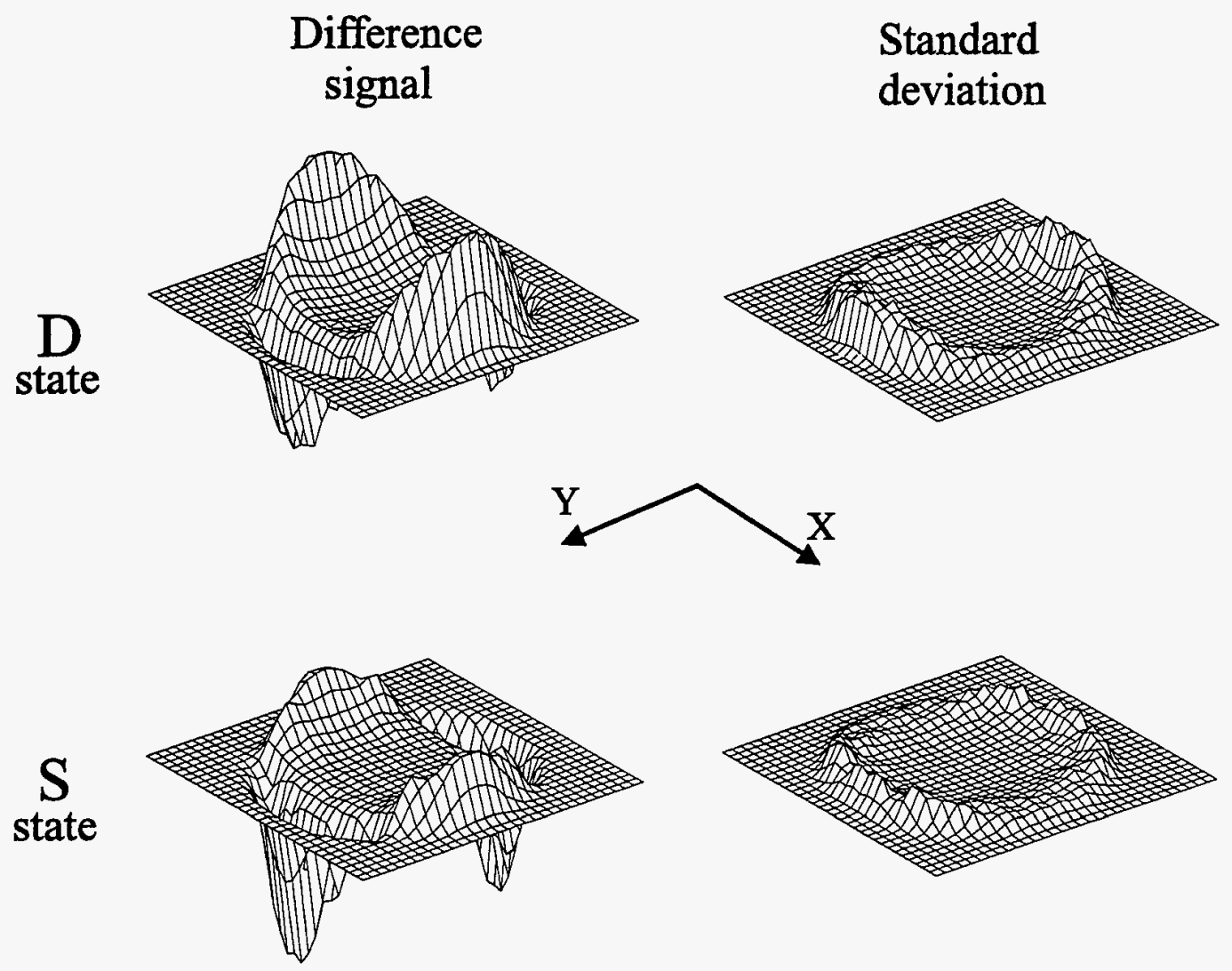

Figure 4.7: Contour plots for Geometry I, emphasizing standard deviation, ${ }^{2} D_{3 / 2}^{\circ}$ and ${ }^{2} S_{1 / 2}^{\circ}$ REMPI states. Difference signals (alignment images) are the same as those shown in the left columns of Figs 4.3 and 4.4. Standard deviations correspond to the averaging of four quadrants by 'symmetrization' of the raw data. ${ }^{2} D_{3 / 2}^{o}$-state signal is inverted for comparison to ${ }^{2} S_{1 / 2}^{o}$-state signal. 


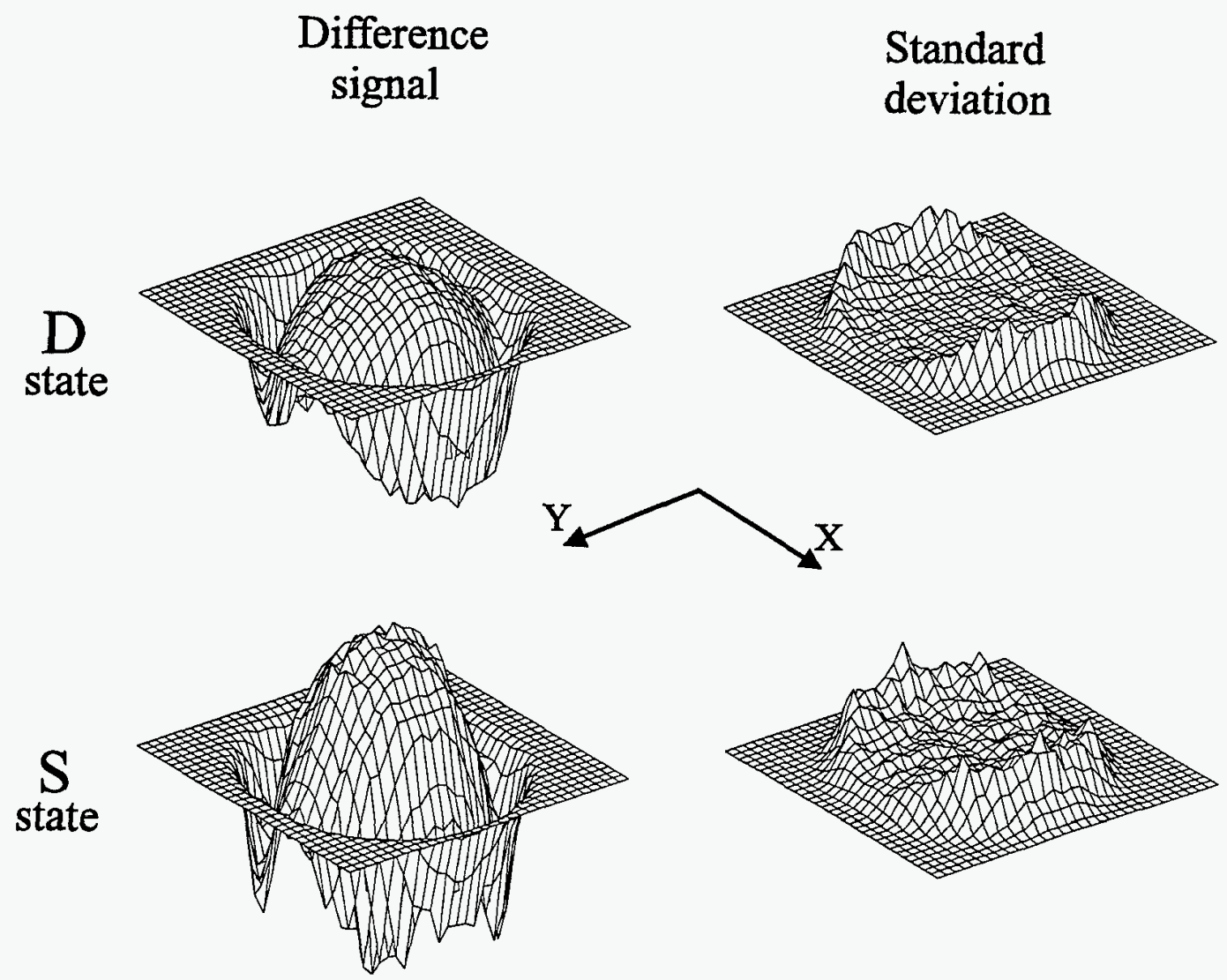

Figure 4.8: Contour plots for Geometry II emphasizing standard deviation, ${ }^{2} D_{3 / 2}^{o}$ and ${ }^{2} S_{1 / 2}^{\circ}$ REMPI states. Difference signals (alignment images) are the same as those shown in the right columns of Figs 4.3 and 4.4. Standard deviations correspond to the averaging of four quadrants by 'symmetrization' of the raw data. ${ }^{2} D_{3 / 2}^{\circ}$-state signal is inverted for comparison to ${ }^{2} S_{1 / 2}^{o}$-state signal. 


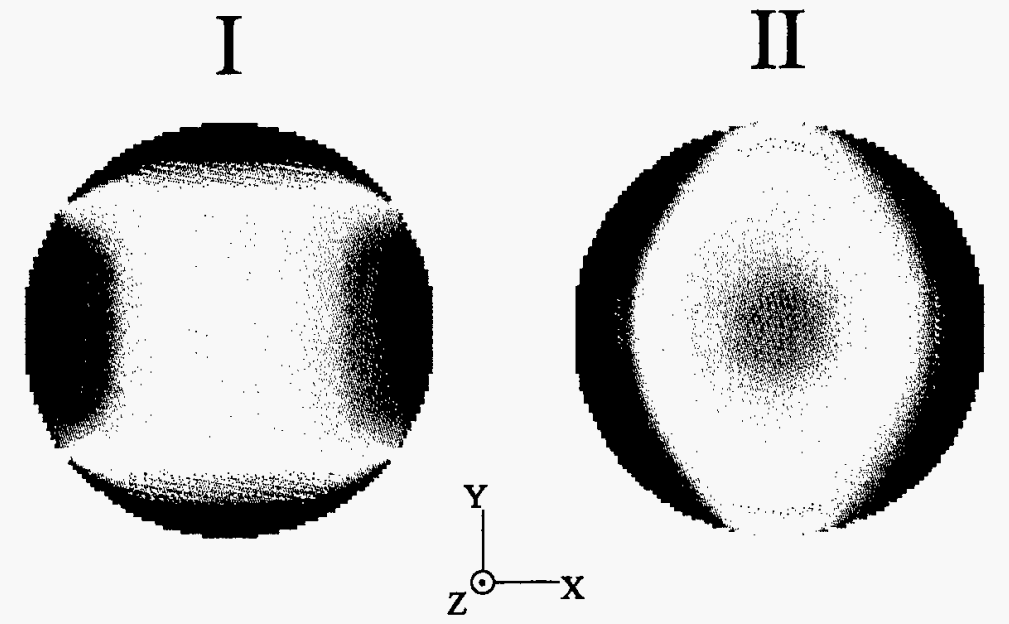

Figure 4.9: (color) Simulation of alignment images for Dissociation Geometries I and II (compare to Figs 4.3 and 4.4). These simulations were obtained by setting $s_{2}=2 \alpha_{2}$ (corresponding to a perpendicular transition) and $\alpha_{2} / \eta_{2}=-1.5$. It was possible to determine these relative values without knowledge of the 2 -photon linestrength factors.

linear combination of four basis images, each of which correspond to a specific alignment mechanism. We have done this for the chlorine alignment images presented above. Due to the quantitative shortcomings of the present data, we have not attempted a quantitative fit but try instead to reproduce only the coarse features of the measured images. The simulated alignment images shown in Fig. 4.9 include substantial contributions from basis images (a) and (c) of Fig. 3.3, which correspond to incoherent and coherent perpendicular excitation, respectively. This simulation was obtained by adding basis images (a) and (c) in relative amounts corresponding to a ratio of alignment anisotropy parameters $\alpha_{2} / \eta_{2}=-1.5$. [As discussed in Sec. 3.5, the relative values of $s_{2}$ and $\alpha_{2}$ were already fixed in constructing basis functions (a) and (b).] The fact that we only determine the ratio of $\alpha_{2}$ to $\eta_{2}$ is a consequence of not knowing the two-photon linestrengths. When the linestrength factors are known, this method can be used to obtain a quantitative value for each alignment anisotropy parameter.

The observed contribution from incoherent perpendicular excitation is consistent with previous investigations of chlorine dissociation $[10,11,85,88]$, although in all previous studies, the symmetry of the excitation was deduced from the velocity 
anisotropy rather than from the alignment angular distribution. The above analysis is also the first identification of a coherent contribution in the atomic alignment angular distribution. Although coherence effects have been seen before in total alignment measurements (e.g. for the calcium dimer) [47, 92], such measurements are not capable of fully separating coherent and incoherent contributions to the alignment. Instead, the sum $\alpha_{2}+\gamma_{2}+\eta_{2}$ is determined. In contrast, $\mathbf{v}-\mathbf{j}$ correlation measurements such as ours make it possible to determine each alignment anisotropy parameter individually.

At first glance, the existence of a coherent contribution to the photofragment alignment does not seem surprising and the incoherent contribution even less so. The $\widetilde{C}$-state of chlorine, which is believed to be the primary absorber, is a degenerate pair of $|\Omega|=1$ states. One might be tempted to conclude therefore, that coherent and incoherent excitation of the $\widetilde{C}$-state, followed by adiabatic dissociation, is all that is required to explain the observed alignment. As it turns out, the situation is not so simple: in fact, neither alignment contribution can be explained if the dissociation is strictly adiabatic. There is no contradiction however, since the treatment of alignment so far has only addressed the angular distribution but had nothing to say about how strong the effect is. These quantitative issues can be better understood by inspecting the detailed form of the dynamical functions, $f_{k}\left(\mathrm{q}, \mathrm{q}^{\prime}\right)$.

\subsection{A Closer Look at the Dynamical Functions: Implications for Chlorine Dissociation}

So far, the discussion of atomic polarization has centered on the role of initial state symmetries and coherence effects. We have seen that unique recoil angle dependencies exist for atomic alignment caused by parallel and perpendicular transitions, as well as by two types of coherent excitation. These qualitative features can be identified in ion image data even when the REMPI linestrength factors are unknown. At present, we are able to determine the relative sizes of the anisotropy parameters $s_{2}$ and $\alpha_{2}$ with respect to $\eta_{2}$, however we do not know their individual absolute value or their sign. 
A quantitative determination of the anisotropy parameters allows one, in principle, to deduce the relative contribution of each degenerate asymptotic state to the reaction products. Each potential energy curve correlates to a unique wavefunction in the atomic basis, which is determined by the Coulomb interaction at large interatomic separation $[45,46]$. These correlations are well-understood, and depend only on the generic quantum numbers $\left(J, \Omega, J_{a}, J_{b}, \Omega_{a}, \Omega_{b}\right)$ of the system. The present task is to describe the nature of the asymptotic states and the physical basis for their relative contribution to the final polarization, which is governed by initial absorption probabilities and nonadiabatic dynamics during the course of dissociation.

All the molecule-specific details of the dissociation dynamics are contained within the dynamical functions, $f_{k}\left(\mathrm{q}, \mathrm{q}^{\prime}\right)$. Although a great deal of theoretical work precedes it (see e.g. [51, 93, 94]), the first detailed discussion of the functions $f_{k}\left(\mathrm{q}, \mathrm{q}^{\prime}\right)$ appeared in a 1993 paper by Kuprianov and Vasyutinskii [45]. This work used a half-collision approach to derive expressions for total polarization cross sections. The expression for $f_{k}\left(\mathrm{q}, \mathrm{q}^{\prime}\right)$, which will be discussed below, is

$$
\begin{aligned}
f_{k}\left(\mathrm{q}, \mathrm{q}^{\prime}\right) & =\left[\left(2 J_{a}+1\right)(2 k+1)\right]^{-1 / 2} \\
& \times \sum_{n \Omega, n^{\prime} \Omega^{\prime}} \sum_{J_{b} \Omega_{b}} \sum_{\Omega_{a} \Omega_{a}^{\prime}} T_{J_{a} \Omega_{a} J_{b} \Omega_{b}}^{n \Omega} T_{J_{a} \Omega_{a}^{\prime} J_{b} \Omega_{b}}^{n \Omega_{b}^{\prime}} C_{J_{a} \Omega_{a}^{\prime} k\left(\mathrm{q}-\mathrm{q}^{\prime}\right)}^{J_{a} \Omega_{a}} \\
& \times \sum_{\Omega_{g}} \sum_{n_{e} n_{e}^{\prime}} \sigma\left(\Omega_{g} \rightarrow n_{e} \Omega, n_{e}^{\prime} \Omega^{\prime}\right) N_{n \Omega, n^{\prime} \Omega^{\prime}}^{n_{e} \Omega, n, \Omega^{\prime} \Omega^{\prime}} \delta_{\Omega-\Omega_{g}, \mathrm{q}} \delta_{\Omega^{\prime}-\Omega_{g}, \mathbf{q}^{\prime}}
\end{aligned}
$$

with quantum numbers defined as follows:

$$
\begin{gathered}
\Omega_{g}-\text { ground state angular momentum projection } \\
\Omega, \Omega^{\prime}-\text { excited state angular momentum projection } \\
n, n^{\prime}-\text { excited state, other quantum numbers } \\
n_{e}, n_{e}^{\prime}-\text { excited state, other quantum numbers, F-C region } \\
J_{a}, \Omega_{a}, \Omega_{a}^{\prime}-\text { angular momentum and its projection for atom } \mathrm{A} \\
J_{b}, \Omega_{b}-\text { angular momentum and its projection for atom } \mathrm{B} .
\end{gathered}
$$

Primed quantum numbers allow for coherence effects and do not appear for the unobserved atom $\mathrm{B}$. The quantum numbers $n_{e}, n_{e}^{\prime}$ describe the molecular system in the 
Franck-Condon region prior to nonadiabatic interactions, and the analogous $\Omega_{e}, \Omega_{e}^{\prime}$ are not needed if we neglect $\Omega$-changing nonadiabatic transitions induced by Coriolis interactions. The two $\delta$-functions connect transition moment projections to angular momentum projections, thus specifying the excited state symmetry(s). $C_{J_{a} \Omega_{a}^{\prime} k\left(\mathrm{q}-\mathrm{q}^{\prime}\right)}^{J_{a} \Omega_{a}}$ is a Clebsch-Gordan coefficient [73, 95].

The remaining factors in Eq. (4.3) contain the expected dynamics. $\sigma\left(\Omega_{g} \rightarrow\right.$ $\left.n_{e} \Omega, n_{e}^{\prime} \Omega^{\prime}\right)$ is an absorption cross section, including the primed state to allow for coherent excitation. The ultimate targets of a quantitative polarization measurement are values for these cross sections and for the nonadiabatic transition probabilities $N_{n \Omega, n^{\prime} \Omega^{\prime}}^{n_{e} \Omega, n^{\prime} \Omega^{\prime}}$. While there is no guarantee that a unique set of these quantities can be determined from a given measured polarization, Eq. (4.3) brings us much further down the road to disentangling the dissociation dynamics of simple molecules.

Finally, the expansion coefficients $T_{J_{a} \Omega_{a} J_{b} \Omega_{b}}^{n \Omega}$ describe the molecular system at large internuclear distance in terms of atomic basis functions:

$$
|n, \Omega\rangle_{\hat{A}} \stackrel{R \rightarrow \infty}{\longrightarrow} \sum_{\substack{J_{a} \Omega_{a} \\ J_{b} \Omega_{b}}} T_{J_{a} \Omega_{a} J_{b} \Omega_{b}}^{n \Omega}\left|J_{a} \Omega_{a}\right\rangle\left|J_{b} \Omega_{b}\right\rangle
$$

where $\hat{A}$ indicates that the wavefunction is antisymmetrized. As the separation between the recoiling atoms increases, the exchange interaction rapidly approaches zero so that only the Coulomb interaction remains, and the asymptotically degenerate states are described by Eq. (4.4). The coefficients $T_{J_{a} \Omega_{a} J_{b} \Omega_{b}}^{n \Omega}$ are obtained from a degenerate perturbation theory calculation [45]. Mathematically, the effect of longrange interactions on atomic polarization is simply a consequence of the symmetry of the asymptotic states. Physically, one may imagine the different asymptotic states corresponding to pairs of charge distributions with different relative orientations.

Vasyutinskii's 1981 theoretical paper on atomic photofragment orientation [94] first addressed the role of the coefficients $T_{J_{a} \Omega_{a} J_{b} \Omega_{b}}^{n}$ in atomic polarization, though a more complete discussion of the underlying physics appears in Refs $[45,46]$. A very recent manuscript [46] provides further details for all possible combinations of two recoiling atoms with $J= \pm 1 / 2$ and $\pm 3 / 2$. When at least one of the two atoms has $J=1 / 2$, then only dipole-dipole interactions are important. Molecular states from 
Table 4.1: Molecular wavefunctions and relative energies for quadrupole-quadrupole interatomic interaction. Reproduced from Ref. [45]

\begin{tabular}{cll}
\hline $\begin{array}{c}\text { Relative } \\
\text { energy }\end{array}$ & $\begin{array}{l}\text { Molecular State } \\
|\Omega, x\rangle\end{array}$ & $\begin{array}{l}\text { Expansion of wavefunctions }|\Omega, x\rangle \\
\text { in atomic basis }\left|3 / 2 \Omega_{a} ; 3 / 2 \Omega_{b}\right\rangle\end{array}$ \\
\hline 7 & $|2, b\rangle$ & $\frac{1}{\sqrt{2}}(|3 / 2,3 / 2 ; 3 / 2,1 / 2\rangle+|3 / 2,1 / 2 ; 3 / 2,3 / 2\rangle)$ \\
4 & $|1, c\rangle$ & $\frac{1}{\sqrt{2}}(|3 / 2,3 / 2 ; 3 / 2,-1 / 2\rangle-|3 / 2,-1 / 2 ; 3 / 2,3 / 2\rangle)$ \\
2 & $\left|0^{-}, b\right\rangle$ & $\sum_{\Omega_{a} \Omega_{b}}\left(\frac{2}{\sqrt{5}} C_{3 / 2 \Omega_{a} 3 / 2 \Omega_{b}}^{10}-\frac{1}{\sqrt{5}} C_{3 / 2 \Omega_{a} 3 / 2 \Omega_{b}}^{30}\right)\left|3 / 2, \Omega_{a} ; 3 / 2, \Omega_{b}\right\rangle$ \\
2 & $|1, b\rangle$ & $\frac{1}{\sqrt{2}}(|3 / 2,3 / 2 ; 3 / 2,-1 / 2\rangle+|3 / 2,-1 / 2 ; 3 / 2,3 / 2\rangle)$ \\
0 & $\left|0^{+}, b\right\rangle$ & $\sum_{\Omega_{a} \Omega_{b}} C_{3 / 2 \Omega_{a} 3 / 2 \Omega_{b}}^{00}\left|3 / 2, \Omega_{a} ; 3 / 2, \Omega_{b}\right\rangle$ \\
-1 & $|2, a\rangle$ & $\frac{1}{\sqrt{2}}(|3 / 2,3 / 2 ; 3 / 2,1 / 2\rangle-|3 / 2,1 / 2 ; 3 / 2,3 / 2\rangle)$ \\
-3 & $|1, a\rangle$ & $|3 / 2,1 / 2 ; 3 / 2,1 / 2\rangle$ \\
-3 & $|3\rangle$ & $|3 / 2,3 / 2 ; 3 / 2,3 / 2\rangle$ \\
-6 & $\left|0^{+}, a\right\rangle$ & $\sum_{\Omega_{a} \Omega_{b}} C_{3 / 2 \Omega_{a} 3 / 2 \Omega_{b}}^{20}\left|3 / 2, \Omega_{a} ; 3 / 2, \Omega_{b}\right\rangle$ \\
-8 & $\left|0^{-}, a\right\rangle$ & $\sum_{\Omega_{a} \Omega_{b}}\left(\frac{1}{\sqrt{2}} C_{3 / 2 \Omega_{a} 3 / 2 \Omega_{b}}^{10}+\frac{2}{\sqrt{5}} C_{3 / 2 \Omega_{a} 3 / 2 \Omega_{b}}^{30}\right)\left|3 / 2, \Omega_{a} ; 3 / 2, \Omega_{b}\right\rangle$
\end{tabular}

atoms of like parity are distinguished only by second order perturbation theory (van der Waals interaction), while those from atoms of unlike parity appear already in first order. When both atoms have $J=3 / 2$, quadrupole-quadrupole interactions dominate. For the purpose of discussing chlorine dissociation, Table I of Ref. [45] is reproduced here as Table 4.1. This table provides the correlations between molecular and atomic states when two $J=3 / 2$ atoms are involved.

A quantitative measurement of atomic alignment should make it possible to determine the relative contribution of each asymptotic state, however, we may begin to predict the range of possibilities by considering a simplified case. Since chlorine dissociation at $355 \mathrm{~nm}$ occurs primarily through a perpendicular transition to produce two ground state atoms, it is reasonable as a starting point to consider only the states $| \pm 1, a\rangle,| \pm 1, b\rangle$ and $| \pm 1, c\rangle$ of Table 4.1. We would like to know which of these will to contribute to atomic alignment through either a coherent or incoherent mechanism. It 
is instructive to write the molecular-frame density matrix for $J=3 / 2$ atoms produced by dissociation along $\Omega= \pm 1$ states. Consider a general pure state of the form

$$
\left.\left.\left.\left.\left.\left.\left.\left.\left.\left.\left.\left.|\psi\rangle=\left.A\right|^{+\frac{3}{2}}\right\rangle\left.\right|^{-\frac{1}{2}}\right\rangle+\left.B\right|^{-\frac{3}{2}}\right\rangle\left.\right|^{+\frac{1}{2}}\right\rangle+\left.C\right|^{+\frac{1}{2}}\right\rangle\left.\right|^{-\frac{3}{2}}\right\rangle+\left.D\right|^{-\frac{1}{2}}\right\rangle\left.\right|^{+\frac{3}{2}}\right\rangle+\left.E\right|^{+} \frac{1}{2}\right\rangle\left.\right|^{+\frac{1}{2}}\right\rangle+\left.F\right|^{-\frac{1}{2}}\right\rangle\left.\right|^{-\frac{1}{2}}\right\rangle,
$$

where $\left|\Omega_{a}\right\rangle\left|\Omega_{b}\right\rangle \equiv\left|J_{a} \Omega_{a}\right\rangle\left|J_{b} \Omega_{b}\right\rangle$. For adiabatic dissociation, the coefficients $A-F$ are simple products of coefficients $T_{J_{a} \Omega_{a} J_{b} \Omega_{b}}^{n \Omega}$, but are more complex sums of such products when multiple excitations or nonadiabatic transitions are involved. The density matrix for the space $\left|\Omega_{a}\right\rangle$ can be calculated from the expression $\rho_{\Omega_{a} \Omega_{a}^{\prime}}=$ $\sum_{\Omega_{b}}\left\langle\Omega_{b}\left|\left\langle\Omega_{a}|\hat{\rho}| \Omega_{a}^{\prime}\right\rangle\right| \Omega_{b}\right\rangle$ from Ref. [74], where the operator $\hat{\rho}=|\psi\rangle\langle\psi|$. The resulting matrix is

$$
\boldsymbol{\rho}_{\Omega_{a} \Omega_{a}^{\prime}}=\left(\begin{array}{cccc}
A^{2} & \cdot & B E^{*} & \cdot \\
\cdot & F^{2}+D^{2} & \cdot & F A^{*} \\
B^{*} E & \cdot & E^{2}+C^{2} & \cdot \\
\cdot & F^{*} A & \cdot & B^{2}
\end{array}\right),
$$

where the columns $\left(\Omega_{a}^{\prime}\right)$ and rows $\left(\Omega_{a}\right)$ correspond from the top left to $-3 / 2,-1 / 2$, $1 / 2$ and $3 / 2$.

We can now draw conclusions about the alignment contribution of the states in Table 4.1 by looking at where their coefficients appear in the above density matrix. Either pair of states $| \pm 1, b\rangle$ or $| \pm 1, c\rangle$ (which contain coefficients $A-D$ ) contribute equally to each of the diagonal elements, corresponding to equal population of all states $\Omega_{a}$ and no alignment. Furthermore, although both degenerate components $\Omega= \pm 1$ of these two states are excited with equal probability, the absence of $A-D$ (squared) in the off-diagonal matrix elements indicates that adiabatic dissociation on $| \pm 1, b\rangle$ or $| \pm 1, c\rangle$ does not contribute to observable coherence effects. On the other hand, excitation of the states $| \pm 1, a\rangle$ (coefficients $\mathrm{E}, \mathrm{F}$ ) leads to population in $\Omega_{a}= \pm 1 / 2$, corresponding to cylindrically symmetric alignment. Finally, the only coherent (off-diagonal) contribution comes from asymptotic dissociation along the states $| \pm 1, b\rangle$ or $| \pm 1, c\rangle$ and the states $| \pm 1, a\rangle$.

Although chlorine is widely believed to dissociate adiabatically, the above analysis suggests that this is unlikely. Comparison of the energy ordering in $\mathrm{Ta}$ ble 4.1 with that of the known $\Omega=1$ states in $\mathrm{Cl}_{2}$ shows that the $\mathrm{Cl}_{2}\left(\widetilde{C}^{1} \Pi_{1 u}\right)$-state 


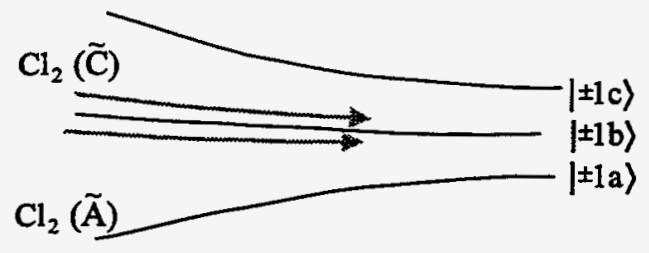

(a)

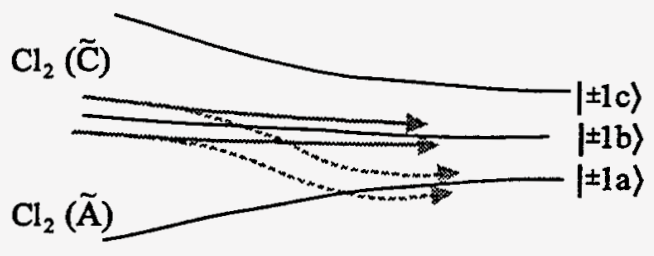

(b)

Figure 4.10: (a) Despite coherent excitation of the states $| \pm 1, b\rangle$, there will be no observable alignment (coherent or incoherent) without nonadiabatic transitions. (b) A nonadiabatic transition to states $| \pm 1, a\rangle$ is required to observe either type of alignment effect. An observable coherence effect requires substantial asymptotic 'population' in both $| \pm 1, a\rangle$ and $| \pm 1, b\rangle$ (or $| \pm 1, a\rangle$ and $| \pm 1, c\rangle$ ).

corresponds to $| \pm 1, b\rangle$. Thus, if dissociation occurred exclusively on the $\tilde{C}$-state, we would see neither coherent nor incoherent contributions to alignment. One may also reasonably eliminate the possibility that the alignment is caused by a second absorber. While the $\tilde{B}^{3} \Pi_{0_{u}^{+}}$-state is known to contribute at least a few percent to the absorption probability $[82,85,88]$, it has the wrong symmetry $(\Omega=0)$ to explain the observed coherence effect. The only state of the proper symmetry is $\tilde{A}^{3} \Pi_{1 u}$, which corresponds to $| \pm 1, a\rangle$. While this state would be expected to cause alignment, and the Franck-Condon region is believed to be close to that of the $\widetilde{C}$-state, its absorption cross section is at least 100 times less [96]-too small to explain the observed alignment effect.

Unless there is a serious (and unlikely) misunderstanding of the electronic structure of the chlorine molecule, nonadiabatic transitions are the only reasonable explanation for the observed alignment effect. Although there are no known avoided crossings in the regions of interest, transitions between asymptotically degenerate states are quite possible (Fig. 4.10). Since the $\widetilde{C}$-state $(| \pm 1, b\rangle)$ is almost certainly the primary absorber, an asymptotic transition $| \pm 1, b\rangle \longrightarrow| \pm 1, a\rangle$ is necessary to give both the coherent and incoherent alignment contributions. A nonadiabatic transition to $| \pm 1, c\rangle$ may also contribute to both sources of alignment, but this is not required. 


\subsection{Polarized $\mathrm{O}\left({ }^{1} D_{2}\right)$ from the Dissociation of Ozone at $266 \mathrm{~nm}$.}

The theoretical problem of describing atomic photofragment polarization has been largely solved for dissociations of diatomic molecules. The next major theoretical challenge will be to treat polyatomic molecules. One would expect that the general description of atomic polarization will remain quite similar. It will still be true that state symmetries, coherence effects, nonadiabatic effects and long-range interactions will govern the polarization. Of these, a proper description of the long-range interactions appears to be the most immediate challenge-the effects of a vibrating and rotating diatomic partner are likely to be much more complex than for an atomic partner. It also seems plausible that internal motion of the partner will have an important role at short range, and that the phase of this motion with respect to the position of the departing atom might also be significant.

Experimentalists who are aware of the state of the art in the theory of atomic photofragment polarization (or doing theory themselves) have typically studied diatomic dissociations, for the likely reason that they hoped to be able to interpret their measurements. In contrast, there have been several recent observations of the atomic $\mathbf{v}$-J correlation by REMPI-equipped investigators who practically stumbled upon the effect while studying more conventional aspects of polyatomic dissociations. Although so far it has not been possible to provide a rigorous analysis of the results, these studies have made the field visible to a wider audience and provided stimulus for theoretical work on polyatomic systems. At the time of this writing, Suzuki and Vasyutinskii are collaborating on the analysis of polarized $S\left({ }^{1} D_{2}\right)$ produced by the dissociation of OCS [54], and on the polyatomic problem in general. Those of us who are newcomers to this field hope that theoretical progress in the next few years will make it possible to use atomic polarization as a diagnostic tool for determining excited state symmetries and nonadiabatic transition probabilities in polyatomic dissociations.

The earliest report of the atomic $\mathbf{v}-\mathbf{J}$ correlation in any molecular dissoci- 
ation was for $\mathrm{O}\left({ }^{1} D_{2}\right)$ produced in the $205 \mathrm{~nm}$ dissociation of ozone [52]. In that single-color ion imaging experiment, the atomic oxygen images showed a characteristic 'hole' at the poles of an otherwise positive- $\beta$ angular distribution, demonstrating the existence of a $\mathbf{v}-\mathbf{J}$ correlation. That work was the stimulus for our further study of ozone at $266 \mathrm{~nm}$. While this wavelength is of no greater interest from the perspective of ozone reaction dynamics, the absorption cross section is much larger, and the ability to independently rotate the REMPI probe laser polarization in a two-laser experiment makes it more straightforward to study the $\mathbf{v}-\mathbf{J}$ correlation. Most important for our purposes, the coarse features of the dissociation dynamics are reasonably well-known, allowing new studies to concentrate on polarization effects.

The near ultraviolet absorption spectrum and relevant potential energy curves for ozone are shown in Fig. 4.11. The Hartley band is a strongly absorbing continuum which peaks in the mid-200 nm region. The primary absorber is thought to be the $\widetilde{D}^{1} A^{\prime}$ state ( ${ }^{1} B_{2}$ in $C_{2 v}$ symmetry), which correlates adiabatically to singlet products. At $266 \mathrm{~nm}$, roughly $10 \%$ of the products are in the triplet channel, which is reached by a nonadiabatic transition at the avoided crossing between the $\widetilde{D}$ and $\widetilde{C}$ states. At wavelengths below $200 \mathrm{~nm}$, absorption to the $\widetilde{R}^{1} A^{\prime}$ state $\left({ }^{1} B_{2}\right.$ in $\mathrm{C}_{2 \mathrm{v}}$ ) begins to dominate. The structure to the long-wavelength side (known as the Huggins band) is due to bound or quasi-bound portions of the $\widetilde{D}$-state and to a lesser extent from the weakly-absorbing $\widetilde{C}^{1} A^{\prime}$ state $\left({ }^{1} A_{1}\right.$ in $\left.C_{2 v}\right)$, which correlates to ground state products. These assignments have been deduced from measurements of recoil kinetic energy [97-99], quantum-state-specific detection of both singlet and triplet products [100-102], and theoretical work [103, 104]. These interpretations are also consistent with the extensive recent $a b$ initio electronic structure calculations of Banichevich et al. $[105,106]$.

There remains little mystery about the coarse features of ozone dissociation dynamics at $266 \mathrm{~nm}$-absorption is dominated by one electronic state of known symmetry, which correlates adiabatically (at least until the asymptotic region) to the singlet products which we detect. For this reason, it is a desirable system for testing new theories on atomic photofragment polarization. It remains to be seen whether the detailed motions in the bonding region of the potential energy surface 

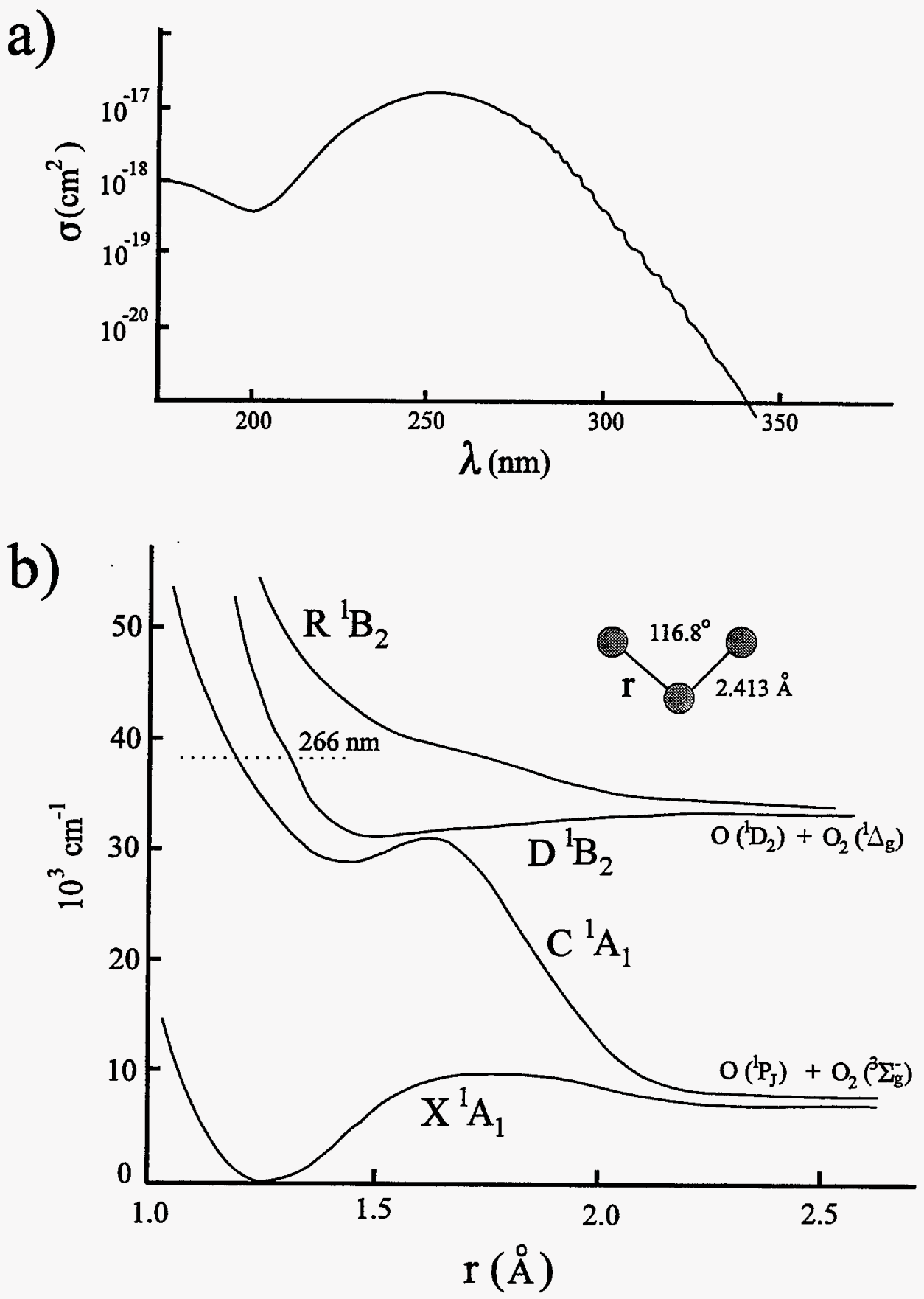

Figure 4.11: Ozone near ultraviolet absorption spectrum (a) and relevant potential energy surfaces (b). The curves in (b) are for the removal of one oxygen atom with the other atoms fixed in the geometry shown. 
have an influence on atomic polarization - especially whether such detailed effects will be observable in the recoil kinetic energy dependence of the atomic polarization.

Most of the experimental details for these ozone experiments have been discussed in previous sections. Briefly, the fourth harmonic of a Nd:YAG laser (266 nm, $0.5 \mathrm{~mJ}$ ) dissociated a fraction of the ozone molecules in a skimmed supersonic expansion (20\% in helium). After a 20 ns delay, the $O\left({ }^{1} D_{2}\right)$ photofragments were detected using 2+1 REMPI through either of two autoionizing resonances [203.6 nm or $205.4 \mathrm{~nm}$ (see Fig. 2.4)]. Ion image data were collected in an apparatus very similar to that described in Sec. 2.3. As usual, images were collected with the probe laser polarized along both the $Y$ and $Z$ axes, but only Geometry II $\left(\mathbf{e}_{d i s s} \| \mathbf{Y}\right)$ was used for dissociation.

Fig. 4.12 shows symmetrized image data for both probe geometries and both $2+1$ REMPI states. The effect of angular momentum alignment is seen very clearly in these images as the modulation which depends on probe laser polarization or REMPI transition. The effect is more pronounced than for chlorine, largely because oxygen atoms have no nuclear spin which can depolarize the alignment. We have not yet been able to subtract images for the two probe polarizations, since no total alignment measurement has been done to calibrate the relative integrated intensities of each image. (At the time these image data were collected, we were not yet aware of the need for a total alignment measurement.)

The rings in these images correspond to the vibrational states $(v=0-3)$ of the $\mathrm{O}_{2}\left({ }^{1} \Delta_{g}\right)$ partner fragment. The energy of the dissociation photon is only $5490 \mathrm{~cm}^{-1}$ above the threshold for singlet products. The vibrational level spacings of $\mathrm{O}_{2}\left({ }^{1} \Delta_{g}\right)$ $\left(\omega_{e}=1509 \mathrm{~cm}^{-1}\right)$ are easily resolved, since they are a substantial fraction of this excess energy. Fig. 4.13(b) shows the radial distribution of REMPI ion signal. This distribution was obtained by integrating the inverse-Abel-transformed images [Fig. 4.13(a)] over the angle $\phi$. The fact that the shape of this distribution depends on the REMPI probe transition suggests that the degree of total alignment depends on the $\mathrm{O}_{2}$ partner vibrational state. In order to obtain a true velocity distribution, one would need to correct for these effects.

The observation of an $\mathrm{O}\left({ }^{1} \mathrm{D}_{2}\right)$ alignment effect is already interesting from the 


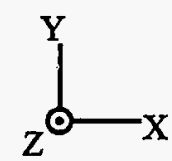

${ }^{1} \mathrm{P}_{1}$

${ }^{1} \mathrm{~F}_{3}$

Probe
Z

Probe
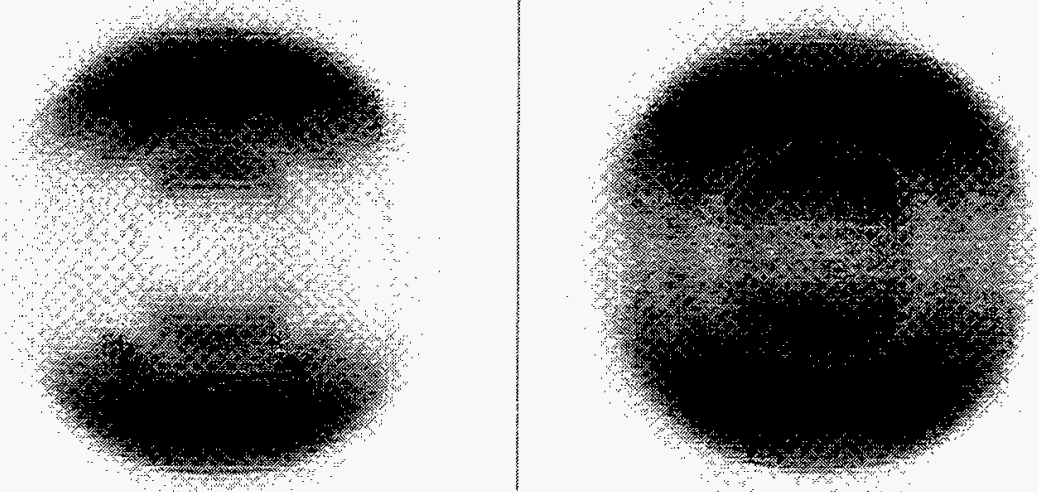

Figure 4.12: Image data (symmetrized) for ozone dissociation (Geometry II: $\mathbf{e}_{\text {diss }} \| \mathbf{Y}$ ) at $266 \mathrm{~nm}$. Two REMPI transitions (see Fig. 2.4) and both probe geometries were used. 
a)
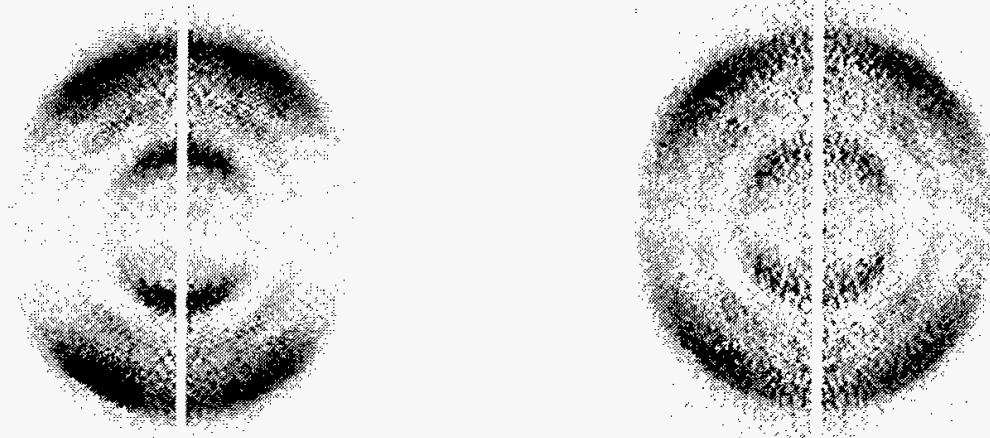

${ }^{1} \mathrm{P}_{1}$

${ }^{1} \mathrm{~F}_{3}$

b)

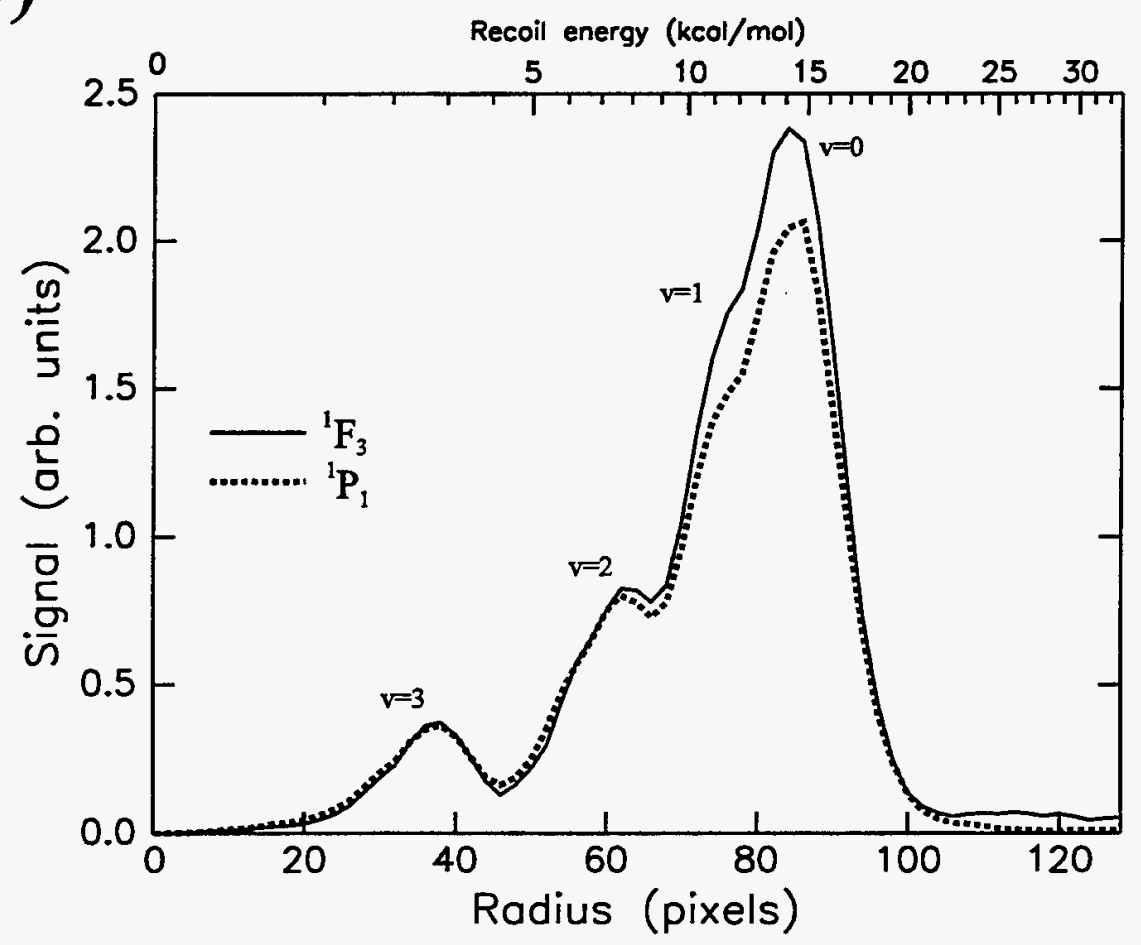

Figure 4.13: Radial distribution of ozone ion signal (b) for both REMPI transitions, obtained from inverse-Abel-transform of the data images with the probe polarization along $Y(\mathrm{a})$. (Distributions in (b) have been smoothed to highlight coarse features.) 
perspective of testing new theories on atomic polarization. The even more intriguing possibility, suggested by the radial distributions, is that the polarization depends on the vibrational state of the $\mathrm{O}_{2}$ fragment. We investigated this possibility further by radially integrating the inverse-Abel-transformed images to obtain an ion angular distribution. Fig. 4.14 shows that these angular distributions do depend on vibrational state, suggesting an energy-dependent $\mathbf{v}-\mathbf{J}$ correlation. There is reason for caution in this interpretation, however, as this dependence may be explained in two other ways. The most likely alternative is that the limited spatial resolution of the images causes proportionally larger distortions to the smaller rings of the images, changing the apparent anisotropy for smaller recoil energies. Future measurements with the new velocity-map imaging technique $[69,70]$ should help to verify or eliminate this possibility. The second alternative is that the neutral photofragment angular distribution depends on the $\mathrm{O}_{2}$ vibrational state. Of course, this would also be very interesting from the perspective of studying dissociation dynamics. Testing this possibility again requires a total alignment calibration to separate population and alignment contributions to the signal.

In conclusion, the ozone photodissociation results presented in this section show a pronounced atomic alignment effect. The resolution of several vibrational states of the $\mathrm{O}_{2}$ partner in the atomic images suggests the exciting possibility of analyzing a partner-state-resolved $\mathbf{v}-\mathbf{J}$ correlation. Angular and radial distributions of ion signal do indeed suggest that the alignment depends on recoil energy. We stress that these results are preliminary because of the limited spatial resolution and absence (so far) of a total alignment calibration. Despite these shortcomings, the data are quite striking and should provide stimulus for more experimental and theoretical work in the near future. 


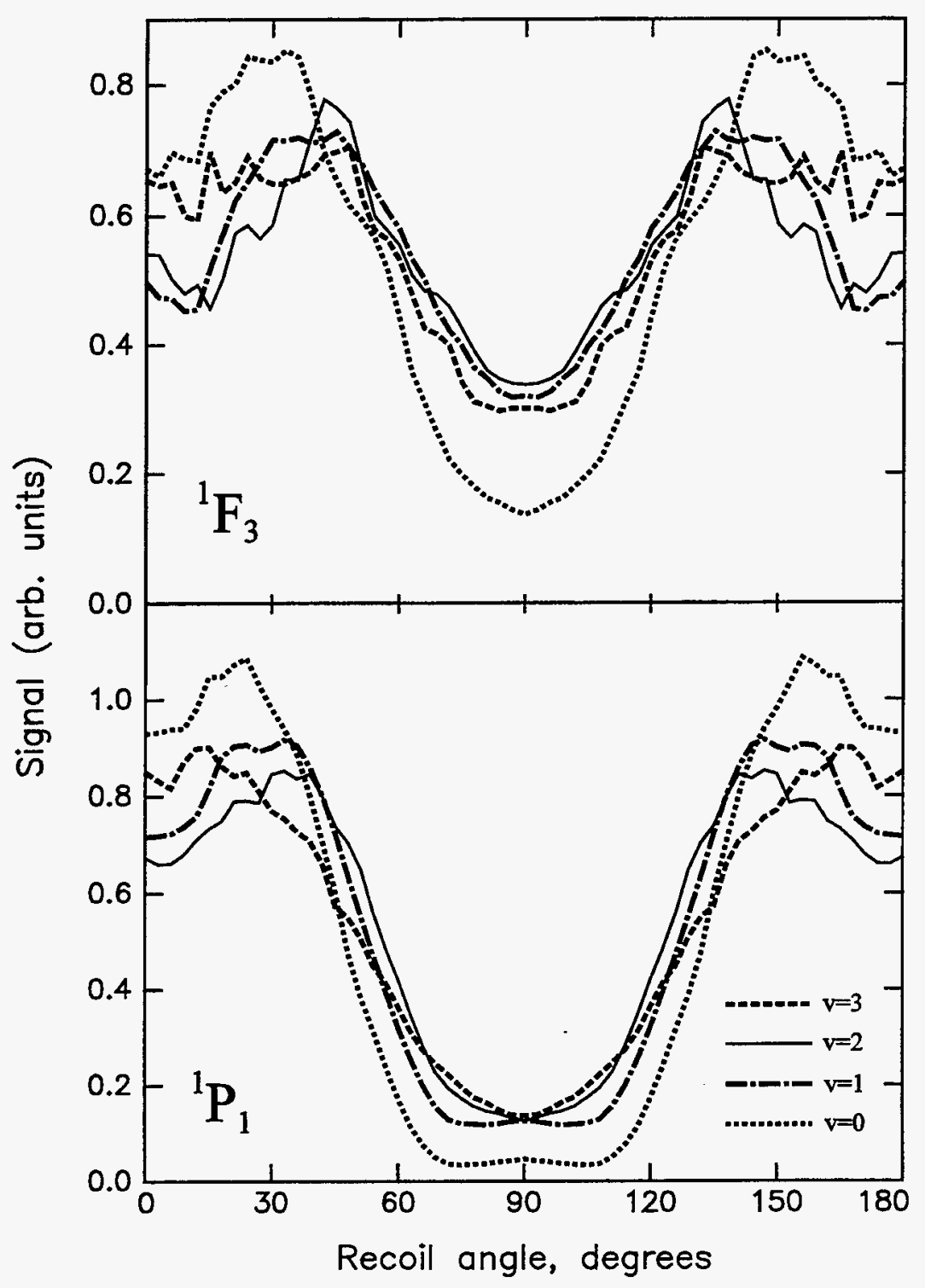

Figure 4.14: Angular distribution of ozone ion image signal for both REMPI transitions. (Smoothed to highlight coarse features.) 


\section{Chapter 5}

\section{Photochemistry of Azomethane Following Absorption of One or More Photons}

\subsection{Abstract}

We have used a form of state-selective translational spectroscopy to measure the kinetic energy release of methyl radical photofragments, which were produced in the ultraviolet dissociation of azomethane. Translational energy distributions for methyl fragments with zero and one quantum of umbrella vibration are indistinguishable within our experimental uncertainty. Compared to earlier non-state-selective measurements, these distributions show a strong preference for higher kinetic energies. Despite this difference, our observations are consistent with the previously-proposed mechanism of a concerted dissociation following randomization of internal energy. In the framework of this model, the states which we have studied are likely to be a minority product species. We have also determined that the appearance times for both methyl vibrational states are fast compared to the experimental time resolution, contradicting an earlier report. Finally, we have characterized nonresonant multiphoton processes which complicate analysis of the single-photon dissociation. 


\subsection{Introduction and Background}

Azomethane photodissociation in the near ultraviolet has recently received well-deserved attention because of the richness of the resulting three-body dissociation dynamics. The process

$$
\mathrm{CH}_{3} \mathrm{~N}_{2} \mathrm{CH}_{3} \longrightarrow 2 \mathrm{CH}_{3}+\mathrm{N}_{2}
$$

is thought to occur on the ground electronic state following internal conversion from the first excited singlet state. The central dynamical questions focus on the timing of bond breakage and the partitioning of energy between the fragments' internal modes and translation. The former question has persisted since very early studies of unimolecular dissociations $[107,108]$. From a technical perspective, studying azomethane in this wavelength region is particularly challenging because of its low absorption cross section $\left(1.9 \times 10^{-20} \mathrm{~cm}^{2}\right.$ at $\left.355 \mathrm{~nm}\right)$. This study has two goals-first, to study the detailed partitioning of energy between methyl translation and umbrella vibration, and second, to characterize the multiphoton processes for azomethane, which are difficult to avoid and may complicate data analysis when standard experimental techniques are used.

Fig. 5.1 shows schematic potential energy surfaces and the ultraviolet absorption spectrum for azomethane. The qualitative shapes of the curves are adapted from the recent $a b$ initio study by Liu et al. [109]. Photon absorption in the mid$300 \mathrm{~nm}$ region excites ground state molecules $\left({ }^{1} A_{g}\right.$ or ${ }^{~} S_{1}$ ') to the first excited singlet state $\left({ }^{1} B_{g}\right.$ or ' $S_{2}$ '). In this transition, a nitrogen non-bonding electron is promoted to an N-N pi-antibonding orbital $\left(n \rightarrow \pi^{*}\right)$. The major features of the ultraviolet absorption spectrum were assigned in spectroscopic investigations during the $1960 \mathrm{~s}$ and 70s [110-112]. Of the first four excited states (two singlet, two triplet), only the second triplet state correlates to reaction products in their ground electronic state, thus nonadiabatic processes must play a role in the production of ground state reaction products produced by dissociation around $350 \mathrm{~nm}$.

Weisman et al. $[113,114]$ initiated the present round of investigations into azomethane dissociation dynamics with their Coherent Anti-Stokes Raman (CARS) study of the methyl and nitrogen fragment appearance times and quantum state 


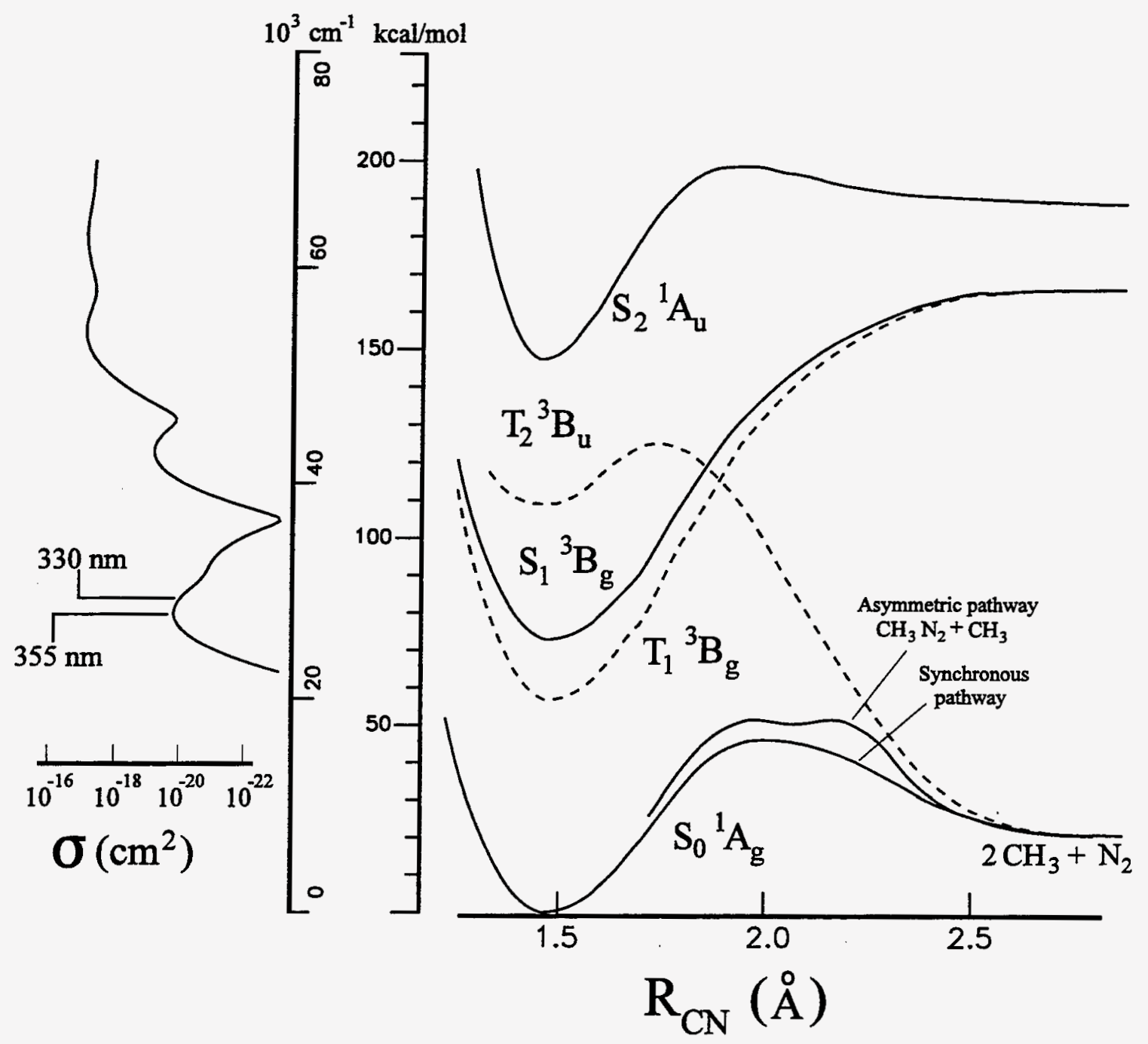

Figure 5.1: Azomethane absorption spectrum and relevant potential energy curves for symmetric stretching of both $\mathrm{C}-\mathrm{N}$ bonds (unless indicated). Around $350 \mathrm{~nm}$, the $S_{1}$ state is the primary absorber, while dissociation is believed to occur on the $S_{0}$ state following internal conversion. The absorption spectrum come from Refs [110-112] and the potential energy curves were adapted from Ref. [109]. 
populations. Their initial report [113] showed appearance times of $<1 \mathrm{~ns}$ and $5.6 \pm 1 \mathrm{~ns}$ for methyl radicals with one and zero quanta of vibration in the $\nu_{2}$ umbrella mode, respectively. The nitrogen fragment had an appearance time similar to the nonvibrating methyl. From these results they concluded that the excited azomethane molecule dissociates in a stepwise manner,

$$
\begin{aligned}
\mathrm{CH}_{3} \mathrm{~N}_{2} \mathrm{CH}_{3}^{*} & \stackrel{\leq 1 n s}{\longrightarrow} \mathrm{CH}_{3} \mathrm{~N}_{2}+\mathrm{CH}_{3} \\
\mathrm{CH}_{3} \mathrm{~N}_{2} & \stackrel{5.6 n s}{\longrightarrow} \mathrm{CH}_{3}+\mathrm{N}_{2},
\end{aligned}
$$

with the first dissociation step producing mostly umbrella-excited methyl. In their later study, they measured rotational and vibrational state distributions for the nitrogen and methyl products. Based on the umbrella-state-dependent appearance times of the earlier study, they decomposed this distribution into contributions from the first and second steps of their proposed dissociation mechanism. Their interpretation of the dissociation dynamics relied heavily on this separation. The excited umbrella vibrations of the 'first' step were seen as an indicator of rapid impulsive dissociation of excited azomethane, while the methyl rotations in $v_{2}=0$ ('second' step) and the nitrogen vibrations favored a statistical mechanism, i.e. there is sufficient time for randomization of internal energy before the methyldiazenyl radical $\left(\mathrm{CH}_{3} \mathrm{~N}_{2}\right)$ dissociates.

A different interpretation emerged from the work of North et al. [115], who used photofragment translational spectroscopy to measure recoil energy and angular distributions of methyl and nitrogen products. Three key experimental results supported this new interpretation, with the first two contradicting the interpretation (and some of the results) of Weisman et al. First, the overall velocity anisotropy was found to be zero, within experimental uncertainty. While this could be explained by a recoil vector near the 'magic angle' $\left(54.7^{\circ}\right)$ with respect to the transition moment, it is also consistent with a long-lived azomethane excited state. Second, the angular distribution for methyl radicals from the methyldiazenyl dissociation (in the methyldiazenyl center-of-mass frame) was peaked in the backward direction. This strong correlation between first and second step recoil velocity vectors indicates rapid secondary dissociation. The key conclusion from this observation is that the process 
of losing two methyl radicals cannot be stepwise, since the methyldiazenyl radical survives for less than a rotational period ( $\sim 1 \mathrm{ps})$. When considered together, the angular distributions support the assignment of statistical and rapid dissociations to the first and second steps, respectively, which is the reverse of the assignment by Weisman et al.

The third important observation of this work was of a bimodal translational energy distribution for the methyl fragment. While it was possible to fit the data by assuming that either component corresponds to the primary dissociation event, only one possibility provides a clear dynamical interpretation, namely, the low energy component goes with the first step. The following mechanism is consistent with all of these observations: Following the optical excitation $S_{0} \rightarrow S_{1}$, rapid internal conversion places the molecule in a highly vibrationally excited state of the $S_{0}$ potential energy surface. After a time longer than the azomethane rotational period, enough energy flows into one $\mathrm{CN}$ bond for the first methyl to surmount a large barrier $(\sim 50 \mathrm{kcal} / \mathrm{mol})$ with little or no exit barrier. The remaining methyldiazenyl radical, bound by less than $2 \mathrm{kcal} / \mathrm{mol}$, rapidly dissociates and the second methyl and nitrogen molecule are repelled by a large exit barrier $(25-30 \mathrm{kcal} / \mathrm{mol})$. In this type of process, which is described as 'concerted', the transition states for the loss of both methyls lie close together on the potential energy surface. In contrast, for a stepwise process, the primary fragments are separated by many molecular diameters before the secondary dissociation occurs. A concerted process lies between this and the opposite extreme of a 'synchronous' dissociation with a symmetric transition state. A two-dimensional potential energy contour map in Fig. 5.2 makes these distinctions clear. Operationally, synchronous and concerted are distinguished from stepwise by comparing the lifetime of the intermediate to its rotational period.

Neither of the above experiments permit a direct determination of which electronic state the dissociation proceeds from. While the $S_{1}$ state is accessed by optical excitation, it correlates to electronically excited fragments which are not energetically accessible at $355 \mathrm{~nm}$. Two possibilities remain-internal conversion to the ground state potential surface (already discussed), or some involvement of the lowest triplet state ${ }^{3} B_{g}$ (' $T_{1}$ '). Liu et al. recently reported an $a b$ initio electronic structure 

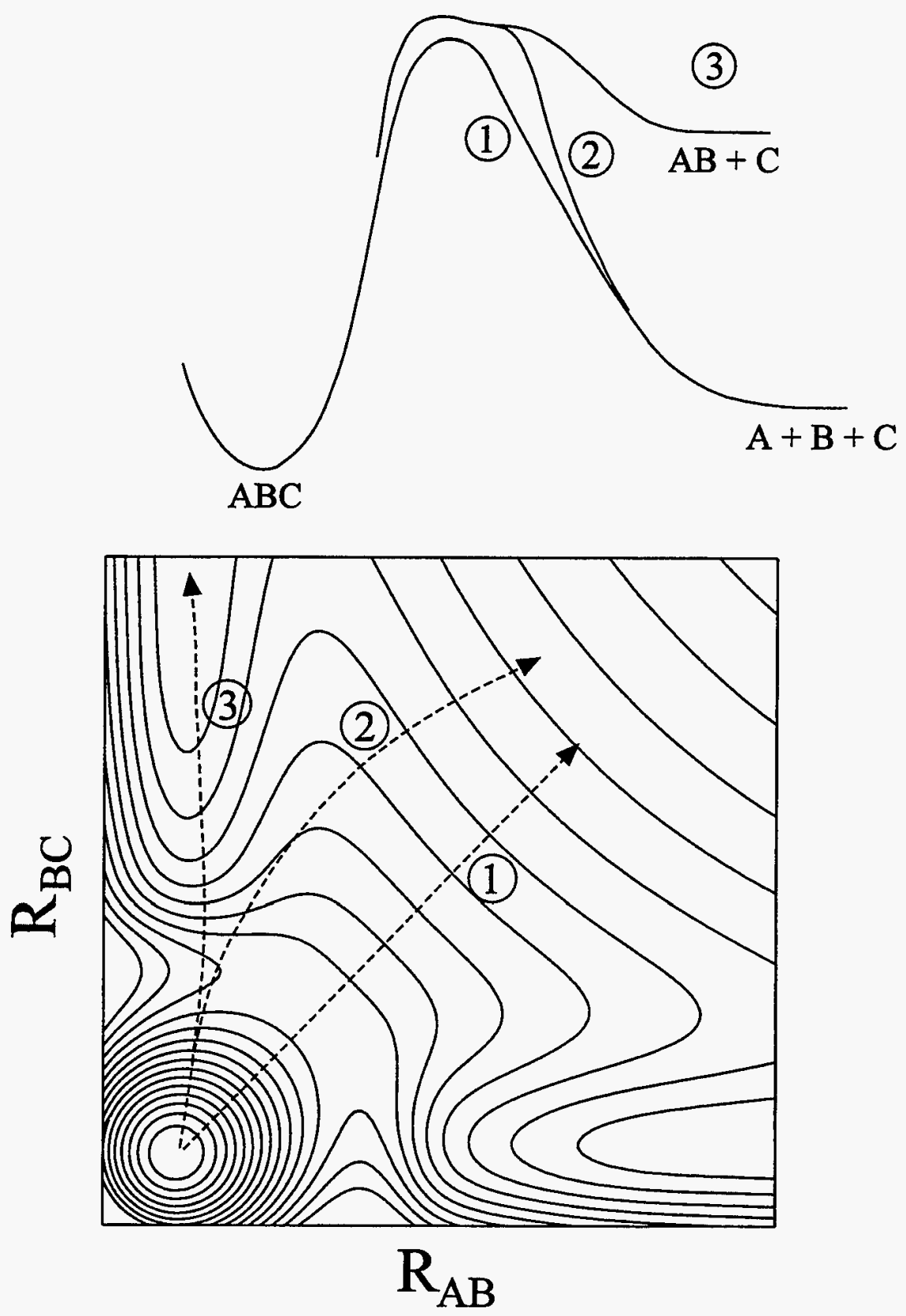

Figure 5.2: Synchronous, concerted, and stepwise trajectories on a potential energy contour map. (1) Synchronous/symmetric. (2) Concerted. (3) Stepwise (dissociates at large $\mathrm{B}-\mathrm{C}$ separation, if at all. 
<smiles>CN=NC</smiles>

$\mathrm{S}_{1}$<smiles>CN=NC</smiles>

$\mathrm{S}_{1} \rightarrow \mathrm{S}_{0}$

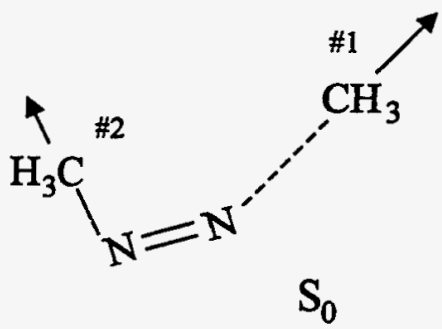

Figure 5.3: Dissociation mechanism proposed by Liu et al. [109] and by North et al. [115] and consistent with measurements of North. Azomethane is optically excited to the $S_{1}$ electronic state. Torsion and CNN bend bring $S_{1}$ and $S_{0}$ surfaces close together in energy, permitting efficient internal conversion. Following statistical randomization of internal energy, molecule dissociates with an asymmetric transition state.

study to address these and other questions related to the dissociation dynamics. Their proposed dissociation mechanism is indicated schematically in Fig. 5.3. They found that a combination of increasing CNN angle and CNNC torsion brings the $S_{0}$ and $S_{1}$ surfaces within only a few $\mathrm{kcal} / \mathrm{mol}$ of each other. Since the internal conversion rate depends roughly on the inverse of the energy gap, this is a likely candidate for the nonadiabatic process which allows dissociation to proceed. In contrast, accessing the triplet state requires either a low-probability intersystem crossing from $S_{1}$ or spin-forbidden optical transition.

The detailed features of the $S_{1}$ surface calculated by Liu et al. go a long way in explaining the subsequent dynamics. On the region of $S_{1}$ where internal conversion occurs, the minimum energy path is in the torsional coordinate. Since this is orthogonal to the dissociation coordinate, motion along this minimum initiates scrambling of the vibrational energy and is thus consistent with a long dissociation lifetime. Surprisingly at first, the synchronous (symmetric) dissociative pathway was found to be $7 \mathrm{kcal} / \mathrm{mol}$ lower than the concerted path, but since the synchronous path is such a small region of configuration space, the concerted mechanism is favored when free energy is considered. Finally, the calculated barrier to methyldiazenyl dissociation was found to be only $0.2 \mathrm{kcal} / \mathrm{mol}$.

While the $a b$ initio results clearly favor the interpretation of North $e t$ al., the disagreement with Weisman has not been resolved. In order to solidify the present interpretation, a logical next step is to measure correlated translational-internal en- 
ergy distributions. For example, it would be interesting to study the partitioning of internal states between components of the bimodal translational energy distribution. A recent REMPI-TOFMS study by Fairbrother et al. [116] has made some progress in this direction. Their results suggest that vibrationless methyl radicals are present only in the fast component of the translational energy distribution. This surprising result suggests that either the translational-vibrational partitioning is highly state specific, or that the low-energy component measured by North et al. may be an artifact. Although no results were shown, they reported that umbrella-excited methyls showed similar TOFMS profiles.

A great deal has already been learned about the azomethane system, but as a prototype for three-body dissociation of symmetric molecules, the picture remains naggingly incomplete. In particular, the disagreement between the results of Weisman et al. and North et al. has not been resolved. The correlated measurements of Fairbrother et al. are intriguing and should be pursued further. Furthermore, the high laser power densities which one is tempted to use for a low-signal experiment present special complications, which may have affected both of the state-resolved experiments mentioned so far. In undertaking a new state-resolved measurement, we have characterized these complications and accounted for them in our data analysis, while clarifying promising approaches for additional investigations in the future.

\subsection{Experimental Methods}

A pulsed supersonic molecular beam of $10 \%$ azomethane in helium was expanded into a differentially pumped source chamber and skimmed prior to entering the interaction region of the experiment. Although no explicit additional measures were taken to eliminate clustering, the experiment was carried out under conditions which were characterized in a previous study to have negligible cluster contributions. We confirmed a clear onset of cluster contributions in the mass spectrum and TOFMS peak profile shapes at least 50 microseconds later in the gas pulse than our standard operating conditions. The molecular beam was crossed within a set of ion extraction optics by one or more lasers. In the two-laser experiment, the photolysis laser was 
the $8 \mathrm{~ns}$ pulse of the third harmonic of a Nd:YAG laser $(355 \mathrm{~nm})$, and the probe pulse at around $330 \mathrm{~nm}$ was the doubled output of an Nd:YAG-pumped dye laser. Typical laser pulse energies were $8 \mathrm{~mJ}$ and $3 \mathrm{~mJ}$ for dissociation and probe lasers, respectively. In a second set of measurements, a single laser was used to study the dissociation process at the wavelength of the probe laser.

The focussing conditions for the dissociation and probe lasers were chosen with consideration of the low azomethane absorption cross section $\left(1.9 \times 10^{-20} \mathrm{~cm}^{2}\right)$ and the relative ease of multiphoton absorption. While multiphoton processes are less important at low power densities, we were unable to operate at such low powers that these effects were eliminated, while still producing practical signal count rates. In particular, azomethane, methyldiazenyl and methyl ions appeared in the mass spectrum as a consequence of absorbing three or more photons from either the dissociation or probe lasers. In order to maximize the desired signal while keeping the non-resonant ions at a workable level, we used a laser spot size of $0.5 \mathrm{~mm}^{2}$ at the crossing with the molecular beam. This relatively large spot kept the power density reasonably low, while increasing the interaction volume and thus the number of molecules in the sample.

For detection of methyl radicals, we used the well-known $2+1$ REMPI scheme through the $3 p^{2} A_{2}^{\prime \prime}$ Rydberg state [117]. The transition between vibrationless methyl states lies at $333.4 \mathrm{~nm}$ (designated $0_{0}^{0}$ ) while that between states with a single quantum of umbrella vibration $\left(v_{2}=1\right)$ lies at $329.4 \mathrm{~nm}$ (designated $\left.2_{1}^{1}\right)$. These states have a high predissociation rate, so individual rotational lines are not resolved. Also, the dissociation rate increases with vibrational state, so that higher vibrational levels are too weak to observe. In detecting methyl radicals using this scheme, we positioned the laser frequency at the maximum of the REMPI peaks, which corresponds. to the narrow cluster of broadened Q-branch lines. Because of the broad laser bandwidth $\left(0.1 \mathrm{~cm}^{-1}\right)$ and the predissociation broadening, most of the rotational states should have been detected. Although a slight detuning of the probe frequency did not affect the shape of the TOFMS profiles, a more detailed study would be needed to fully characterize the degree (if any) of methyl rotational-state selection in our measurements. Indeed it will be desirable in the future to study in detail the rotational 
state dependence of the kinetic energy release, however a different methyl detection scheme will be required. The new frequency-modulated absorption method would be a promising possibility [22], but only if a sufficiently fast infrared detector can be developed.

We chose the core-sampling time-of-flight mass spectrometry technique for measuring the recoil kinetic energy in azomethane dissociation. The principle of this technique has been described in detail by Syage [16] and by Simpson et al. [118]. In this method, only those photofragments with recoil velocity directed (nearly) parallel to the ion flight axis are detected, providing a near one-to-one correspondence between recoil velocity and ion flight time. In principle, the same information can be extracted from a non-core-sampled TOFMS peak or an ion image, but we preferred the coresampling method because it allows immediate qualitative characterization of the raw data. This was particularly important for characterizing multiphoton processes.

The basic principle of the method is depicted in Fig. 5.4. It consists standard Wiley-McClaren extraction optics, a flight tube, and a charged particle detector such as a microchannel plate, with a $2.5 \mathrm{~mm}$ aperture in front to core the photofragment sphere. It is desirable to operate the extraction optics in the delayed-pulse extraction mode, with the 'energy-focussing' condition [71] detuned so that the flight axis dimension of the photofragment sphere is spread out in space (and thus arrival time). In this mode, the first extraction region remains field-free for roughly 500 ns while the ionized photofragment cloud expands to a diameter of several millimeters. When the field is switched on, the initially backward-flying ions are at a higher potential energy, so they pass the forward flying ions during flight and arrive earlier at the detector. The energy resolution in these experiments is limited primarily by the large laser spot size. A core-sampled REMPI-TOFMS profile of methyl radicals (with a single recoil energy) produced by methyl iodide dissociation show a time resolution of 20 ns under typical ion extraction conditions. This is roughly $10 \%$ of the azomethane TOFMS profile widths, which is sufficient resolution for the type of structure that we are interested in observing.

Because of the molecular beam velocity, ions travelling down the flight tube have a small velocity component in the plane perpendicular to the detector flight axis. 


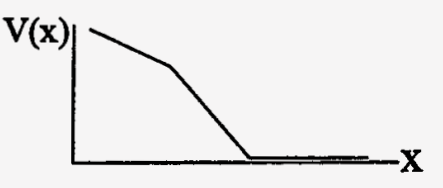

(a)
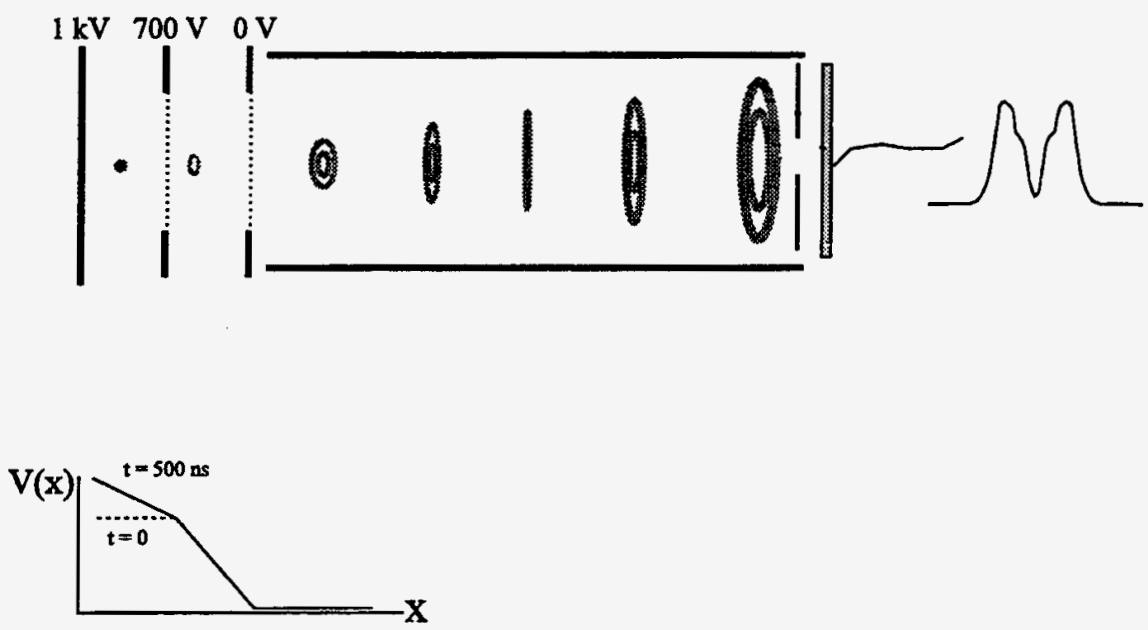

(b)

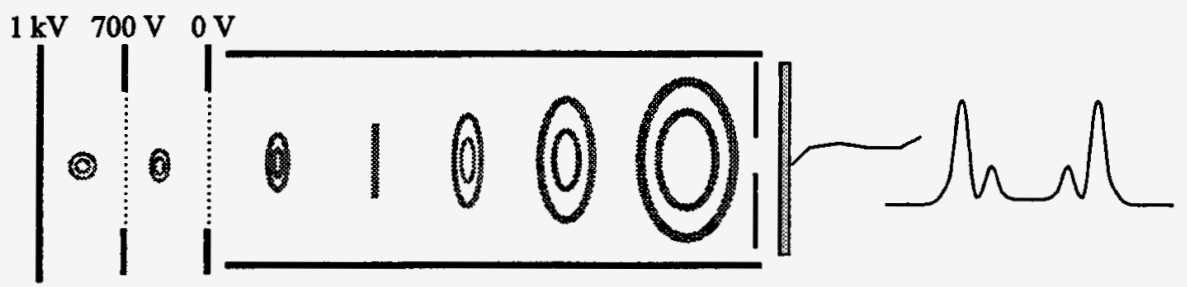

Figure 5.4: Principle of core-sampling TOFMS technique, using delayed-pulse extraction. An aperture in the flight tube extracts a core from the product cloud, discarding large $v_{x}$ and $v_{y}$ components and improving velocity resolution. In standard WileyMcClaren operation (a), extraction fields are constant, and ion cloud may remain compressed at detector. In (b), ion cloud expands while extraction region remains field free. Products are spread over a wider range of potential energy when the field turns on, resulting in an expanded cloud at the detector. 
Under typical extraction conditions for mass 15 , this results in an offset of several millimeters from the detector center. Since it is important that the aperture core the center of the photofragment cloud, we mounted it on an xyz manipulator which could be adjusted from outside of the vacuum chamber. In order to optimize the centering, we maximized the methyl REMPI signal from multiphoton dissociation of methyl iodide, which is peaked strongly near zero kinetic energy, or similarly, the non-resonant multiphoton methyl signal from azomethane dissociative ionization (see Sec. 5.4.1). Despite the broader maximum at zero velocity, it was preferable to use azomethane for this alignment, since the molecular beam velocity was certain to be the same one used for the dissociation experiments.

\subsection{Results and Discussion}

\subsubsection{Multiphoton Processes}

At the laser power densities used in our experiments, both the dissociation and probe lasers produce ions of azomethane, methyl and methyldiazenyl. There are several reasons for seeking a clearer understanding of the processes which produce these ions. First, since the nonresonantly-produced $\mathrm{CH}_{3}^{+}$ions interfere with our desired REMPI ions, we would like to know the origin of these ions and how they may be reduced or eliminated. Second, the presence of the methyldiazenyl ion $\mathrm{CH}_{3} \mathrm{~N}_{2}^{+}$ raises the question of whether it is produced by dissociative ionization or by direct ionization of the neutral methyldiazenyl radical. Finally, the dynamics of azomethane dissociative ionization may itself be of fundamental interest as a further window into three-body dissociations.

Time-of-flight mass spectra provide the first clues to help assign the important multiphoton processes. Mass spectra were recorded for ions produced at both the dissociation and probe wavelengths, under the soft focussing conditions described in the previous section. These data suggest that the methyldiazenyl ion is produced through the dissociative ionization process:

$$
\mathrm{CH}_{3} \mathrm{~N}_{2} \mathrm{CH}_{3} \stackrel{\geq 3 h \nu}{\longrightarrow} \mathrm{CH}_{3}+\mathrm{CH}_{3} \mathrm{~N}_{2}^{+}
$$


As shown in Fig. 5.5, the relative amounts of azomethane and methyldiazenyl ions change considerably with wavelength. Both mass spectra were recorded over a range of pulse energies between $3 \mathrm{~mJ}$ and $12 \mathrm{~mJ}$, with little variation in the azomethane/methyldiazenyl ion ratio at a given wavelength, confirming that the effect is due to the wavelength change rather than laser power density variations. The reduction in parent ion at shorter wavelength could result from a different weighting of two separate dissociation channels (i.e. ionization vs. dissociative ionization) or from a reduction in the dissociation lifetime of an activated parent ion. Mass spectra recorded under faster extraction conditions favor the dissociation lifetime explanation. The change in width of the methyldiazenyl peak is also consistent with a dissociation mechanism, where the extra energy available at the shorter wavelength imparts greater recoil energy to the fragments. Furthermore, if the methyldiazenyl ions resulted from ionization of the neutral radical, we also would not expect a change in their kinetic energy with wavelength.

The energy level diagram in Fig. 5.6 clarifies which processes may contribute to the mass spectrum. The ionization potential of azomethane is around $9 \mathrm{eV} \mathrm{[109],}$ so three photons of either UV wavelength should be sufficient to ionize it. Without knowing the methyldiazenyl ionization potential, we cannot calculate the energy for Process 5.3. However, from the mass spectra, we can conclude that this energy must lie below that of three $355 \mathrm{~nm}$ photons, and may even lie below the azomethane ionization potential. The energy of the process

$$
\mathrm{CH}_{3} \mathrm{~N}_{2} \mathrm{CH}_{3} \stackrel{\geqq 3 h \nu}{\longrightarrow} \mathrm{CH}_{3}^{+}+\mathrm{CH}_{3}+\mathrm{N}_{2}
$$

can be calculated relative to azomethane by adding the dissociation energy of azomethane $(\sim 22 \mathrm{kcal} / \mathrm{mol}$ or $0.95 \mathrm{eV})$ to the ionization potential of the methyl radical $(9.85 \mathrm{eV})$, however the former is not known to within an accuracy better than $0.2 \mathrm{eV}$. Given this uncertainty, it is possible that Process 5.4 lies at a higher energy than three photons of either $330 \mathrm{~nm}$ or $355 \mathrm{~nm}$ and thus requires a fourth photon to produce methyl radicals. This would be consistent with the much smaller quantity of methyl ions in both mass spectra of Fig. 5.5. While Process 5.4 could be 3-photon-accessible but simply occur with low probability, this explanation would be more convincing if 


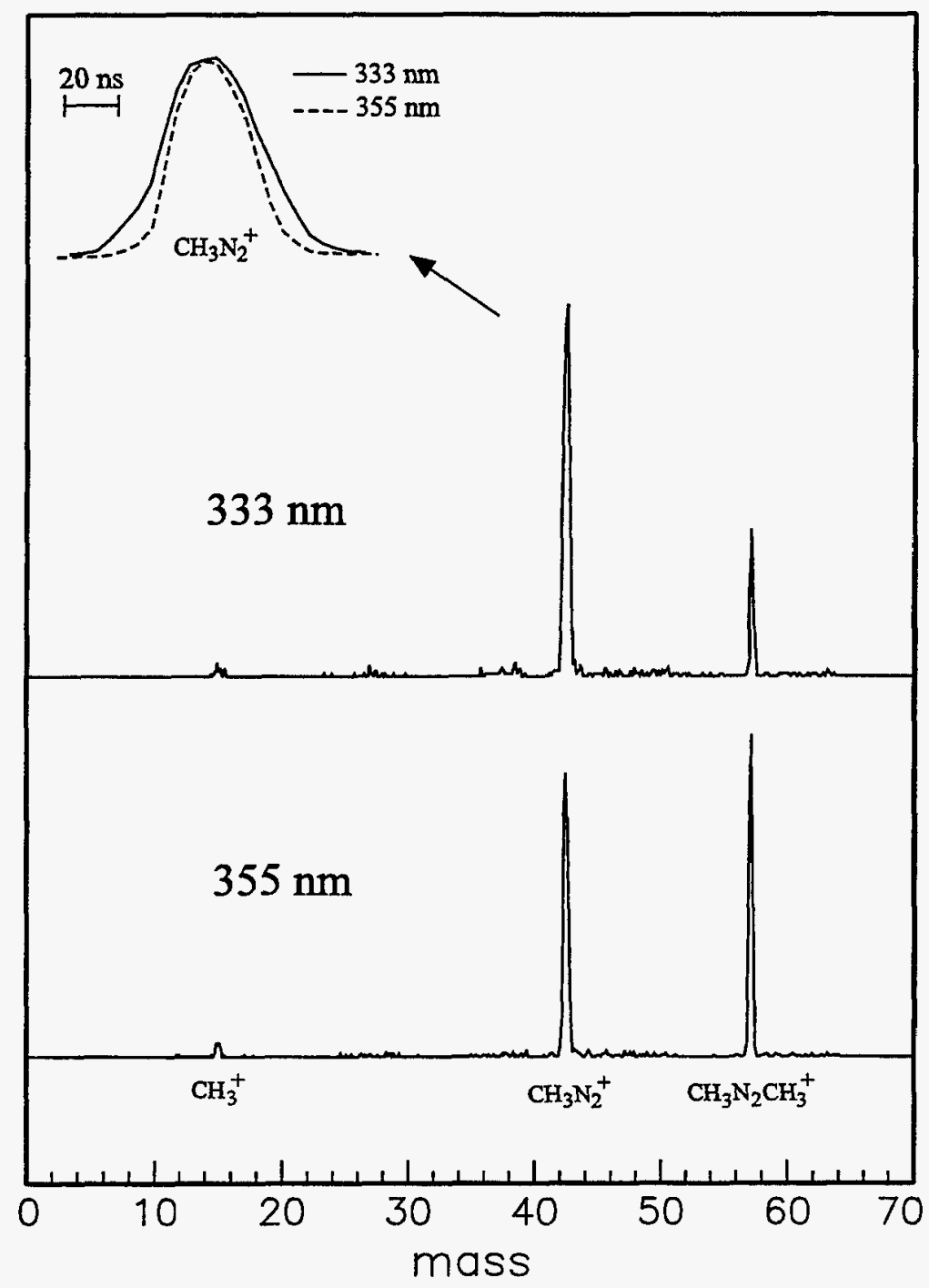

Figure 5.5: Comparison of mass spectra at $330 \mathrm{~nm}$ and $355 \mathrm{~nm}$ : dissociation of the azomethane ion is more efficient at shorter wavelength. The methyldiazenyl ion peak widths (inset) show that kinetic energy release is greater at $330 \mathrm{~nm}$. 
there were a larger difference in the relative quantity of methyl ions produced at the two wavelengths.

In order to confirm these assignments, we measured ion yield as a function of energy for single-photon absorption of vacuum ultraviolet light around the azomethane ionization potential. These measurements were carried out at Endstation 9.01 of the Advanced Light Source at Lawrence Berkeley National Laboratory. A molecular beam of azomethane was directed through a pinhole aperture and then crossed with a VUV beam from the synchrotron before entering a quadrupole mass spectrometer. Following mass selection, the ions were detected with a Daly-type ion counter. Fig. 5.7 shows the results of this measurement. Methyldiazenyl ions begin to appear around $9 \mathrm{eV}$, although because of the large bandwidth of the ALS light $(\sim 0.5 \mathrm{eV})$, the true onset is probably somewhat higher. Based on this measurement, dissociative ionization remains a good candidate to explain the methyldiazenyl ions, although ion pair formation from a potential energy surface for the azomethane neutral cannot be ruled out. Methyl ions appear around $11.5 \mathrm{eV}$, consistent with the scarcity of these ions following multiphoton excitation at both UV wavelengths. This onset is also close to the predicted ionization potential to form ${ }^{2} A_{u}$ azomethane ion [109], which may then correlate to methyl ion products. We observed only a negligible amount of azomethane parent ion following VUV absorption. This does not contradict the UV mass spectra, however, since the parent ions have much more time to dissociate during the $45 \mu \mathrm{s}$ flight time to the detector, than during the $500 \mathrm{~ns}$ field-free delay period of the TOFMS technique. (In the latter technique, parent ions which dissociate during extraction will be smeared over a range of flight times.)

\subsubsection{Ultraviolet Dissociation}

\section{Methyl appearance times and TOFMS profiles}

The major dynamical questions addressed in this work are the timing of the primary and secondary methyl loss and the partitioning of energy between translation and umbrella vibration. As discussed in the introduction, there are conflicting reports in the literature on both of these issues. We have measured product appearance times 


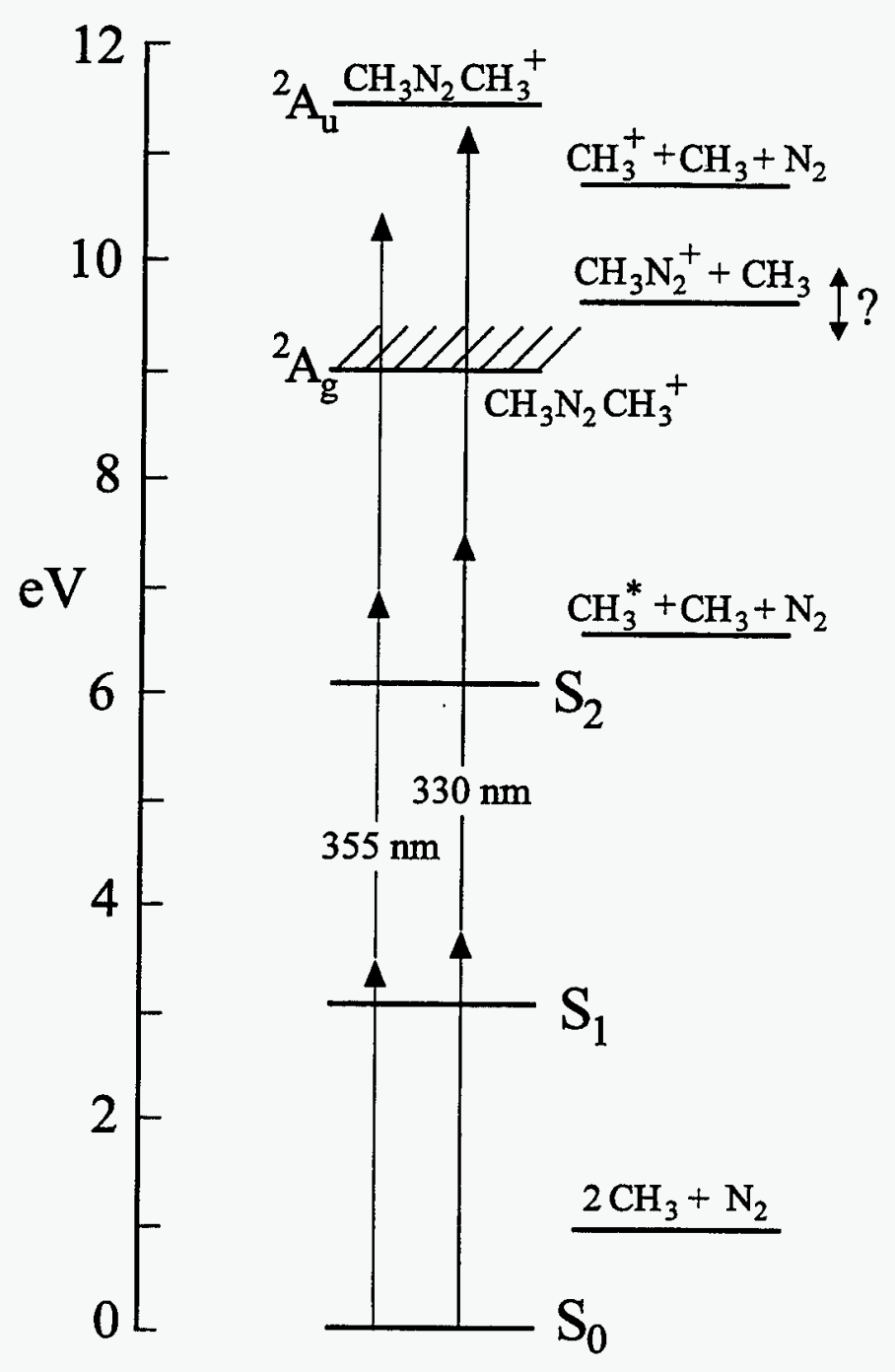

Figure 5.6: Energy level diagram showing relevant ionization and dissociation channels. The energies for $\mathrm{CH}_{3} \mathrm{~N}_{2} \mathrm{CH}_{3}^{+}\left({ }^{2} \mathrm{~A}_{u}\right)$ and $\mathrm{CH}_{3}+\mathrm{N}_{2}+\mathrm{CH}_{3}^{+}$come from ab initio calculations, while that for $\mathrm{CH}_{3} \mathrm{~N}_{2}^{+}+\mathrm{CH}_{3}$ is unknown. 


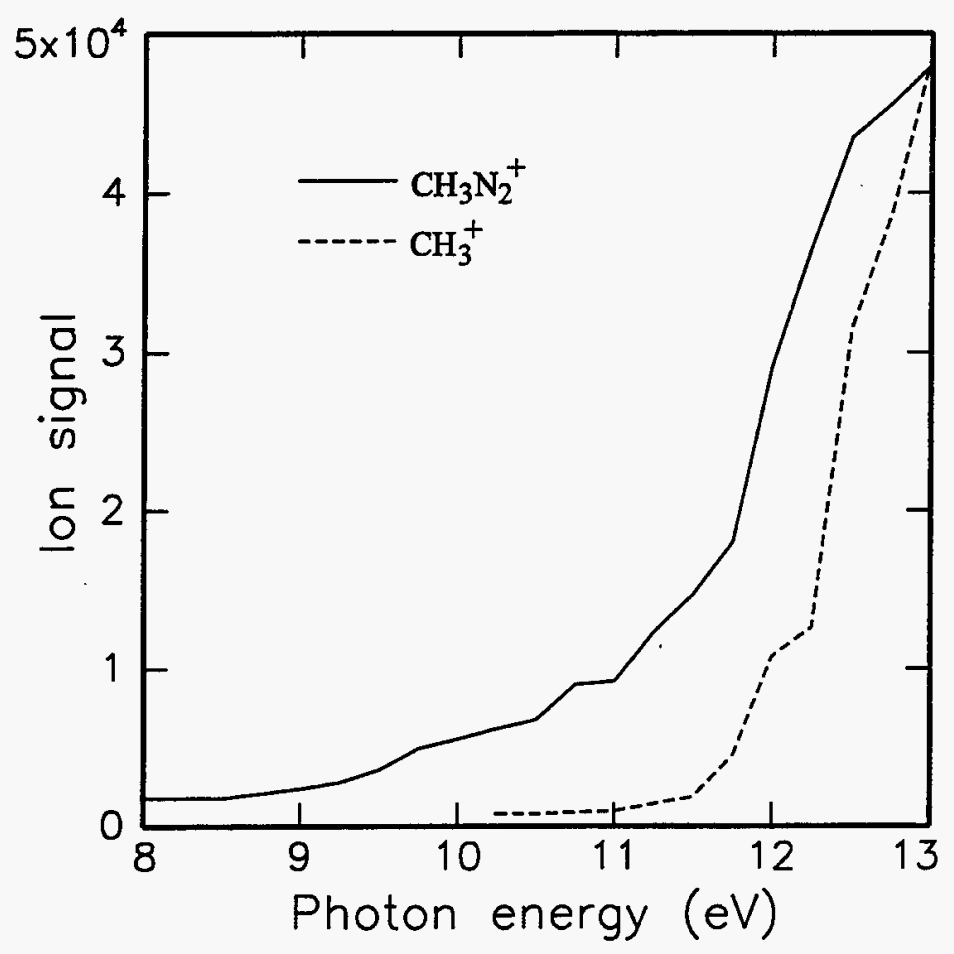

Figure 5.7: Ion yield from VUV dissociative ionization of azomethane. Apparent onsets are broadened by $0.5 \mathrm{eV}$ bandwidth of the synchrotron light source. Negligible azomethane ion yield suggests low cross section and/or high dissociation probability near threshold

and core-sampled TOFMS profiles to help provide a clearer picture.

In a straightforward experiment, we have carried out the analogue of Weisman's two-laser time delay measurement, now using REMPI rather than CARS as the probe method. Total methyl signal was collected (no core) and averaged with a boxcar integrator at 11 different time delays between the dissociation laser and the REMPI probe laser. The results of this measurement are shown in Fig. 5.8 for the $0_{0}^{0}$ and $2_{1}^{1}$ REMPI transitions. Both data sets are fit reasonably well with a rise time corresponding to the integrated convolution of two Gaussian profiles, which approximate the laser time profile. Our result is clearly different from Weisman's result [113], which showed a risetime nearly twice as long for the vibrationless methyls. Without knowing further details of their method, we can not easily propose an alternative explanation for their result. However, interpretation of their gas cell measurement (which involved partial collisional relaxation of the products) is likely to be more 
complex than for the collisionless conditions in a molecular beam experiment. If our measurement is correct, then there no longer exists a conflict in assignment of the dissociation mechanism with regard to the timing of bond breakage. While our measurement cannot eliminate the possibility of a sequential mechanism, the results of North et al. point strongly toward concerted bond breakage. A concerted dissociation is fully consistent with several recent $a b$ initio calculations (including one by Andrews and Weisman [119]) which predict a barrier to methyldiazenyl dissociation of less than $2 \mathrm{kcal} / \mathrm{mol}$.

In studying energy partitioning here, we are interested primarily in the correlation between two types of motion. For example, we would like to know whether the fast-moving methyl photofragments are vibrating more or less than those which recoil slowly. Such correlations can provide detailed information about the shape of the potential energy surface. The goal of the experiments described in the remainder of this section is to obtain translational energy distributions for methyl fragments with zero and one quantum of umbrella-mode vibrational energy. We measured coresampled REMPI-TOFMS profiles for dissociation at $355 \mathrm{~nm}$ and at $330 \mathrm{~nm}$. The measurements at the former wavelength allow a more direct comparison with the results of North et al. and Weisman et al. The single-laser experiment at the latter wavelength allows comparison with the results of Fairbrother et al. Furthermore, in the two-laser experiment, a significant fraction (20-30\%) of the signal results from dissociation by the probe laser, and one would like to be able to subtract out this contribution if necessary.

Since a significant number of methyl ions are produced by non-resonant processes, it is necessary to subtract this background signal from the desired dissociation signal. Every time a TOFMS profile was collected with the REMPI laser tuned to a methyl resonance, we subsequently collected a background profile with the REMPI laser tuned between the $0_{0}^{0}$ and 21 REMPI transitions, so that only the non-resonant ions were produced, while all other experimental conditions were kept the same. Typical signal, background, and difference profiles for both REMPI transitions are shown in Fig. 5.9.

The background-corrected TOFMS profiles appear in Figs 5.10 and 5.11 


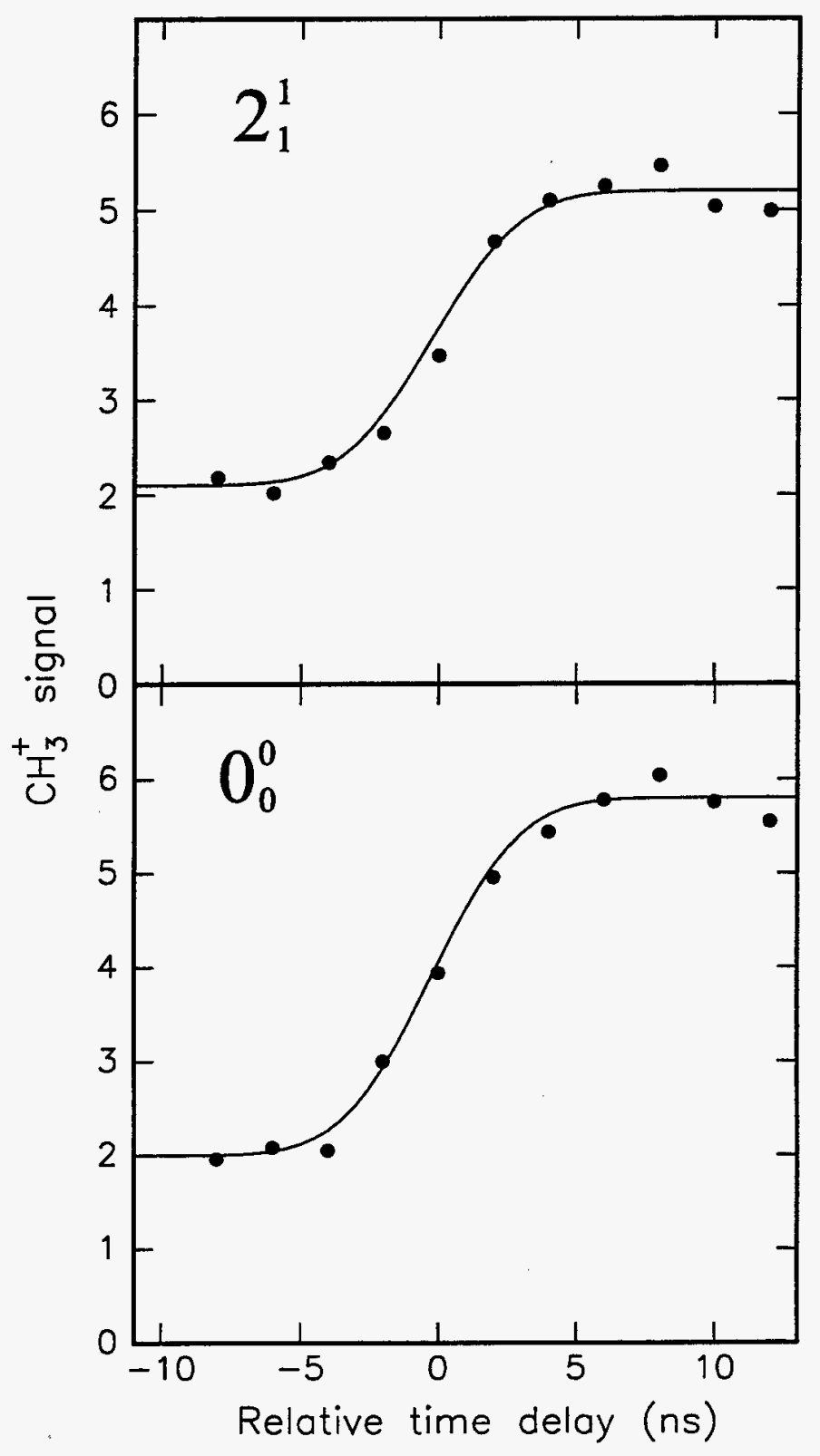

Figure 5.8: Dependence of $\mathrm{CH}_{3}$ REMPI signal on laser time delay. Equivalent appearance times for methyl radicals in both vibrational states are limited by the laser pulsewidth. The REMPI signal is offset from zero due to dissociation by the REMPI laser and to a lesser extent $(<20 \%)$ from nonresonant multiphoton processes (See Section 5.4.1). 


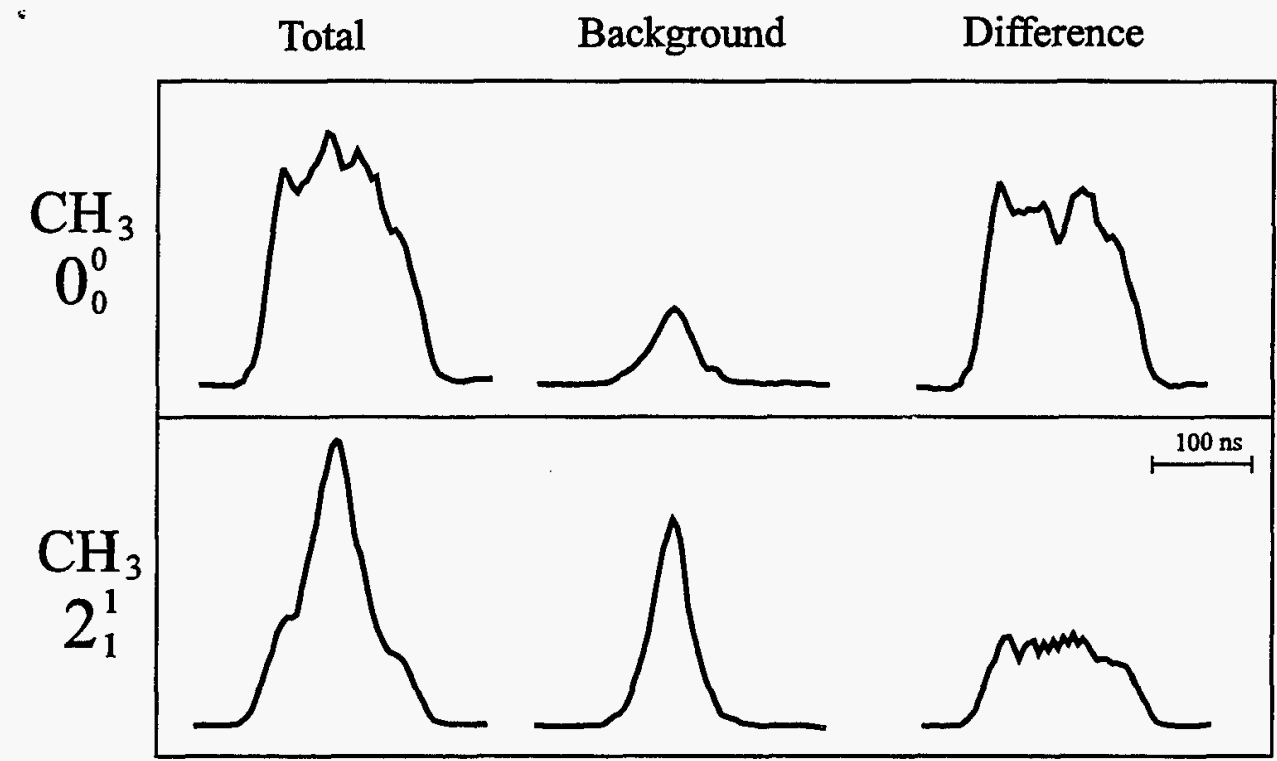

Figure 5.9: Subtraction of non-resonant signal from REMPI-TOFMS signal profiles. Non-resonant profiles were obtained with the probe laser tuned between the $0_{0}^{0}$ and $2_{1}^{1}$ REMPI transitions. The background is relatively much larger for the weaker $2_{1}^{1}$ transition.

for $355 \mathrm{~nm}$ and $330 \mathrm{~nm}$, respectively. In practice, background subtraction was not straightforward. Because of slow drifts in experimental conditions, backgroundcorrected profile data which were collected several hours apart were not reproducible to our satisfaction. The situation improved somewhat by alternating data collection on- and off- resonance every $10 \mathrm{~K}-20 \mathrm{~K}$ laser shots, however, some drifts even occurred on this shorter time scale. Any faster alternation was impractical and probably subject to a new set of faster experimental drifts. In the end, we chose to normalize the intensity of a given profile based on the integrated ion signal for all ion masses other than 15 (methyl ion). Performing the background subtraction on these normalized profiles provided the most reproducible difference profiles. The error bars in the TOFMS profiles of Figs 5.10 and 5.11 are standard deviations associated with summing multiple data sets. Although still large, they are superior to those obtained with only a raw background subtraction. The sources of variation between measurements may be due to such effects as changes in the laser spatial profile (and thus local power density) or slight laser beam walk during wavelength changes. These effects 


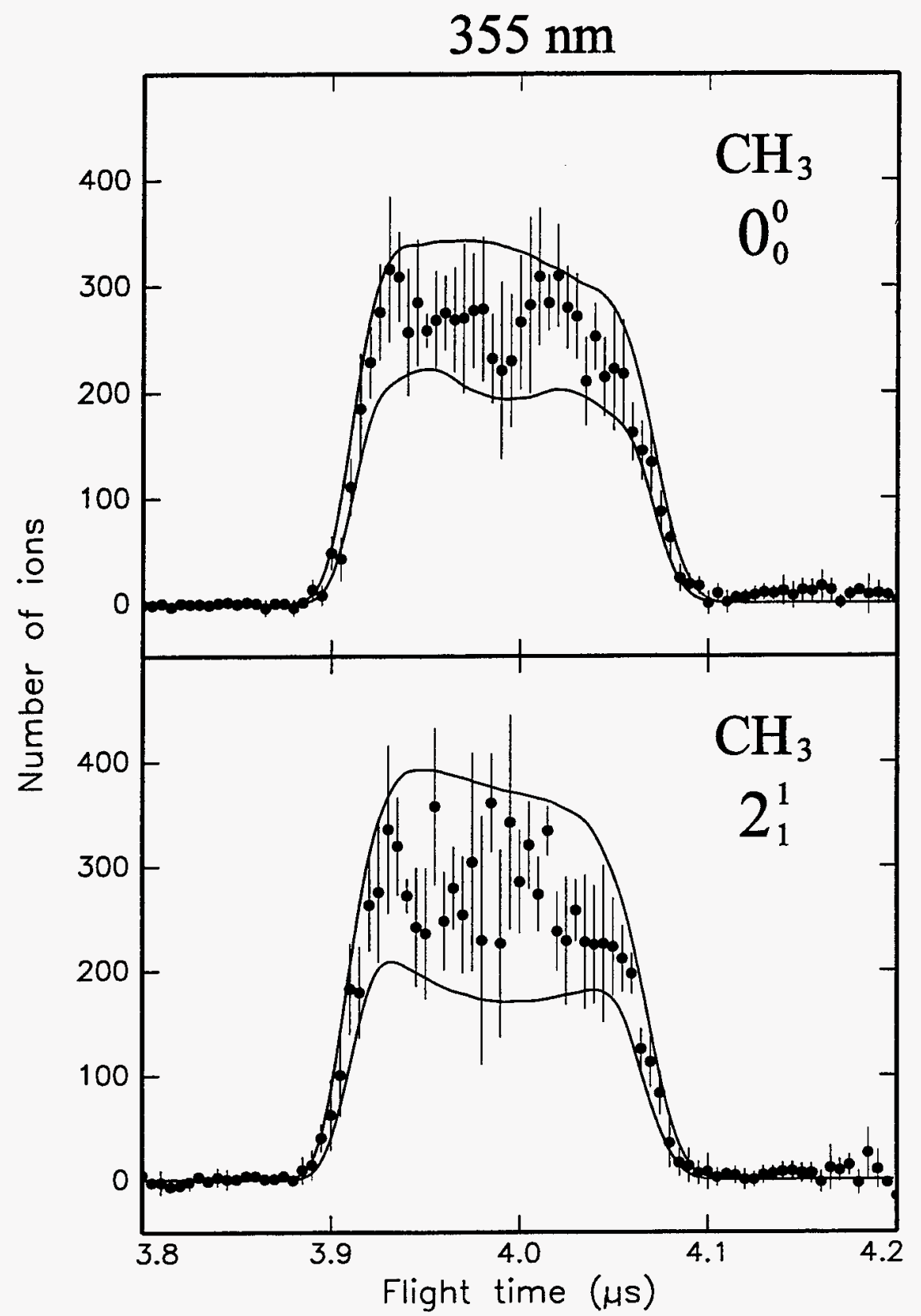

Figure 5.10: Methyl REMPI-TOFMS profiles for $355 \mathrm{~nm}$ dissociation of azomethane. Error bars are standard deviations associated with summation of different data sets. Upper and lower limiting fits were obtained by forward convolution of translational energy distributions in Fig. 5.14. 


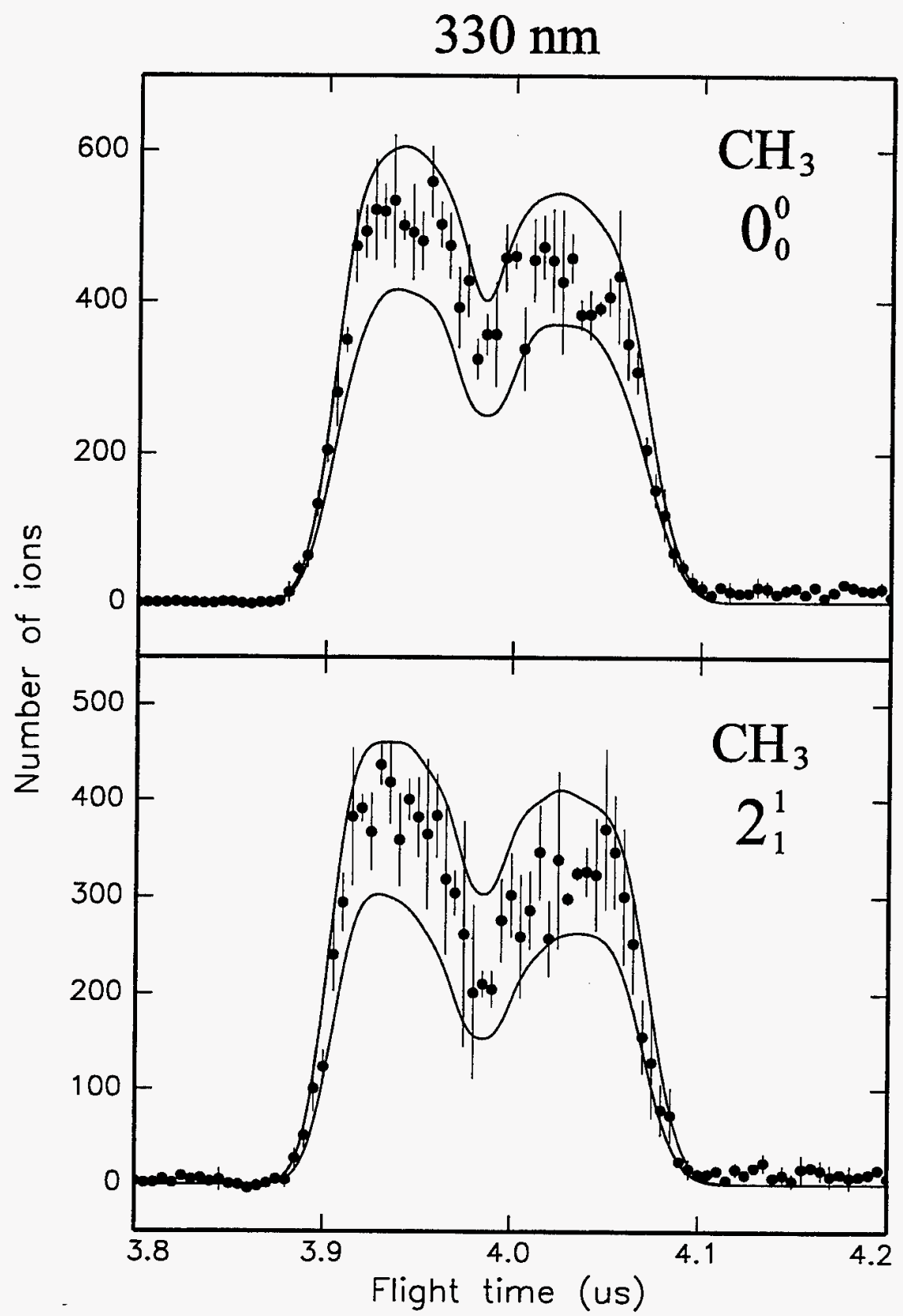

Figure 5.11: Methyl REMPI-TOFMS profiles for $330 \mathrm{~nm}$ dissociation of azomethane. A single laser pulse was used for dissociation and probe. Error bars are standard deviations associated with summation of different data sets. Upper and lower limiting fits were obtained by forward convolution of translational energy distributions in Fig. 5.15. 
may distort ion signals in either a positive or negative manner, which is consistent with the observation that the normalization factors for the off-resonance mass spectra could be both greater or less than those of the on-resonance spectra.

The scatter in the points reflects primarily the shot statistics and is consequently less for the $0_{0}^{0}$ state, which had a background signal much less than the total signal. The scatter for the $2_{1}^{1}$ state is especially large near the peak center, due to the large background signal at those flight times. Especially in the $0_{0}^{0}$ profiles, there appears to be structure which is more pronounced than the scatter. In fitting the profiles, however, we chose to roughly bracket the error bars near their maxima and minima with two nearly-structureless fits. There are two reasons for this. First, the error bars are considerably wider than the point scatter and comparable in size to the apparent structure in the profiles. Second, the structure is asymmetric in a way which cannot be explained by the well-understood distortion of the product cloud during extraction. Since it is possible that the structure is real but distorted due to shortcomings of the background subtraction, we have also attempted to fit this structure. This fit is show in Fig. 5.16 for comparison with the bimodal translational energy distribution of North et al. [115]

\section{Obtaining Translational Energy Distributions}

The data fitting method involves forward convolution of a manually-adjusted translational energy distribution, $P(E) \equiv d N / d E$, where $N$ is a number of molecules. For purposes of fitting, a continuous translational energy distribution is treated as a discrete array of closely spaced delta functions weighted by the envelope of the distribution. The derivative chain rule converts $P(E)$ to a speed distribution: $P(v)=$ $P(E) d E / d V \propto P(E) \sqrt{E}$. A single delta function in energy corresponds to a spherical shell in $\mathbf{v}$-space, which is projected onto the $z$-axis (detector axis) in deriving a TOFMS profile. A spherical shell in velocity space yields a box-shaped profile in $v_{z}$ space, as shown in Fig. 5.12. Applying Newton's second law for the extraction optics and rearranging the kinematic equations yields an expression for flight time 
a)
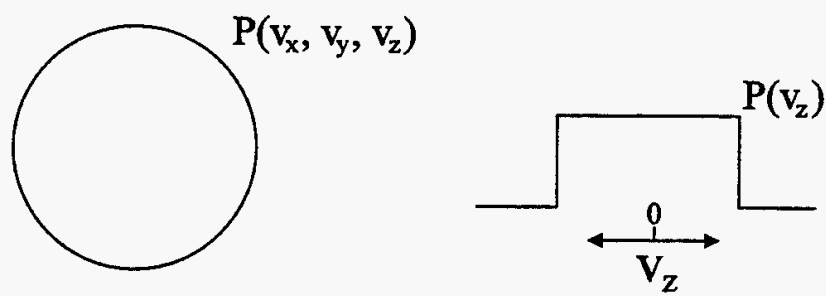

b)
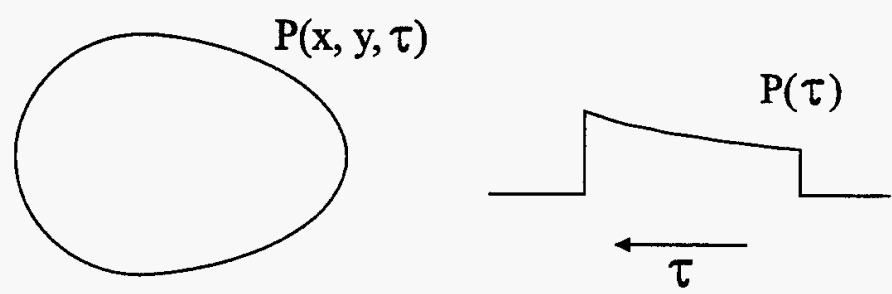

c)
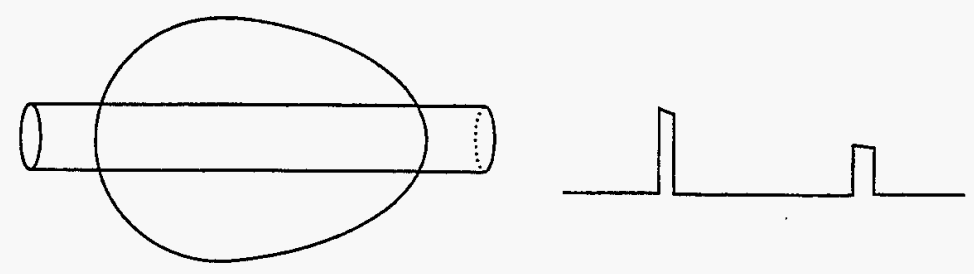

d)
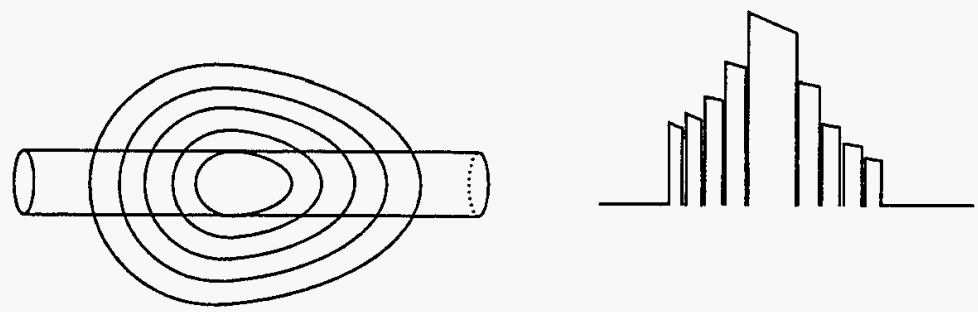

Figure 5.12: Simulating core-sampled TOFMS profiles. (a) Spherical shell in velocity space produces box shape in $v_{z}$ space. (b) Transform to time-of-flight and multiply $P\left(v_{z}\right)$ by $d v_{z} / d \tau$. (c) remove all portions of profile where $v_{x}^{2}+v_{y}^{2}$ is greater than core radius. (d) Sum profiles for all energies, appropriately weighted according to $P(E)$. 
$\tau=t_{1}+t_{2}+t_{3}$ as a function of $v_{z}$ :

$$
\begin{aligned}
t_{1} & =t_{d e l}+\left(2 l_{1} \chi_{1}+v_{z}^{2} \chi_{1}^{2}\right)^{1 / 2}-v_{z} \chi_{1} \\
t_{2} & =\left[2 L_{2} \chi_{2}+\left(v_{z}^{2}+\frac{2 q}{m} V_{1}\right) \chi_{2}^{2}\right]^{1 / 2}-\left(v_{z}^{2}+\frac{2 q}{m} V_{1}\right)^{1 / 2} \chi_{2} \\
t_{3} & =L_{3}\left[v_{z}^{2}+\frac{2 q}{m}\left(V_{1}+V_{2}\right)\right]^{-1 / 2}
\end{aligned}
$$

with the following definitions. (The dimensions and voltages are also indicated in Fig. 5.13.):

$t_{d e l}$ - time delay between laser fire and repeller voltage on

$L_{1}-$ distance from interaction region to first mesh

$l_{1}-$ distance of particle from first mesh after time delay $l_{1}=L_{1}-v_{z} t_{d e l}$

$L_{\text {rep }}$ - length of first extraction stage (repeller to mesh)

$L_{2}, L_{3}$ - length of second extraction stage and flight tube, resp.

$V_{1}-$ voltage at $l_{1}: V_{1}=\left(V_{\text {rep }}-V_{2}\right) l_{1} / L_{r e p}$

$V_{\text {rep }}, V_{2}-$ voltages at repeller and mesh, resp.

$$
\begin{aligned}
& \chi_{1}-\frac{m}{q} L_{\text {rep }} /\left(V_{\text {rep }}-V_{2}\right) \\
& \chi_{2}-\frac{m}{q} L_{2} / V_{2}
\end{aligned}
$$

In practice, it is difficult to measure all of the above quantities precisely, particular the repeller pulse voltage, the time delay between the laser firing and the extraction pulse, and the position of the laser in the interaction region. For this reason, some sort of indirect calibration is necessary to determine the relationship between velocity and flighttime. The most reliable way is to dissociate a molecule with a known, sharp kinetic energy release. We used methyl iodide for this purpose. Since our REMPI scheme selects a narrow range of internal states and the atomic iodine energy levels are spaced far apart, we can easily resolve peaks which correspond to a (nearly) single kinetic energy. By repeating this calibration over a range of extraction voltages and time delays, one could in principle solve a set of simultaneous equations to determine the true values of the various experimental parameters. This is useful if the parameters are expected to change between measurements. In practice, it is easier to simply repeat a single 'relative' calibration when conditions change. 


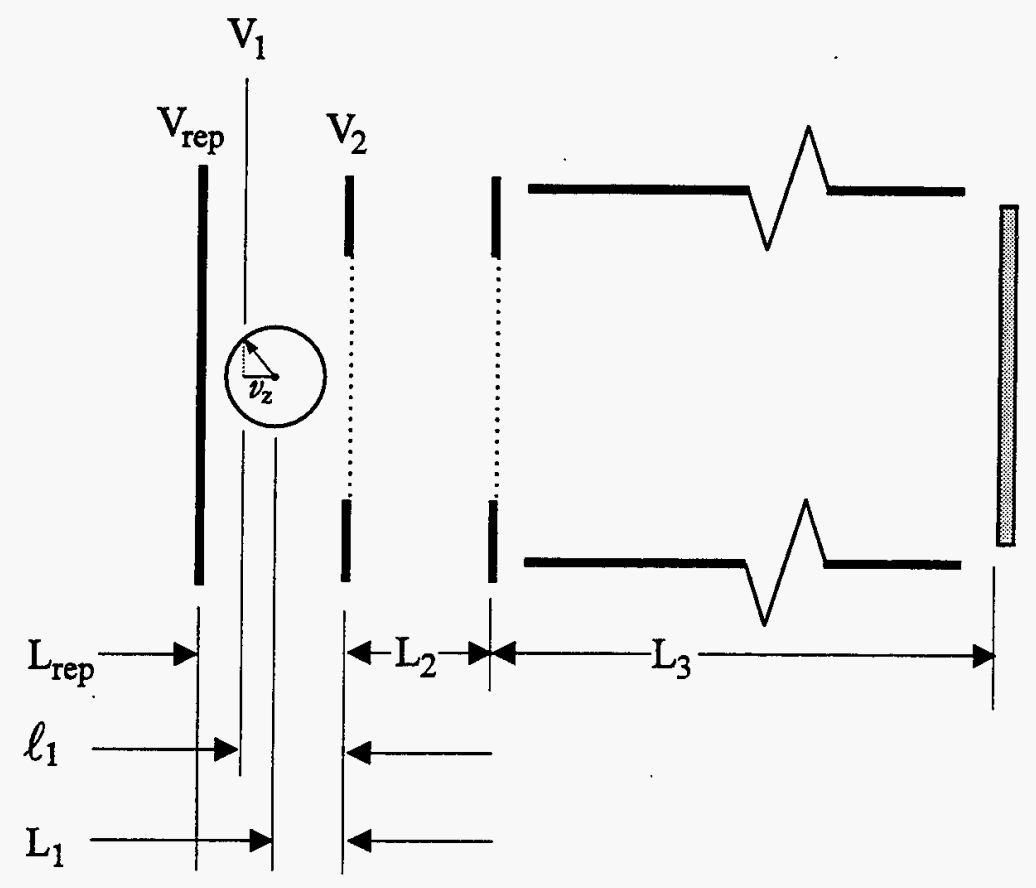

Figure 5.13: Notation for voltages and dimensions of mass spectrometer.

Because of the extraction fields, the sphere in velocity space is distorted in position space (along $z$ ) and likewise in flight time [Fig. 5.12(b)], so the box shape distribution $P\left(v_{z}\right)$ should be multiplied by $d v_{z} / d \tau=\left(d t_{1} / d v_{z}+d t_{2} / d v_{z}+d t_{3} / d v_{z}\right)^{-1}$. The necessary derivatives are:

$$
\begin{aligned}
d t_{1} / d v_{z}= & \left(2 l_{1} \chi_{1}+v_{z}^{2} \chi_{1}^{2}\right)^{1 / 2}\left(t_{d e l} \chi_{1}+v_{z} \chi_{1}^{2}\right)-\chi_{1} \\
d t_{2} / d v_{z}= & {\left[2 L_{2} \chi_{2}+\left(v_{z}^{2}+\frac{2 q}{m} V_{1}\right) \chi_{2}^{2}\right]^{-1 / 2}\left(v_{z}+\frac{q}{m} \dot{V}_{1}\right) \chi_{2}^{2} } \\
& -\chi_{2}\left(v_{z}^{2}+\frac{2 q}{m} V_{1}\right)^{-1 / 2}\left(v_{z}+\frac{q}{m} \dot{V}_{1}\right) \\
d t_{3} / d v_{z}= & -L_{3}\left[v_{z}^{2}+\frac{2 q}{m}\left(V_{1}+V_{2}\right)\right]^{-3 / 2}\left(v_{z}+\frac{q}{m} \dot{V}_{1}\right),
\end{aligned}
$$

where $\dot{V}_{1}$ is a derivative with respect to $v_{z}$. Core sampling skims off all points in $P(\mathrm{v})$ where $v_{x}^{2}+v_{y}^{2}$ is greater than the core radius. This results in removal of the central section of $P(\tau)$, as shown in Fig. 5.12(c). Finally, profiles for other energies are summed, with appropriate weightings, and the resulting profile is convoluted with a 20 ns Gaussian, which approximates the experimental time-of-flight resolution.

The translational energy distributions in Figs 5.14 and 5.15 were obtained by fitting the TOFMS profiles. Each distribution is depicted as a range, with limits 
corresponding to the fits through both extremes of the error bars on the TOFMS profiles. A distribution of any shape which fits within the shaded region would be consistent with the measured data. Naturally, these distributions are not normalized.

\section{Discussion}

At least within their uncertainty ranges, the shapes of the translational energy distributions do not show any strong dependence on methyl quantum state. The $330 \mathrm{~nm}$ distributions are qualitatively similar to those for $355 \mathrm{~nm}$, except that they are shifted to slightly higher energy. The falling edges appear to be shifted by $\sim 4 \mathrm{kcal} / \mathrm{mol}$, which is a surprisingly large fraction of the additional available energy of $6 \mathrm{kcal} / \mathrm{mol}$. This type of behavior is generally not consistent with a system in which energy is randomized throughout the molecule prior to dissociation, as azomethane is believed to be. Instead one expects most of the additional energy to remain in internal modes, leaving little extra above the barrier to dissociation and available for translation. For example, assuming a purely statistical 'prior' distribution and a dissociation energy of $50 \mathrm{kcal} / \mathrm{mol}$ (loss of a single methyl), a calculation shows that only $12 \%$ of the extra energy should end up in translation. Therefore, if this effect is real, it may demand a reevaluation of the current mechanistic assignment. However, there are also two possible sources of experimental error which may account for this difference. The first is a slight miscalibration of the energy-to-flighttime conversion, due to unexpected changes in the extraction conditions or laser alignment. The second possibility is a fitting artifact in the wings of the TOFMS profile, where a small change in fit intensity (e.g. caused by an attempt to fit the noise) corresponds to much more dramatic changes in the translational energy distribution.

Our translational energy distributions should be compared to the results of North et al. [115] and Fairbrother et al. [116]. North's distribution shows two distinct features, peaking at $14 \mathrm{kcal} / \mathrm{mol}$ and $5 \mathrm{kcal} / \mathrm{mol}$. It is reproduced in Fig. 5.16(b), superimposed on our $355 \mathrm{~nm}$ distribution for the $0_{0}^{0}$ REMPI transition. We have used a bimodal distribution (dotted line) as an attempt to fit the apparent structure in the $0_{0}^{0}$ TOFMS profile [Fig. 5.16(a)]. The energies of the two components are very similar 


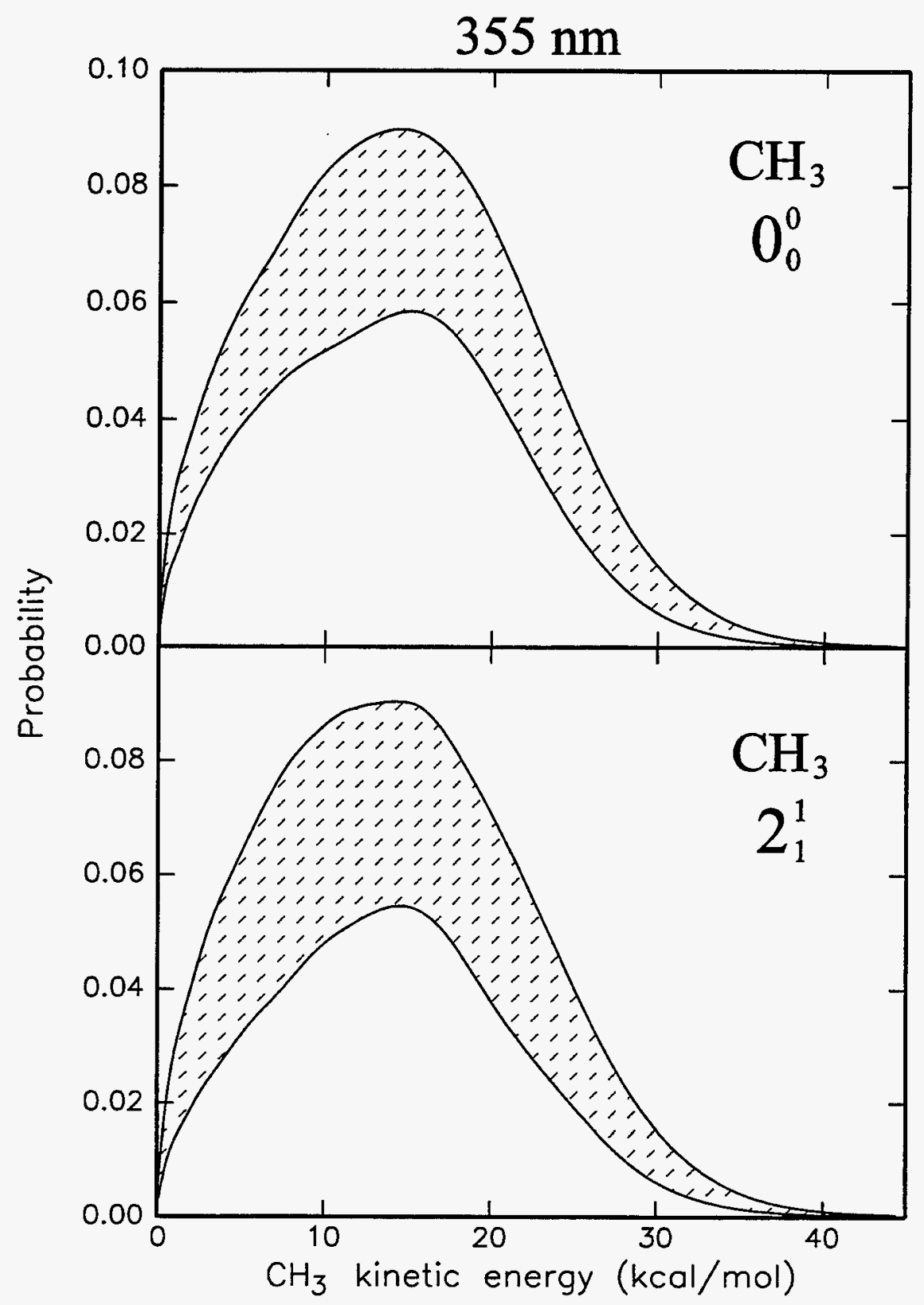

Figure 5.14: Translational energy distributions for $355 \mathrm{~nm}$ dissociation of azomethane. Limits of shaded region correspond to upper and lower fits of Fig. 5.10. 


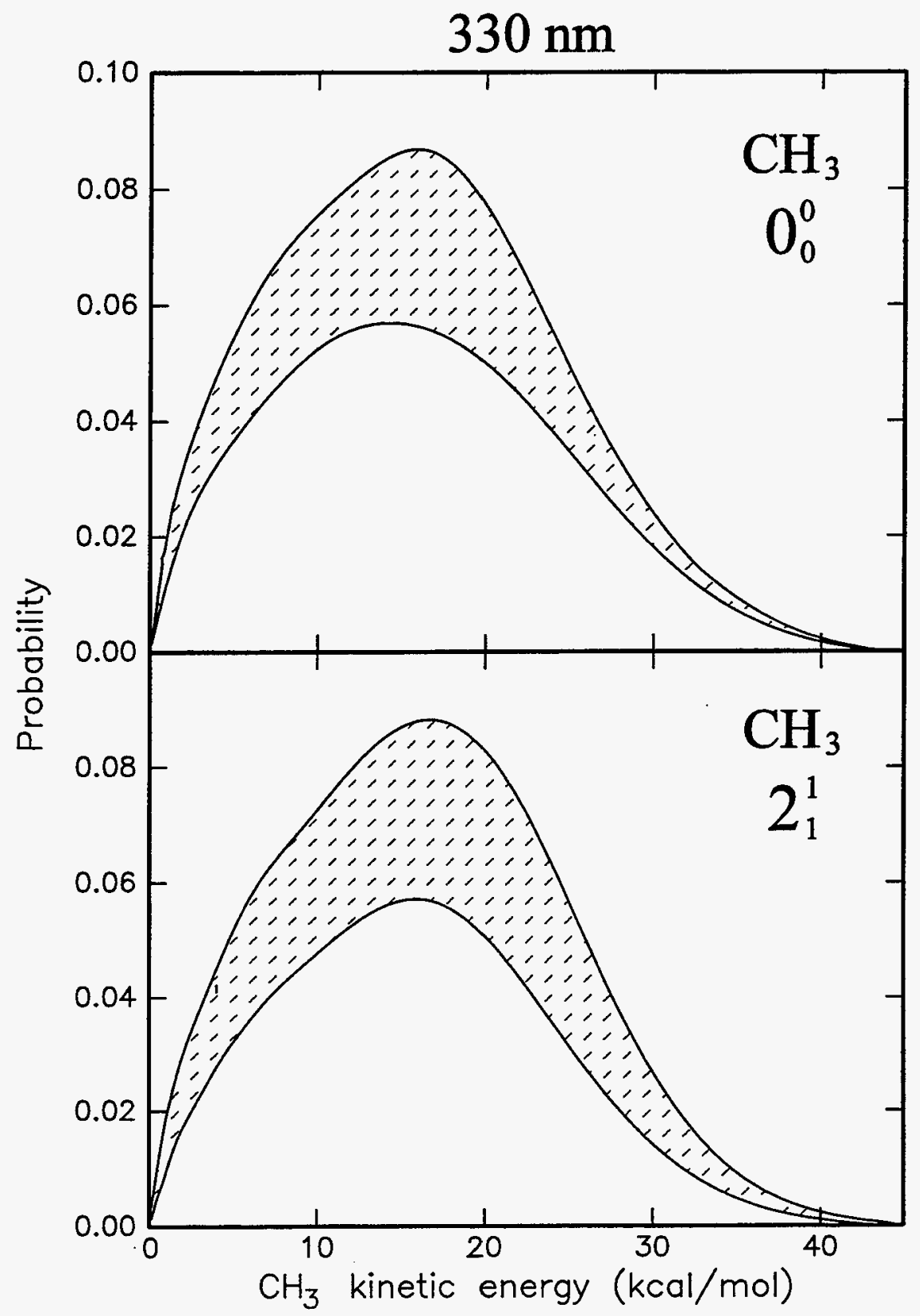

Figure 5.15: Translational energy distributions for $330 \mathrm{~nm}$ dissociation of azomethane. Limits of shaded region correspond to upper and lower fits of Fig. 5.11. 

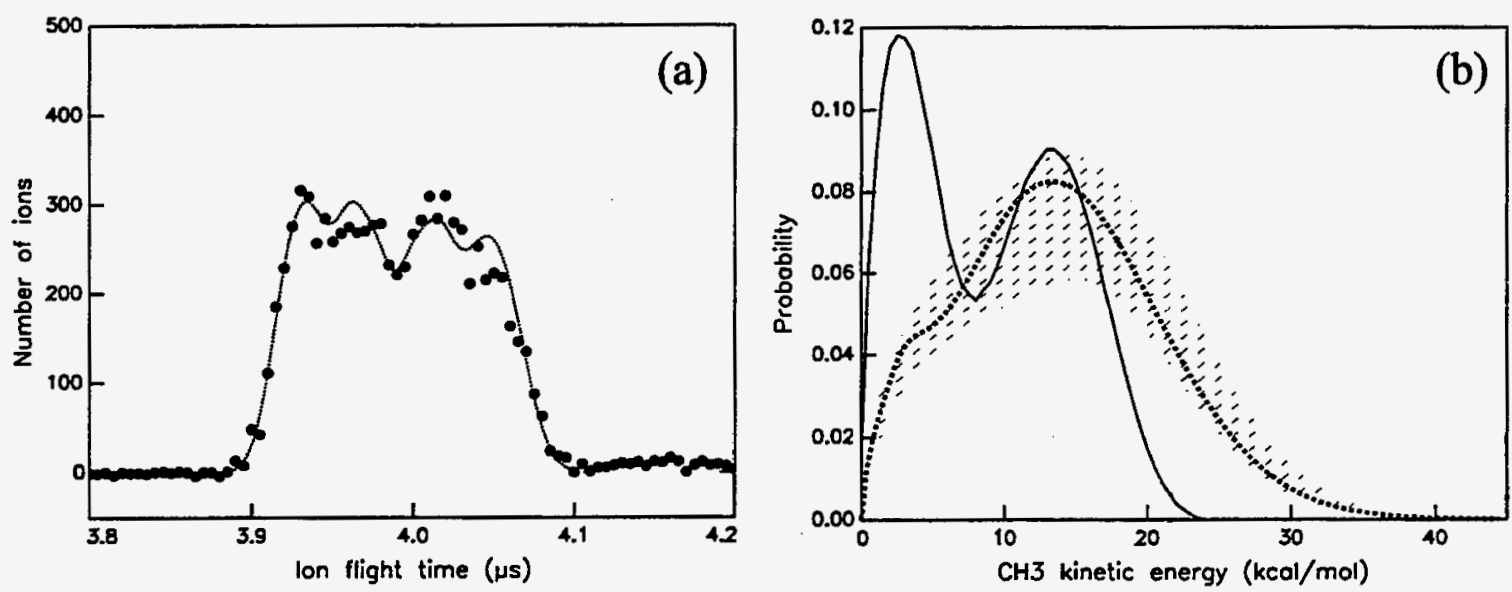

-Figure 5.16: Comparison of translational energy distribution of North et al. [115] with ours. (a) Fit to our data for $355 \mathrm{~nm}$ dissociation ( $0_{0}^{0}$ band) assuming that the structure (4 peaks) is real. (b) Corresponding translational energy distribution (dotted) superimposed on the distribution of North. Shaded region corresponds to our more conservative fit (same as the top of Fig. 5.14).

to those of North et al., although the relative weightings are much different and the components are broader. Our distributions also extend to higher energy. This has been confirmed by ion imaging measurements, where the conversion to energy is quite straightforward, thus the discrepancy cannot be attributed to a calibration error in fitting the profiles. It is possible that the high energy tail of North's distribution is unrealistically low, since this part is very sensitive to their fit at short flight times. North's distribution also does not fit within the uncertainty range of our more conservative fit. At least in the lower energy region, these differences do not necessarily suggest that the measurements disagree, since we have measured only a subset of the methyl quantum states, and dynamical factors may explain the discrepancies. Indeed the purpose of this work is to investigate such differences.

Our results agree qualitatively with those of Fairbrother et al., although our quantitative conclusions are less extreme. They reported an exclusive preference for North's high energy component in their measurements of total TOFMS profiles (i.e. not core-sampled) for the same methyl quantum states. In their analysis, they did not attempt to fit their TOFMS profiles, but were able to extract an average translational energy of $15.8-17.4 \mathrm{kcal} / \mathrm{mol}$. Average energies for our translational 
energy distributions are comparable. A thorough appraisal of their conclusions is not possible without knowing more details of their unspecified analysis, but their conclusion of high selectivity seems overly strong and does not agree quantitatively with our observations.

The indirect conclusions reached by Weisman et al. on energy partitioning in the umbrella mode are much different than our direct observations. They proposed that the first methyl departs impulsively, while the second is lost in a statistical manner. Yet they assign vibrationless methyls to the second step, directly contradicting our observation that the fast methyls dominate. Although these are serious discrepancies, they are not surprising given the likelihood that their time-delay measurements were in error.

Two coarse features of our results require discussion-the non-dependence on vibrational state and the favoring of faster energies in comparison to the results of North et al. At this stage we provide qualitative arguments, but in the future, classical trajectory simulations on the new $a b$ initio potential energy surfaces [109] would be extremely valuable in verifying or disproving these propositions. In principle, extensive experimental measurements of correlated energy distributions should permit one to confirm or reject the present interpretation of a statistical dissociation with an exit barrier. However, since we have observed only a limited fraction of the fragment quantum states, we must avoid drawing sweeping conclusions about the global dynamics based on our results alone.

A calculation by North and Gezelter $[120,121]$ suggests that such caution is reasonable, and also points to an explanation for the similar translational energy distributions of both methyl vibrational states. The objective of their general approach is to describe statistical dissociations with an exit barrier. They assume that energy in excess of the dissociation barrier is partitioned statistically, while the remaining fraction is released impulsively by the exit barrier. Furthermore, for azomethane, the first methyl is lost with no exit barrier and only the second departs impulsively. Using this model, the average vibrational energy appearing in the first and second methyls was calculated to be $8.2 \mathrm{kcal} / \mathrm{mol}$ and $9.9 \mathrm{kcal} / \mathrm{mol}$, respectively, with both values dominated by the initial statistical partitioning. Most of the methyl radicals would 
therefore be found in vibrational states other than the two which we have measured (one quantum of $\nu_{2}$ is only $606 \mathrm{~cm}^{-1}$ or $1.73 \mathrm{kcal} / \mathrm{mol}$ ), and it would not be surprising for this limited subset to have translational energy distributions much different from the average measured by North et al. Furthermore, whether a methyl has zero or one quantum of umbrella vibration may matter less than the fact that both types are near the same extreme with respect to average vibrational energy. On the other hand, if a proposed mechanism predicted low average vibrational energies, then our distributions should be more representative of the average, and thus more difficult to reconcile with that of North.

In fact, the basic arguments above do not depend on the details of North and Gezelter's model-in general, statistical partitioning of a large excess energy will leave substantial vibrational energy in most of the methyl fragments. In contrast, methyls which depart prior to energy randomization would contain far less vibrational energy on average. Even an impulsive dissociation over an exit barrier is not expected to impart significant methyl vibrational energy. Amatatsu et al. have explained the latter point very clearly in relation to their classical trajectory simulations for methyl iodide dissociation [122]. They show that efficient umbrella excitation occurs when the methyl umbrella potential evolves rapidly compared to the separation of the fragments. For the ground state channel, there is a sharp discontinuity in the shape of the umbrella potential at a curve crossing, which leads to an inverted vibrational distribution. In contrast, the $I^{*}$ channel experiences only the smooth opening of the umbrella potential, on a timescale comparable to the vibrational frequency. The corresponding vibrational distribution is peaked at $v_{2}=0$.

Based on these general trends we can suggest three possibilities for the origin of the low-vibrating methyls which we have detected. Even in a largely statistical dissociation, some fraction of the molecules may dissociate prior to randomization, and the resulting fragments will contain less vibrational energy than average. Moreover, these fragments can surmount the dissociation barrier with much of the balance of energy in the dissociation coordinate, leading to a large fraction released into translation. Thus even the primary methyl in azomethane, which is not repelled by an exit barrier and would otherwise come off slowly, would also have substantial kinetic 
energy. While these characteristics describe our observations quite well, molecules which dissociate under these conditions are likely to represent only a small subset of the total excited population. Longer-surviving parent molecules would also populate the low methyl vibrations, possibly swamping the former effect. For such a mechanism to apply, there would have to be an efficient non-statistical detour near the internal conversion geometry, which would allow a significant fraction of trajectories to escape before energy scrambling.

A less exotic possibility is that the dissociation is truly prompt, and most of the parent molecules dissociate rapidly. Although Weisman et al. speculated that the 'small slopes' of the methyldiazenyl exit barrier could produce a statistical-like translational energy release in that step, such behavior would be unprecedented. Instead, we would expect both methyl photofragments to be fast and contain little vibrational energy, as seen in our measurements. However, a mechanism which produces two fast methyls is not consistent with the total translational energy distribution of North et $a l .$, nor with the theoretical predictions of Liu et al. In order to more confidently exclude this mechanism from consideration, we should determine whether the apparent shift with wavelength in the high-energy tail of our translational energy distributions is real or an experimental artifact.

If we assume only that statistical randomization occurs to a large extent, the shape of the translational energy distributions suggests localized energy conservation for the methyl radical -i.e. the low energy wing of the vibrational energy distribution correlates to the high energy wing of the translational energy distribution. Of course, this explanation begs the question of why such a correlation should exist and demands verification from a detailed simulation of statistical energy partitioning. One possibility is that minimal vibrational energy in the still-bound secondary methyl leaves more energy available to surmount the barrier to loss of the primary (slower) methyl. In any case, a purely statistical picture is likely to be an oversimplification, and one should also consider the role of dynamical effects caused by the exit barrier. According to the prevailing interpretation of a concerted dissociation, only the second methyl is exposed to the exit barrier, corresponding to the high translational energy component in our measurements. The exit barrier may deexcite a fraction of the 
otherwise-excited secondary methyls, thus increasing the correlation between the low vibrational states and faster translation. That such effects are stronger for the steep repulsive potential of the secondary methyl is generally consistent with the classical trajectory calculations of Amatatsu et al. [122], although even then, the effect would probably be very weak.

\subsection{Conclusion}

The ultraviolet dissociation of azomethane has not yet been fully tapped as a rich source of insight into three-fragment dissociation dynamics. The vibrationcorrelated translational energy distributions for methyl photofragments reported here have opened a small but revealing window into the finer details of azomethane dissociation. The invariance of the distributions with vibrational state and the preference for faster fragments are both consistent with the previously-proposed mechanism of energy scrambling followed by concerted dissociation. However, one cannot rule out alternatives such as rapid impulsive dissociation based on these data alone. Classical trajectory simulations should permit a more concrete assignment of the detailed processes responsible for producing this small subset of fragment quantum states. The inherent complexity of the three-body process is further complicated by the experimental challenge of a low absorption cross section at long wavelengths and relatively efficient multiphoton absorption. Given these difficulties, it will not be a small task to measure a wider range of fragment quantum states using conventional experimental techniques, however the results should be very rewarding. 


\section{References}

[1] V. Bush, Science: The Endless Frontier. A report to the President by Vannevar Bush, Director of the Office of Scientific Research and Development, July 1945, National Science Foundation, Washington, D.C., 1990.

[2] R.B. Bernstein, D.R. Herschbach, and R.D. Levine, Dynamical aspects of stereochemistry, J. Phys. Chem. 91, 5365 (1987).

[3] J.P. Simons, Dynamical stereochemistry and the polarization of reaction products, J. Phys. Chem 91, 5378 (1987).

[4] Orientation and polarization effects in chemical reaction dynamics, J. Chem. Soc. Faraday Trans. 89 (1993).

[5] J. Solomon, Photodissociation as studied by photolysis mapping, J. Chem. Phys. 47, 889 (1967).

[6] R.N. Zare and D.R. Herschbach, Doppler line shape of atomic fluorescence excited by molecular photodissociation, Proc. IEEE 51, 173 (1963).

[7] R.N. Zare, Photoejection dynamics, Molecular Photochemistry 4, 1 (1972).

[8] C. Jonah, Effect of rotation and thermal velocity on the anisotropy in photodissociation spectroscopy, J. Chem. Phys. 55, 1915 (1971).

[9] S. Yang and R. Bersohn, Theory of the angular distribution of molecular photofragments, J. Chem. Phys. 61, 4400 (1974). 
[10] G.E. Busch, R.T. Mahoney, R.I. Morse, and K.R. Wilson, Translational spectroscopy of $\mathrm{Cl}_{2}$, J. Chem. Phys. 51, 449 (1969).

[11] R.W. Diesen, J.C. Wahr, and S.E. Adler, Photochemical recoil spectroscopy, J. Chem. Phys. 50, 3635 (1969).

[12] D. Bassi, Ionization methods, in G. Scoles, editor, Atomic and Molecular Beams Methods, chapter 7, p. 168, Oxford University Press, New York, 1988.

[13] L. Schnieder, W. Meier, K.H. Welge, M.N.R. Ashfold, and C.M. Western, Photodissociation dynamics of $\mathrm{H}_{2} \mathrm{~S}$ at $121.6 \mathrm{~nm}$ and a determination of the potential energy function of $S H\left(A^{2} \Sigma^{+}\right)$, J. Chem. Phys. 92, 7027 (1990).

[14] R.E. Continetti, D.R. Cyr, D.L. Osborn, D.J. Leahy, and D.M. Neumark, Photodissociation of the $N_{3}$ radical, J. Chem. Phys. 99, 2616 (1993).

[15] D.P de Bruijn and J. Los, Time and position-sensitive detector for dissociative processes in fast beams, Rev. Sci. Instrum. 53, 1642 (1982).

[16] J.A. Syage, Photofragment imaging by sections for measuring state-resolved angle-velocity differential cross sections, J. Chem. Phys. 105, 1007 (1996).

[17] L.H. Lai, D.C. Che, and K. Liu., Photodissociative pathways of $\mathrm{C}_{2} \mathrm{H}_{2}$ at 121.6 $n m$ revealed by a Doppler-selected time-of-fight (a 3-D imaging) technique.

[18] A. Sanov, C.R. Bieler, and H.Reisler, Fully quantum-state resolved study of $\mathrm{NO}_{2}$ photodissociation: Correlated $N O\left({ }^{2} \Pi_{\Omega}, v=0, j, \Lambda\right)+O\left({ }^{3} P_{j}\right)$ distributions, J. Phys. Chem. 99, 13637 (1995).

[19] H. Ni, J.M. Serafin, and J.J. Valentini, State-resolved three-dimensional product recoil velocity spectroscopy, J. Chem. Phys. 104, 2259 (1996).

[20] K. Tonokura and T. Suzuki, Slicing photofragment spatial distribution by laser sheet ionization, Chem. Phys. Lett. 224, 1 (1994).

[21] R.J. Gordon and G.E. Hall, Applications of Doppler spectroscopy in photofragmentation, Adv. Chem. Phys. (1996). 
[22] S.W. North, X.S. Zheng, R. Fei, and G.E. Hall, Line shape analysis of Dopplerbroadened frequency-modulated line spectra, J. Chem. Phys. 104, 2129 (1996).

[23] A.J. Orr-Ewing and R.N. Zare, Orientation and alignment of reaction products, Annu. Rev. Phys. Chem. 45, 315 (1994).

[24] G.E. Hall and P.L. Houston, Vector correlations in photodissociation dynamics, Annu. Rev. Phys. Chem. 40, 375 (1989).

[25] P.L. Houston, Vector correlations in photodissociation dynamics, J. Phys. Chem. 91, 5388 (1987).

[26] U. Fano and G. Racah, Irreducible Tensorial Sets, Academic, New York, 1959.

[27] U. Fano and J.H. Macek, Impact excitation and polarization of emitted light, Rev. Mod. Phys. 45, 553 (1973).

[28] D.R. Herschbach D.A. Case, G.M. McClelland, Angular momentum polarization in molecular collisions: Classical and quantum theory for measurements using resonance fluorescence, Mol. Phys. 35, 541 (1978).

[29] C.H. Greene and R.N. Zare, Photofragment orientation and alignment, Annu. Rev. Phys. Chem. 33, 119 (1982).

[30] C.H. Greene and R.N. Zare, Determination of product population and alignment using laser-induced fluorescence., J. Chem. Phys. 78, 6741 (1983).

[31] G.E. Hall, N. Sivakumar, P.L. Houston, and I. Burak, Measurement of the angular correlation between recoil velocity and angular momentum vectors in molecular photodissociation, Phys. Rev. Lett. 56, 1671 (1986).

[32] M. Dubs, U. Brühlmann, and J. R. Huber, Sub-Doppler laser-induced fluorescence measurements of the velocity distribution and rotational alignment of $N O$ photofragments, J. Chem. Phys. 84, 3106 (1986). 
[33] K.H. Gericke, S. Klee, F.J. Comes, and R.N. Dixon, Dynamics of $\mathrm{H}_{2} \mathrm{O}_{2}$ photodissociation: $\mathrm{OH}$ product state and momentum distribution characterized by sub-Doppler and polarization spectroscopy, J. Chem. Phys. 85, 4463 (1986).

[34] M.P. Docker, A. Hodgson, and J.P. Simons, Photodissociation of $\mathrm{H}_{2} \mathrm{O}_{2}$ at 248 $n m$ : translational anisotropy and $O H$ product state distributions, Chem. Phys. Lett. 128, 264 (1986).

[35] R.J. Van Brunt and R.N. Zare, Polarization of atomic fluorescence excited by molecular dissociation, J. Chem. Phys. 48, 4304 (1968).

[36] M.T. MacPherson, J.P. Simons, and R.N. Zare, Polarized photofluorescence excitation spectroscopy. Calculation of the degree of polarization of the fluorescence from diatomic molecular fragments produced through photodissociation of triatomic molecules, Mol. Phys. 38, 2049 (1979).

[37] L.C. Biedenharn, Angular correlations in nuclear spectroscopy, in F. AjzenbergSelove, editor, Nuclear Spectroscopy, p. 732, Academic Press, New York, 1960.

[38] R.N. Dixon, The determination of the vector correlation between photofragment rotational and translational motions from the analysis of Doppler-broadened spectral line profiles, J. Chem. Phys. 85, 1866 (1986).

[39] G.E. Hall, N. Sivakumar, D. Chawla, P.L. Houston, and I. Burak, Angular correlations between recoil velocity and angular momentum vectors in molecular photodissociation, J. Chem. Phys. 88, 3682 (1988).

[40] K.M. Chen, D.J. Tanno, and D.G. Imre, Determination of $k^{\prime}-J^{\prime}$ correlations of reaction products by fluorescence-imaging techniques. 1. linearly polarized excitation, J. Phys. Chem. 101, 2525 (1997).

[41] L.D.A Siebbeles, M. Glass-Maujean, O.S. Vasyutinskii, J.A. Beswick, and O. Roncero, Vector properties in photodissociation: Quantum treatment of the correlation between the spatial anisotropy and the angular momentum polarization of the fragments, J. Chem. Phys. 100, 3610 (1994). 
[42] O.S. Vasyutinskii, Orientation of atoms during photodissociation of molecules, Sov. Phys. JETP Lett. 31, 428 (1980).

[43] E.W. Rothe, U. Krause, and R. Duren, Observation of polarization of atomic fluorescence excited by laser-induced dissociation of $\mathrm{Na}_{2}$, Chem. Phys. Lett. 72, $100(1980)$.

[44] A.C.G. Mitchell, Über die Richtungsverteilung der Relativgeschwindigkeit der Zerfallsprodukte bei optischer Dissoziation von Jodnatrium, Z. Phys. 49, 228 (1928).

[45] D.V. Kupriayanov and O.S. Vasyutinskii, Orientation and alignment of ${ }^{2} P_{3 / 2}$ fragments following photodissociation of heteroatomic molecules, Chem. Phys. 171, 25 (1993).

[46] O.S. Vasyutinskii and J.A. Beswick, Long-range interatomic interactions studied through polarized photofragments techniques, Comm. At. Mol. Phys., in press (1997).

[47] J. Vigué, J.A. Beswick, and M. Broyer, Coherence effects in the polarization of the light emitted by photofragments, J. Physique 44, 1225 (1983).

[48] O.S. Vasyutinskii, Orientation and alignment of atoms in photodissociation of molecules with consideration of atomic motion, Khim. Fiz. 5, 768 (1986).

[49] M. Glass-Maujean and J.A. Beswick, New coherence effects in the polarization of photofragments: Theory, Phys. Rev. A 36, 1170 (1987).

[50] M. Glass-Maujean and J.A. Beswick, Coherence effects in the polarization of photofragments, J. Chem. Soc. Faraday Trans. 85, 983 (1989).

[51] S.J. Singer, K.F. Freed, and Y.B. Band, Theory of diatomic molecule photodissociation: Electronic angular momentum influence on fragment and fluorescence cross sections, J. Chem. Phys 79, 6060 (1983). 
[52] A.G. Suits and P.L. Houston, Alignment effects and imaging: boon or bane?, in Workshop on Imaging Methods in Molecular Structure and Dynamics, Neve Ilan, Israel, 1994.

[53] T. Suzuki, H. Katayanagi, Y. Mo, and K. Tonokura, Evidence for multiple dissociation components and orbital alignment in $205 \mathrm{~nm}$ photodissociation of $\mathrm{N}_{2} \mathrm{O}$, Chem. Phys. Lett. 256, 90 (1996).

[54] Y. Mo, H. Katayanagi, M.C. Heaven, and T. Suzuki, Simultaneous measurement of recoil velocity and alignment of $S\left({ }^{1} D_{2}\right)$ atoms in photodissociation of OCS, Phys. Rev. Lett. 77, 830 (1996).

[55] Y. Wang, H.P. Loock, J. Cao, and C.X.W. Qian, Atomic photofragment $v-j$ correlation: Dissociation of $\mathrm{Cl}_{2}$ at $355 \mathrm{~nm}$, J. Chem. Phys. 102, 808 (1995).

[56] A.S. Bracker, E.R. Wouters, A.G. Suits, Y.T. Lee, and O.S. Vasyutinskii, Observation of coherent and incoherent dissociation mechanisms in the angular distribution of atomic photofragment alignment, Phys. Rev. Lett., in press (1997).

[57] T.P. Rakitzis and R.N. Zare, 1997, Private communication.

[58] D.W. Chandler and P.L. Houston, Two-dimensional imaging of state-selected photodissociation products detected by multiphoton ionization, J. Chem. Phys. 87, 1445 (1987).

[59] R.N. Strickland and D.W. Chandler, Reconstruction of an axisymmetric image from its blurred and noisy projection, Appl. Opt. 30 (1991).

[60] B.J. Whitaker, Photo-ion imaging and future directions in reactive scattering, in R.G. Compton and G. Hancock, editors, Research in chemical kinetics, volume 1, p. 307, Elsevier, Amsterdam, 1994.

[61] P.L. Houston, New laser-based and imaging methods for studying the dynamics of molecular collisions, J. Phys. Chem. 100, 12757 (1996). 
[62] A.J.R. Heck and D.W. Chandler, Imaging techniques for the study of chemical reaction dynamics, Annu. Rev. Phys. Chem. 46, 335 (1995).

[63] D. Proch and T. Trickl, A high-intensity multi-purpose piezoelectric pulsed molecular beam source, Rev. Sci. Inst. 60, 713 (1989).

[64] E.F. Cromwell, Dynamics of $\mathrm{H}_{2}$ Elimination from Unsaturated Hydrocarbons, $\mathrm{PhD}$ thesis, University of California, Berkeley, 1991.

[65] S. Arepalli, N. Presser, D. Robie, and R.J. Gordon, Detection of $\mathrm{Cl}$ atoms and $\mathrm{HCl}$ molecules by resonantly-enhanced multiphoton ionization, Chem. Phys. Lett. 118, 88 (1985).

[66] S.T. Pratt, P.M. Dehmer, and J.L. Dehmer, Double-resonance spectroscopy of transitions between autoionizing levels of atomic oxygen, Phys. Rev. A 43, 4702 (1991).

[67] K. Omidvar, Two-photon excitation cross section in light and intermediate atoms in frozen-core LS-coupling approximation, Phys. Rev. A 22, 1576 (1980).

[68] K. Omidvar, Erratum: Two-photon excitation cross section in light and intermediate atoms in frozen-core LS-coupling approximation, Phys. Rev. A 30, 2805 (1984).

[69] D.H. Parker and A.T.J.B. Eppink, Photoelectron and photofragment velocity map imaging of state-selected molecular oxygen dissociation/ionization dynamics, J. Chem. Phys. 107, 2357 (1997).

[70] A.T.J.B. Eppink and D.H. Parker, Velocity map imaging of ions and electrons using electrostatic lenses: Application in photoelectron and photofragment ion imaging of molecular oxygen, Rev. Sci. Instr. 68, 3477 (1997).

[71] W.C. Wiley and I.H. McLaren, Time-of-flight mass spectrometer with improved resolution, Rev. Sci. Inst. 26, 1150 (1955). 
[72] Z. Xu, B. Koplitz, and C. Wittig, Velocity-aligned Doppler spectroscopy, J. Chem. Phys. 90, 2692 (1989).

[73] R.N. Zare, Angular Momentum, World Scientific, New York, 1988.

[74] K. Blum, Density Matrix Theory and Applications, Plenum Press, New York, 1989.

[75] R.D. Hinchliffe R. Uberna and J.I. Cline, Photofragment $\mu-v-j$ correlation measured by $1+n^{\prime}$ resonance-enhanced multiphoton ionization: Selective probing of bipolar moments and detection of chiral dynamics, J. Chem. Phys. 103, 7934 (1995).

[76] S.W. North and G.E. Hall, Quantum phase space theory for the calculation of $v-j$ vector correlations, J. Chem. Phys. 104, 1864 (1996).

[77] B.V. Picheyev, A.G. Smolin, and O.S. Vasyutinskii, Ground state polarized photofragments study by using resonance and off-resonance probe beam techniques, J. Phys. Chem. 101, 7614 (1997).

[78] A.C. Kummel, G.O. Sitz, and R.N. Zare, Determination of population and alignment of the ground state using two-photon nonresonant excitation, J. Chem. Phys. 85, 6874 (1986).

[79] A.C. Kummel, G.O. Sitz, and R.N. Zare, Determination of orientation of the ground state using two-photon nonresonant excitation, J. Chem. Phys. 88, 6707 (1988).

[80] C.J. Smith, E.M. Spain, M.J. Dalberth, S.R. Leone, and J.P.J. Driessen, Initial and final orbital alignment probing of the fine-structure- changing collisions among $C a(4 s)^{1}(4 p)^{1}{ }^{3} P_{J}$ states with he: Determination of coherence and conventional cross-sections, J. Chem. Soc. Faraday Trans. 89, 1401 (1993).

[81] A.G. Suits, H.T. Hou, H.F. Davis, and Y.T. Lee, Reaction geometry from orbital alignment dependence of ion pair production in crossed beams $B a\left({ }^{1} P_{1}\right)$ $\mathrm{Br}_{2}$ reactions, J. Chem. Phys. 95, 8178 (1991). 
[82] D. Maric, J.P. Burrows, R. Meller, and G.K. Moorgat, A study of the uv-visible absorption spectrum of molecular chlorine, J. Photochem. Photobiol. A 70, 205 (1993).

[83] R.S. Mulliken, Correlation of atomic $J$ values and molecular quantum numbers, with applications to halogen, alkaline earth hydride, and alkali molecules, Phys. Rev. 36, 1440 (1930).

[84] G. Herzberg, Molecular Spectra and Molecular Structure I, Spectra of Diatomic Molecules, Van Nostrand Reinhold, New York, 1950.

[85] Y. Matsumi, K. Tonokura, and M. Kawasaki, Fine-structure branching ratios and Doppler profiles of $\mathrm{Cl}\left({ }^{2} P_{j}\right)$ photofragments from photodissociation of the chlorine molecule near and in the ultraviolet region, J. Chem. Phys. 97, 1065 (1992).

[86] L. Li, R.J. Lipert, J. Lobue, W.A. Chupka, and S.D. Wilson, Adiabatic dissociation of photoexcited chlorine molecules, Chem. Phys. Lett. 151, 335 (1988).

[87] Y. Matsumi, M. Kawasaki, T. Sato, T Kinugawa, and T. Arikawa, Photodissociation of chlorine molecule in the UV region, Chem. Phys. Lett. 97, 486 (1992).

[88] P.C. Samartzis, I. Sakellariou, T. Gougousi, and T.N. Kitsopoulos, Photofragmentation study of $\mathrm{Cl}_{2}$ using ion imaging, J. Chem. Phys. 107, 43 (1997).

[89] Y.B. Band, K.F. Freed, and D.J. Kouri, Dissociation of diatomic molecules into atoms with non-vanishing electronic angular momentum. Physical motivation for the theory, Chem. Phys. Lett. 79, 233 (1981).

[90] Y.B. Band and K.F. Freed, Dissociation of diatomic molecules into atoms with non-vanishing electronic angular momentum. Theory, Chem. Phys. Lett. 79, 238 (1981). 
[91] A.J. Orr-Ewing, W.R. Simpson, T.P. Rakitzis, S.A. Kandel, and R.N. Zare, Scattering-angle resolved product rotational alignment for the reaction of $\mathrm{Cl}$ with vibrationally excited methane, J. Chem. Phys. 106, 5961 (1997).

[92] J. Vigué, P. Grangier, G. Roger, and A. Aspect, Polarization of calcium atomic fluorescence due to a coherence effect in the photodissociation of $\mathrm{Ca}_{2}$ molecules, J. Physique Lett. 42 (1981).

[93] E.E. Nikitin and S.Y. Umanskii, Theory of Slow Atomic Collisions, SpringerVerlag, Berlin, 1984.

[94] O.S. Vasyutinskii, Contribution to the theory of the effect of orientation of atoms produced in photodissociation of molecules, Zh. Eksp. Teor. Fiz. 81, 855 (1981).

[95] D.A. Varshalovich, A.N. Moskalev, and V.K. Khersonskii, Quantum Theory of Angular Momentum, World Scientific, Singapore, 1988.

[96] T. Ishiwata, A. Ishiguro, and K. Obi, Optical-optical double resonance spectroscopy of $\mathrm{Cl}_{2}$ : Analysis of the $A^{3} \Pi\left(1_{u}\right)-X^{1} \Sigma_{g}^{+}$system, J. Molec. Spec. 147, 300 (1991).

[97] C.E. Fairchild, E.J. Stone, and G.M. Lawrence, Photofragment spectroscopy of ozone in the UV region 270-310 $\mathrm{nm}$ and at $600 \mathrm{~nm}$, J. Chem. Phys. 69, 3632 (1978).

[98] R.K. Sparks, L.R. Carlson, K. Shobatake, M.L. Kowalczyk, and Y.T. Lee, Ozone photolysis: A determination of the electronic and vibrational state distributions of primary products, J. Chem. Phys. 72, 1401 (1979).

[99] D. Stranges, X. Yang, J.D. Chesko, and A.G. Suits, Photodissociation of ozone at $193 \mathrm{~nm}$ by high-resolution photofragment translational spectroscopy, J. Chem. Phys. 102, 6067 (1995). 
[100] J.J. Valentini, D.P. Gerrity, D.L. Philliips, J.C. Nieh, and K.D. Tabor, CARS spectroscopy of $\mathrm{O}_{2}\left({ }^{1} \Delta_{g}\right)$ from the Hartley band photodissociation of $\mathrm{O}_{3}$ : Dynamics of the dissociation, J. Chem. Phys. 86, 6745 (1987).

[101] T. Kinugawa, T. Sato, T. Arikawa, Y. Matsumi, and M. Kawasaki, Formation of $O\left({ }^{3} P_{j}\right)$ photofragments from the Hartley band photodissociation of ozone at 226 nm, J. Chem. Phys. 93, 3289 (1990).

[102] A.G. Suits, R.L. Miller, L.S. Bontuyan, and P.L. Houston, Photofragment vector correlations by ion imaging: $\mathrm{O}_{2}\left[a^{1} \Delta_{g}(v, J)\right]$, J. Chem. Soc. Faraday Trans. 89, 1443 (1993).

[103] D. Chasman, D.J. Tanno, and D.G. Imre, Photoabsorption and photoemission of ozone in the Hartley band, J. Chem. Phys. 89, 6667 (1988).

[104] B.R. Johnson and J.L. Kinsey, Time-dependent analysis of the Hartley absorption band and resonance Raman spectra in ozone, J. Chem. Phys. 87, 1525 (1987).

[105] A. Banichevich, S.D. Peyerimhoff, and F. Grein, Potential energy surfaces of ozone in its ground state and in the lowest-lying eight excited states, Chem. Phys. 178, 155 (1993).

[106] A. Banichevich and S.D. Peyerimhoff, Theoretical study of the ground and excited states of ozone in its symmetric nuclear arrangement, Chem. Phys. 174, 93 (1993).

[107] H.C. Ramsperger, The decomposition of azomethane. A homogenous unimolecular reaction, J. Am. Chem. Soc. 49, 912 (1927).

[108] P.S. Engel, Mechanism of the thermal decomposition of azoalkanes, Chem. Rev. 80, 99 (1980).

[109] R. Liu, Q. Cui, K.M. Dunn, and K. Morokuma, Ab initio molecular orbital study of the mechanism of photodissociation of trans-azomethane, J. Chem. Phys. 105, 2333 (1996). 
[110] G. Kortum and H. Rau, Spektroskopische untersuchungen an der azogruppe, Ber. Buns. 68, 973 (1964).

[111] M.B. Robin, R.R. Hart, and N.A. Kuebler, Electronic states of the azoalkanes, J. Am. Chem. Soc. 89, 1564 (1967).

[112] O.A. Mosher, M.S. Foster, W.M. Flicker, J.L. Beauchamp, and A. Kuppermann, Electronic spectroscopy of trans-azomethane by electron impact, J. Chem. Phys. 62, 3424 (1975).

[113] K.A. Burton and R.B. Weisman, Stepwise photodissociation of vapor-phase azomethane, J. Am. Chem. Soc. 112, 1804 (1990).

[114] B.K. Andrews, K.A. Burton, and R.B. Weisman, Dynamics of the two-step photodissociation of azomethane, J. Chem. Phys. 96, 1111 (1992).

[115] S.W. North, C.A. Longfellow, and Y.T. Lee, The near ultraviolet photodissociation dynamics of azomethane, J. Chem. Phys. 99, 4423 (1993).

[116] D.H. Fairbrother, K.A. Dickens, P.C. Stair, and E. Weitz, Energy content of methyl radicals produced in the UV photodissociation of azomethane, Chem. Phys. Lett. 246, 513 (1995).

[117] J.W. Hudgens, T.G. DiGiuseppe, and M.C. Lin, Two photon resonance enhanced multiphoton ionization spectroscopy and state assignments of the methyl radical, J. Chem. Phys. 79, 571 (1983).

[118] W.R. Simpson, A.J. Orr-Ewing, T.P. Rakitzis, S.A. Kandel, and R.N. Zare, Core extraction for measuring state-to-state differential cross sections in bimolecular reactions, J. Chem. Phys. 103, 7299 (1995).

[119] B.K. Andrews and R.B. Weisman, Structure and dissociation of the methyldiazenyl radical: A quadratic configuration interaction computational study, J. Chem. Phys. 101, 6776 (1994). 
[120] S.W. North, Photofragment Translational Spectroscopy of Three Body Dissociations and Free Radicals, $\mathrm{PhD}$ thesis, University of California, Berkeley, 1995.

[121] S.W. North, D.A. Blank, C.A. Longfellow, J.D. Gezelter, C.A. Longfellow, and Y.T. Lee, Evidence for stepwise dissociation dynamics in acetone at 248 and 193 nm., J. Chem. Phys. 102, 4447 (1995).

[122] Y. Amatatsu, K. Morokuma, and S. Yabushita, Ab initio potential energy surfaces and trajectory studies of A-band photodissociation dynamics: $\mathrm{CH}_{3} \mathrm{I}^{*} \rightarrow$ $\mathrm{CH}_{3}+\mathrm{I}$ and $\mathrm{CH}_{3}+\mathrm{I}^{*}$, J. Chem. Phys. 94, 4858 (1991). 
M98052893

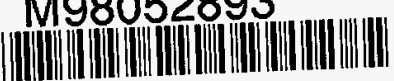

Report Number (14) LBNL- $4 / 402$

subl. Date (11) $\quad / 997 / 2$

Sponsor Code (18) DOE/ER;NSF, XF

JC Category (19) UC-40i; UC-000, DOE/ER

DOE 